Supporting Information for

\title{
Few-Layered Metal-Organic Framework Nanosheets as Catalysts for the Synthsis of 2,3-Dihydroquinazolinone and Propargylamines
}

\author{
Qingchun Xia, ${ }^{*}$, Suzhen Zhang, ${ }^{\dagger}$ Yingxin Zhang,${ }^{\dagger}$ Ruixiang Bai, ${ }^{\dagger}$ Shujun Li, ${ }^{\dagger}$ Jie Zhang,,${ }^{, \dagger}$ and \\ Xuenian Chen*,广,+ \\ ${ }^{\dagger}$ Henan Key Laboratory of Boron Chemistry and Advanced Energy Materials, Collaborative Innovation Center of \\ Henan Province for Green Manufacturing of Fine Chemicals, Key Laboratory of Green Chemical Media and Reac- \\ tions, Ministry of Education, School of Chemistry and Chemical Engineering, Henan Normal University, Xinxiang, \\ Henan 453007, China. \\ * Green Catalysis Center and College of Chemistry, Zhengzhou University, Zhengzhou, Henan 450001, China.
}

Email: xiaqingchun@htu.edu.cn; Jie.zhang@htu.edu.cn; Xuenian_Chen@zzu.edu.cn

\section{Table of Content}

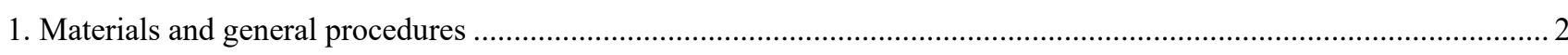

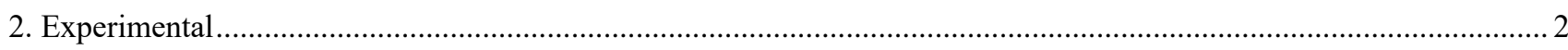

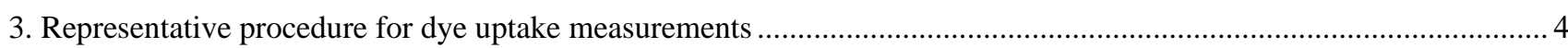

4. Table S1. Crystal data and structure refinement for LMOF 1. ............................................................................ 5

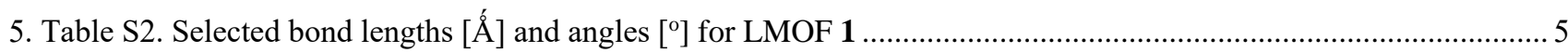

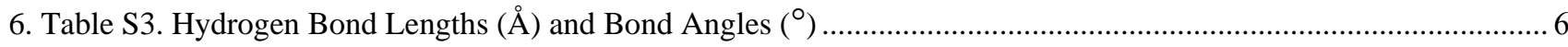

7. Figure S1 S4. Additional X-ray crystallographic structures .................................................................................. 6

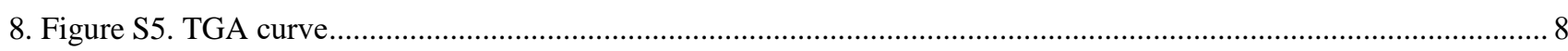

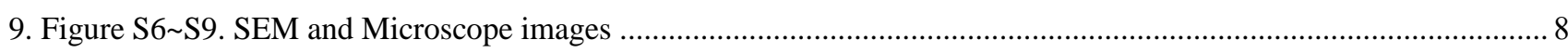

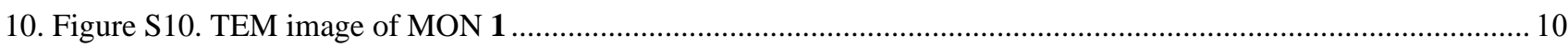

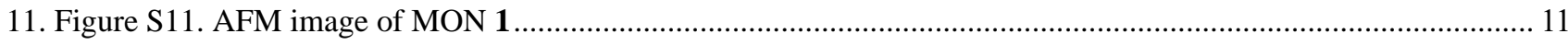

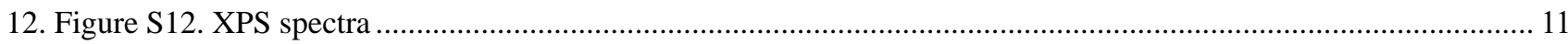

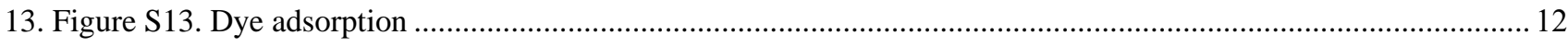

14. Figure S14. The plausible mechanism for the formation of 2,3-dihydroquinazolinone.............................................. 13

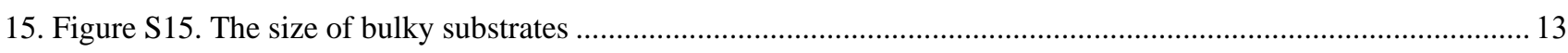

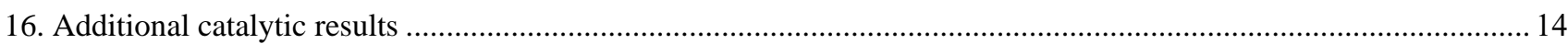

17. Table S6. Summary of the solvents used for ultrasonic exfoliation........................................................................ 16

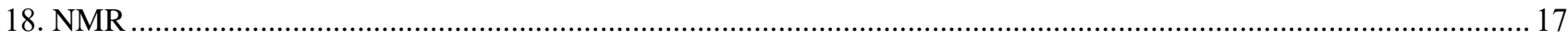

19. Figure S16. FT-IR spectra of LMOF 1 and MON 1 after 5 catalytic runs ............................................................... 34 


\section{Materials and general procedures}

All reagents and solvents used in these studies are commercially available and used without further purification. Elemental analyses were performed with an EA1110 CHNS-0 CE elemental analyzer. The IR (KBr pellet) spectra were recorded (400-4000 $\mathrm{cm}^{-1}$ region) on a Nicolet Magna $750 \mathrm{FT}$ IR spectrometer. Thermogravimetric analyses (TGA) were carried out in an air atmosphere with a heating rate of $10{ }^{\circ} \mathrm{C} / \mathrm{min}$ on a STA449C integration thermal analyzer. Powder X-ray diffraction (PXRD) data were collected on a DMAX2500 diffractometer using $\mathrm{Cu}-\mathrm{K} \alpha$ radiation. The calculated PXRD patterns were produced using the SHELXTL-XPOW program and single crystal reflection data. The NMR experiments were carried out on a MERCURY plus 400 spectrometer operating at resonance frequencies of $400 \mathrm{MHz}$. Electrospray ionization mass spectra (ESI-MS) were recorded on a Finnigan LCQ mass spectrometer using dichloromethane-methanol as mobile phase. ICP-OES was performed on Optima 7300DV ICP-OES (Perkin Elmer Coporation, USA). The $\mathrm{CO}_{2}$ adsorption isotherms were recorded at $273 \mathrm{~K}$ by using a micromeritics ASAP 2020 surface area and porosity analyzer. Before the adsorption measurement, the samples were activated at $80^{\circ} \mathrm{C}$ under vacuum $\left(<10^{-3}\right.$ torr $)$ for $4 \mathrm{~h}$. Scanning Electron Microscopy (SEM) images were performed on a NOVA NanoSEM 230 instrument equipped with an energy dispersive spectroscopy (EDS) detector. X-ray photoelectron spectroscopy (XPS) were recorded on a AXIS Ultra DLD surface analysis instrument.

Single-Crystal X-ray Diffraction. Single-crystal XRD data for LMOF 1 was collected on a Bruker SMART Apex II CCD-based X-ray diffractometer with $\mathrm{Cu}-\mathrm{K} \alpha$ radiation $(\lambda=1.54178 \AA$ ) at $150 \mathrm{~K}$. We have collected about several datasets for LMOF 1 using $\mathrm{Cu}-\mathrm{K} \alpha$ radiation. Among the several datasets, the best dataset was used for structure solution and refinement. The empirical absorption correction was applied by using the SADABS program (G. M. Sheldrick, SADABS, program for empirical absorption correction of area detector data; University of Göttingen, Göttingen, Germany, 1996). The structure was solved by direct methods with SHELXS-2018 and refined with SHELXL-2018 using OLEX 1.2. In the structure, all the non-hydrogen atoms were refined by full-matrix least-squares techniques with anisotropic displacement parameters, and the hydrogen atoms were geometrically fixed at the calculated positions attached to their parent atoms, treated as riding atoms. Contributions to scattering due to these highly disordered solvent molecules except two DMF molecules were removed using the SQUEEZE routine of PLATON; structures were then refined again using the data generated.

The structure was then refined again using the data generated. Crystal data and details of the data collection are given in Table S1, while the selected bond distances and angles are presented in Table S2. CCDC 2087493 contains the supplementary crystallographic data for this paper. These data can be obtained free of charge from the Cambridge Crystallographic Data Centre via www.ccdc.cam.ac.uk/data request/cif

\section{Experimental}


Prior to catalysis, the MOFs and MONs were exchanged with chloroform for two days and then activated under vacuum at room temperature for $4 \mathrm{~h}$ to remove the guest solvents.

\subsection{Condensation and cyclization of 2-aminobenzamide with aldehydes to synthesize 2,3- dihydroquinazolinone}

To a flame-dried schlenk pressure tube was added activated catalyst $\left(5.0 \mathrm{~mol} \%\right.$, based on the $\left.\mathrm{Cu}^{\mathrm{II}}\right)$, 2-aminobenzamide $(13.6 \mathrm{mg}, 0.1 \mathrm{mmol})$, aldehyde $(0.11 \mathrm{mmol})$ and $\mathrm{MeOH}(1 \mathrm{~mL})$. The mixture was stirred at $70{ }^{\circ} \mathrm{C}$ under nitrogen for $12 \mathrm{~h}$. After that, the mixture was centrifuged at $9000 \mathrm{rpm}$ for $5 \mathrm{~min}$, the supernatant was concentrated under vacuum and then purified by column chromatography on silica gel (EtOAc/ petroleum ether, 1/2, v/v) to afford the desired products.

The conversion was calculated according to ${ }^{1} \mathrm{H}$ NMR of the reaction solutions. The mixture was centrifuged to remove the MOF, and then the supernatant was concentrated under vacuum to remove the solvent, the residue was dissolved in $\mathrm{CDCl}_{3}$ or DMSO- $d_{6}$ to get the crude ${ }^{1} \mathrm{H} \mathrm{NMR}$.

For example:

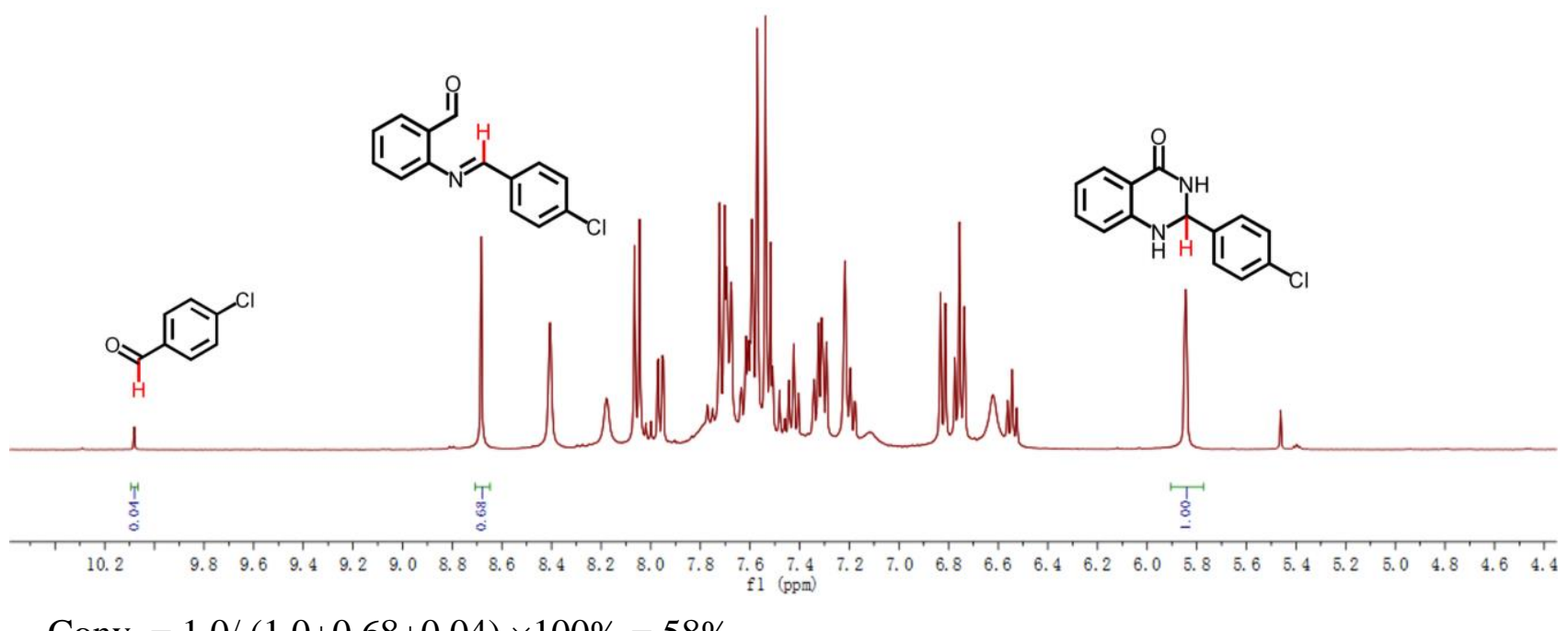

Conv. $=1.0 /(1.0+0.68+0.04) \times 100 \%=58 \%$

2.2 One-pot, three-component condensation of aldehydes, terminal alkynes, and amines to synthesize propargylamines

To a flame-dried schlenk pressure tube was added activated catalyst ( $5.0 \mathrm{~mol} \%$, based on the $\mathrm{Cu}^{\mathrm{II}}$ ), terminal alkynes $(0.1 \mathrm{mmol})$, aldehyde $(0.11 \mathrm{mmol})$, morpholine $(0.11 \mathrm{mmol})$ and 1,4 -dioxane $(1 \mathrm{~mL})$. The mixture was stirred at $90{ }^{\circ} \mathrm{C}$ under nitrogen for $12 \mathrm{~h}$. After that, the mixture was centrifuged at $9000 \mathrm{rpm}$ for $5 \mathrm{~min}$, the supernatant was concentrated under vacuum and then purified by column chromatography on silica gel (EtOAc/ petroleum ether, 1/10, v/v) to afford the desired products.

The concentrate was analyzed by ${ }^{1} \mathrm{H}$ NMR to give the conversion.

2.3 The recycle experiments (using the synthesis of 2,3-dihydroquinazolinone as an example)

To a suspension of LMOF 1 (3 mg, $5.0 \mathrm{~mol} \%)$ in $\mathrm{MeOH}(1.0 \mathrm{~mL})$, 2-aminobenzamide (13.6 mg, 
$0.10 \mathrm{mmol})$ and benzaldehyde $(10 \mu \mathrm{L}, 10.6 \mathrm{mg}, 0.10 \mathrm{mmol})$ were added. The reaction was carried out at $70{ }^{\circ} \mathrm{C}$ until the disappearance of the amine. After that, the mixture was centrifuged at $9000 \mathrm{rpm}$ for $5 \mathrm{~min}$, the precipitate was washed with $\mathrm{CH}_{2} \mathrm{Cl}_{2}$ for 3 times and sonicated for $10 \mathrm{~min}$, dried under pressure. Then used for the next run, the supernatant was concentrated under vacuum.

The concentrate was analyzed by ${ }^{1} \mathrm{H}$ NMR to give the conversion.

2.4 Catalyst leaching measurement by ICP-MS (using the synthesis of 2,3-dihydroquinazolinone as an example)

To a suspension of LMOF 1 (3 mg, $5.0 \mathrm{~mol} \%)$ in MeOH (1.0 mL), 2-aminobenzamide (13.6 mg, $0.10 \mathrm{mmol})$ and benzaldehyde $(10 \mu \mathrm{L}, 10.6 \mathrm{mg}, 0.10 \mathrm{mmol})$ were added. The reaction was carried out at $70{ }^{\circ} \mathrm{C}$ until the disappearance of the amine. After that, the mixture was centrifuged at $9000 \mathrm{rpm}$ for $5 \mathrm{~min}$, and the supernatant was concentrated under vacuum then used for ICP-MS analysis.

\section{Representative procedure for dye uptake measurements}

Prior to dye uptake measurement, the LMOF 1 were exchanged with chloroform for two days and then activated under vacuum at room temperature for $4 \mathrm{~h}$ to remove the guest solvents. The activated LMOF $1(50.8 \mathrm{mg}, 87 \mu \mathrm{mol})$ was soaked in a saturated solution of methyl orange in methanol at $50{ }^{\circ} \mathrm{C}$ for 5 days. The resulted samples were filtered and washed with $\mathrm{MeOH}$ several times to remove the dye adsorbed on the surface of crystals. The washed samples were then extracted by Soxhlet extractor in methanol thoroughly until the washings became colorless. The clear orange solution was diluted to $100 \mathrm{~mL}$. Absorption experiments were performed on Lambda 20 UV/Vis Spectrometer.

Creation of a standard curve: (1) The methyl orange $(32.7 \mathrm{mg}$ ) was added to a flask and diluted with methanol to $1000 \mathrm{~mL}$. The solution of methyl orange is stock solution, and 2.5, 5, 10 and $25 \mathrm{~mL}$ stock solution were diluted to $50 \mathrm{~mL}$, respectively. (2) The absorbance of different concentrations of methyl orange was determined by UV/Vis Spectrometer. Data for known concentrations of methyl orange were used to make the standard curve, plotting concentration on the $\mathrm{X}$ axis, and the assay measurement of absorbance on the $\mathrm{Y}$ axis. According to the Beer-Lambert law, the standard curve can be calculated by linear fitting of the data.

$$
A=\log _{10} \frac{I_{0}}{I_{t}}=\log _{10} \frac{1}{T}=K \cdot l \cdot c
$$


4. Table S1. Crystal data and structure refinement for LMOF 1.

\begin{tabular}{|c|c|}
\hline Identification code & LMOF 1 \\
\hline Empirical formula & $\mathrm{C}_{26} \mathrm{H}_{28} \mathrm{CuN}_{6} \mathrm{O}_{6}$ \\
\hline Formula weight & 584.08 \\
\hline Temperature (K) & $150 \mathrm{~K}$ \\
\hline Wavelength $(\AA)$ & 1.54184 \\
\hline Crystal system & Monoclinic \\
\hline Space group & $P 2_{1} / n$ \\
\hline Unit cell dimensions & $\begin{array}{l}\mathrm{a}=10.8380(2) \AA \\
\mathrm{b}=15.4324(3) \AA \\
\mathrm{c}=15.9395(3) \AA \\
\alpha=\gamma=90 ; \beta=95.961(2)\end{array}$ \\
\hline Volume $\left(\AA^{3}\right), \mathrm{Z}$ & $2651.57(9), 4$ \\
\hline Density (calculated) $\left(\mathrm{mg} / \mathrm{m}^{3}\right)$ & 1.463 \\
\hline Absorption coefficient $\left(\mathrm{mm}^{-1}\right)$ & 1.612 \\
\hline $\mathrm{F}(000)$ & 1212.0 \\
\hline$\theta$ range for data collection $\left({ }^{\circ}\right)$ & 7.996 to 144.892 \\
\hline Limiting indices & $\begin{array}{l}-11 \leq \mathrm{h} \leq 13 \\
-13 \leq \mathrm{k} \leq 19 \\
-17 \leq 1 \leq 19\end{array}$ \\
\hline Reflections collected & 12744 \\
\hline Independent reflections & $5128\left[\mathrm{R}_{\text {int }}=0.0253, \mathrm{R}_{\text {sigma }}=0.0289\right]$ \\
\hline Completeness to theta & $97 \%$ \\
\hline Data / restraints / parameters & $5128 / 41 / 370$ \\
\hline Goodness-of-fit on $\mathrm{F}^{\wedge} 2$ & 1.049 \\
\hline Final $R$ indices [I $>2 \operatorname{sigma}(\mathrm{I})]$ & $\mathrm{R}_{1}=0.0667, \mathrm{wR}_{2}=0.1987$ \\
\hline $\mathrm{R}$ indices (all data) & $\mathrm{R}_{1}=0.0840, \mathrm{wR}_{2}=0.2214$ \\
\hline Absolute structure parameter & $0.05(5)$ \\
\hline Largest diff. peak and hole $\left(\mathrm{e} . \AA^{-3}\right)$ & $1.88 /-1.13$ \\
\hline
\end{tabular}

5. Table S2. Selected bond lengths [Ǻ] and angles $\left[{ }^{\circ}\right]$ for LMOF 1

\begin{tabular}{llll}
\hline $\mathrm{Cu}(1)-\mathrm{O}(1) \# 1$ & $2.004(2)$ & $\mathrm{Cu}(2)-\mathrm{O}(2) \# 4$ & $1.983(2)$ \\
$\mathrm{Cu}(1)-\mathrm{O}(1)$ & $2.004(2)$ & $\mathrm{Cu}(2)-\mathrm{O}(2)$ & $1.983(2)$ \\
$\mathrm{Cu}(1)-\mathrm{N}(1) \# 2$ & $1.999(3)$ & $\mathrm{Cu}(2)-\mathrm{N}(3) \# 5$ & $1.979(3)$ \\
$\mathrm{Cu}(1)-\mathrm{N}(1) \# 3$ & $1.999(3)$ & $\mathrm{Cu}(2)-\mathrm{N}(3) \# 2$ & $1.979(3)$ \\
$\mathrm{O}(1) \# 1-\mathrm{Cu}(1)-\mathrm{O}(1)$ & $180.0(13)$ & $\mathrm{O}(2) \# 4-\mathrm{Cu}(2)-\mathrm{O}(2)$ & 180.0 \\
$\mathrm{~N}(1) \# 2-\mathrm{Cu}(1)-\mathrm{O}(1) \# 1$ & $89.33(11)$ & $\mathrm{N}(3) \# 3-\mathrm{Cu}(2)-\mathrm{O}(2) \# 4$ & $89.88(11)$ \\
$\mathrm{N}(1) \# 3-\mathrm{Cu}(1)-\mathrm{O}(1) \# 1$ & $90.67(11)$ & $\mathrm{N}(3) \# 3-\mathrm{Cu}(2)-\mathrm{O}(2)$ & $90.11(11)$ \\
$\mathrm{N}(1) \# 2-\mathrm{Cu}(1)-\mathrm{O}(1)$ & $90.67(11)$ & $\mathrm{N}(3) \# 5-\mathrm{Cu}(2)-\mathrm{O}(2) \# 4$ & $90.12(11)$ \\
$\mathrm{N}(1) \# 3-\mathrm{Cu}(1)-\mathrm{O}(1)$ & $89.33(11)$ & $\mathrm{N}(3) \# 5-\mathrm{Cu}(2)-\mathrm{O}(2)$ & $89.89(11)$ \\
$\mathrm{N}(1) \# 2-\mathrm{Cu}(1)-\mathrm{N}(1) \# 3$ & 180.0 & $\mathrm{~N}(3) \# 3-\mathrm{Cu}(2)-\mathrm{N}(3) \# 5$ & 180.0 \\
\hline
\end{tabular}

Symmetry transformations used to generate equivalent atoms:

$\begin{array}{ll}\text { \#1 1-x,2-y,1-z } & \# 2-1 / 2+x, 3 / 2-y,-1 / 2+z\end{array}$ \#3 3/2-x,1/2+y,3/2-z 
6. Table S3. Hydrogen Bond Lengths $(\AA)$ and Bond Angles $\left({ }^{\circ}\right)$

\begin{tabular}{lllll}
\hline $\mathrm{D}-\mathrm{H} \cdots \mathrm{A}$ & $\mathrm{d}(\mathrm{D}-\mathrm{H})$ & $\mathrm{d}(\mathrm{H} \cdots \mathrm{A})$ & $\mathrm{d}(\mathrm{D} \cdots \mathrm{A})$ & $\angle \mathrm{DHA}$ \\
\hline $\mathrm{C}(4)-\mathrm{H}(4) \cdots \mathrm{O}(5)$ & 0.93 & 2.58 & $3.265(5)$ & 131 \\
$\mathrm{C}(4)-\mathrm{H}(4) \cdots \mathrm{O}(2)$ & 0.93 & 2.47 & $2.914(4)$ & 109 \\
$\mathrm{C}(6)-\mathrm{H}(6) \cdots \mathrm{O}(1)$ & 0.93 & 2.51 & $2.931(4)$ & 108 \\
$\mathrm{C}(13)-\mathrm{H}(13) \cdots \mathrm{O}(6)$ & 0.93 & 2.38 & $3.064(6)$ & 131 \\
$\mathrm{C}(16)-\mathrm{H}(16) \cdots \mathrm{O}(3)$ & 0.93 & 2.52 & $3.444(4)$ & 176 \\
$\mathrm{C}(18)-\mathrm{H}(18) \cdots \mathrm{O}(5)$ & 0.93 & 2.51 & $3.387(5)$ & 158 \\
$\mathrm{C}(19)-\mathrm{H}(19) \cdots \mathrm{O}(2)$ & 0.93 & 2.60 & $2.979(5)$ & 105 \\
$\mathrm{C}(20)-\mathrm{H}(20) \cdots \mathrm{O}(4)$ & 0.93 & 2.27 & $3.126(6)$ & 153 \\
$\mathrm{C}(22)-\mathrm{H}(22 \mathrm{C}) \cdots \mathrm{O}(5)$ & 0.96 & 2.38 & $2.791(8)$ & 105 \\
$\mathrm{C}(23)-\mathrm{H}(23) \cdots \mathrm{O}(3)$ & 0.93 & 2.53 & $3.383(10)$ & 153 \\
$\mathrm{C}(26)-\mathrm{H}(26 \mathrm{~A}) \cdots \mathrm{O}(3)$ & 0.96 & 2.60 & $3.427(11)$ & 145 \\
$\mathrm{C}(26)-\mathrm{H}(26 \mathrm{~B}) \cdots \mathrm{O}(6)$ & 0.96 & 2.43 & $3.363(12)$ & 163 \\
\hline
\end{tabular}

7. Figure S1 S4. Additional X-ray crystallographic structures

7.1 Figure $S 1$. The asymmetric unit

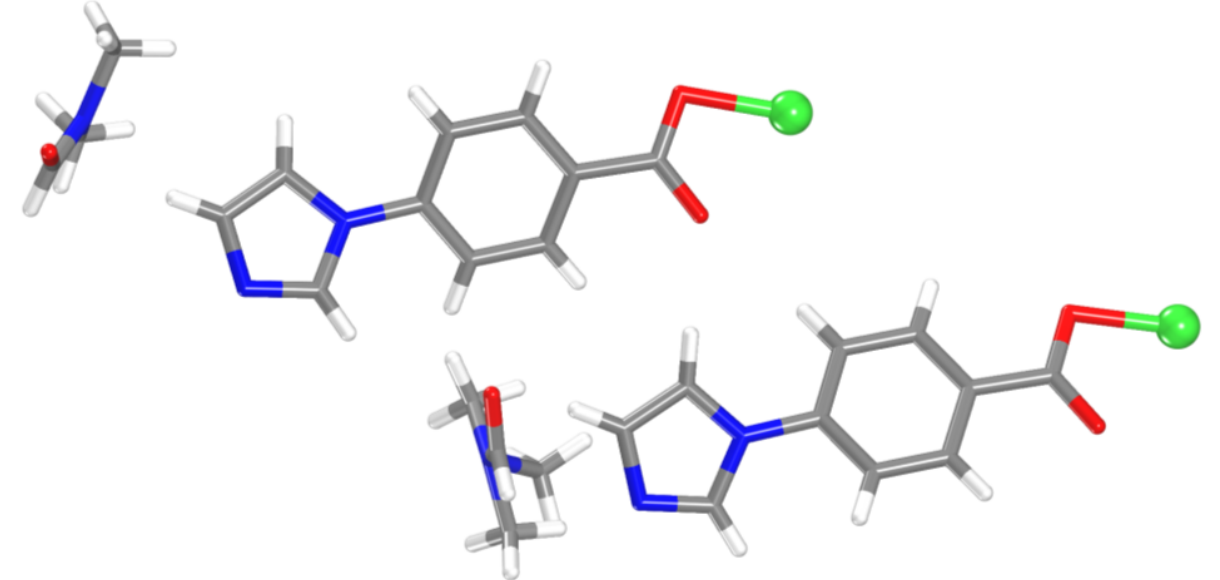

7.2 Figure S2. The structure of 4-connected square-planar geometry of $\mathrm{Cu}^{\mathrm{II}}$

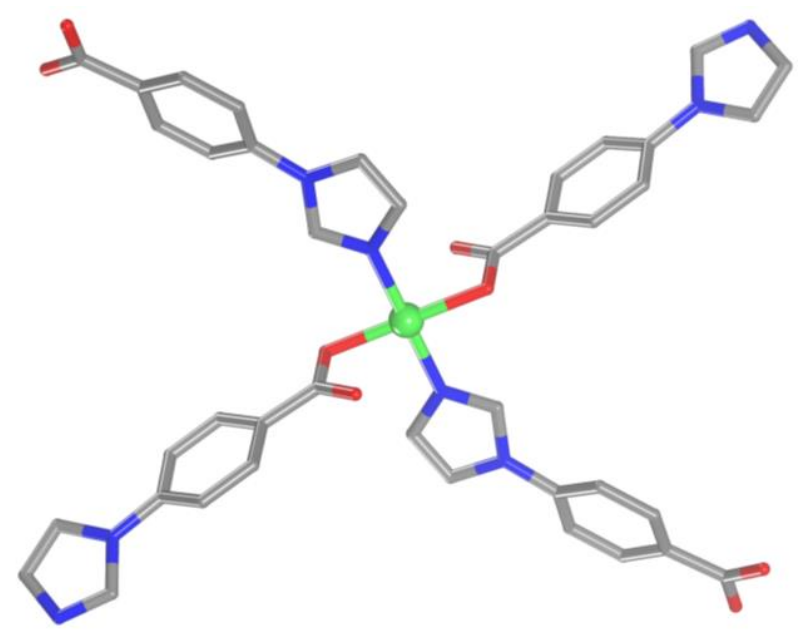


7.3 Figure S3. The single rhombic layer

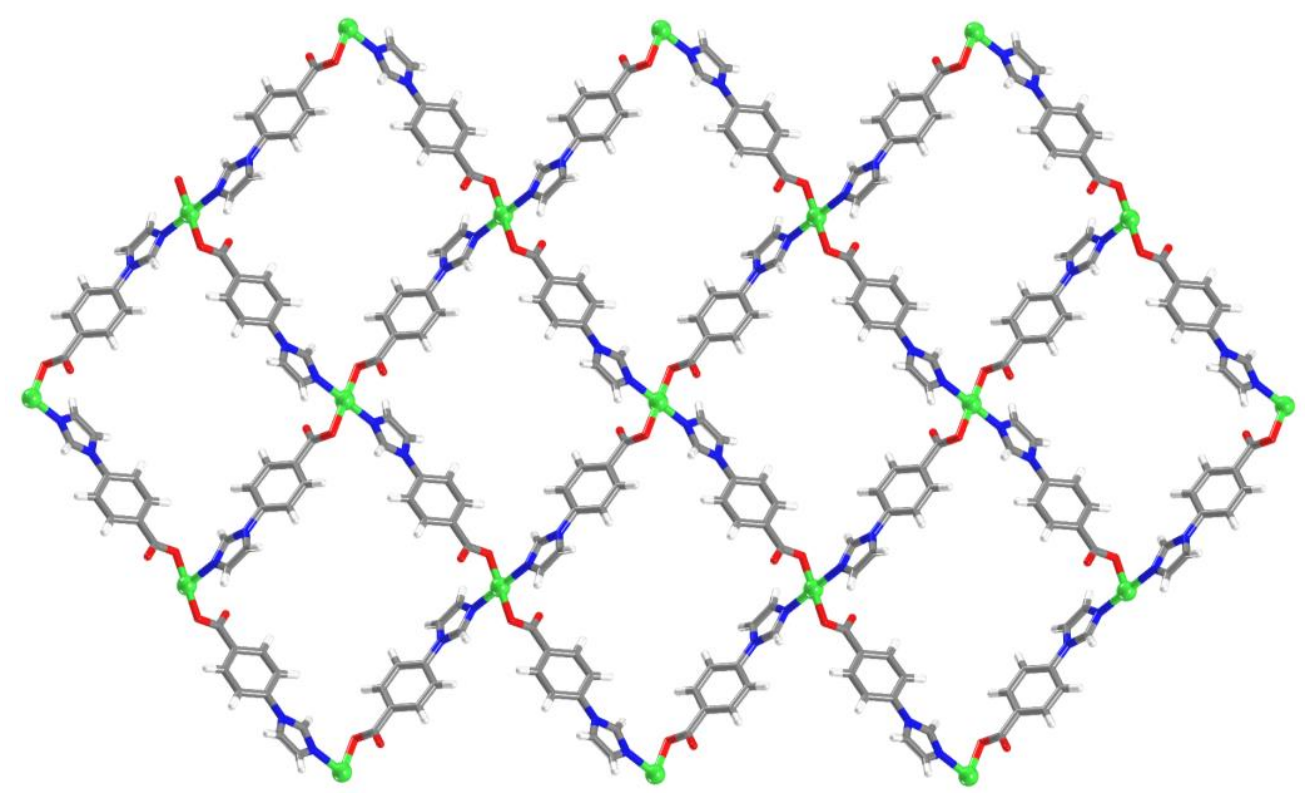

7.4 Figure S4. The ABC staggered stacking model of the single rhombic layers

a)

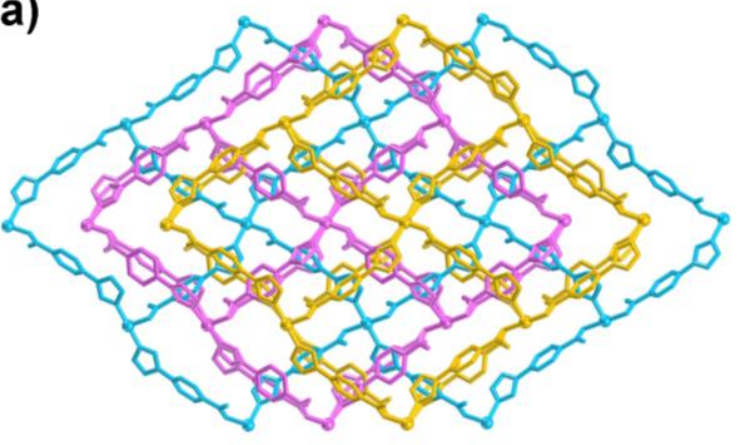

III

b)

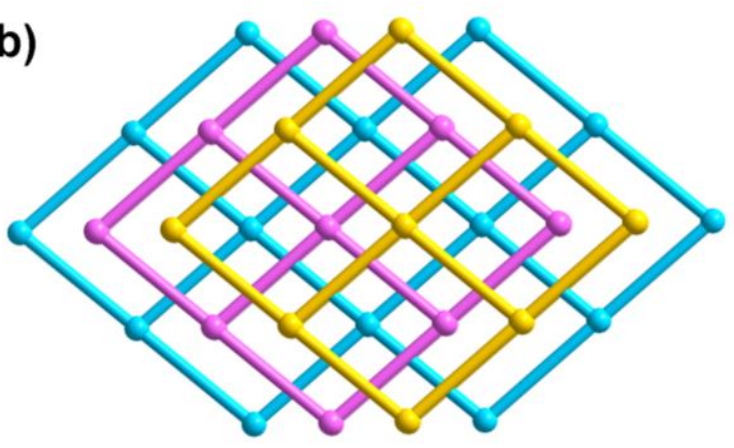

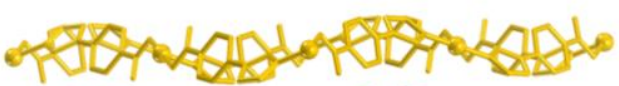
S

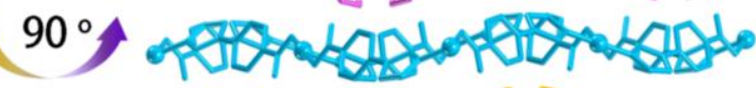
prop ra

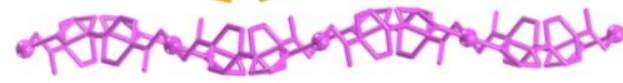

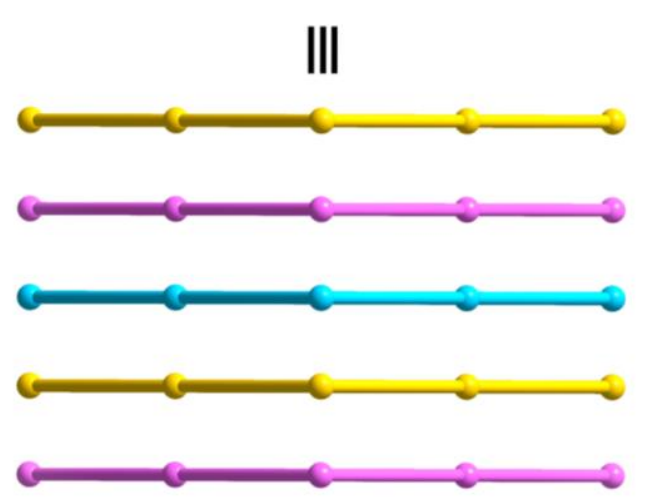


8. Figure S5. TGA curve

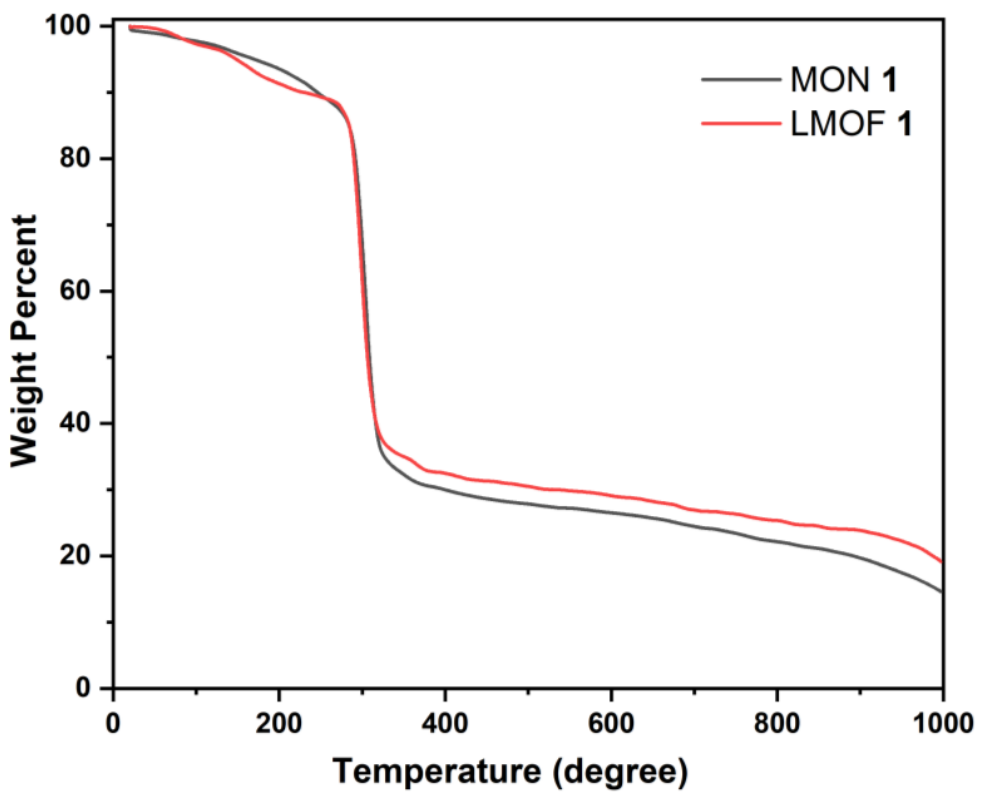

9. Figure S6 S9. SEM and Microscope images

9.1 Figure S6. SEM image of LMOF 1 and MON 1

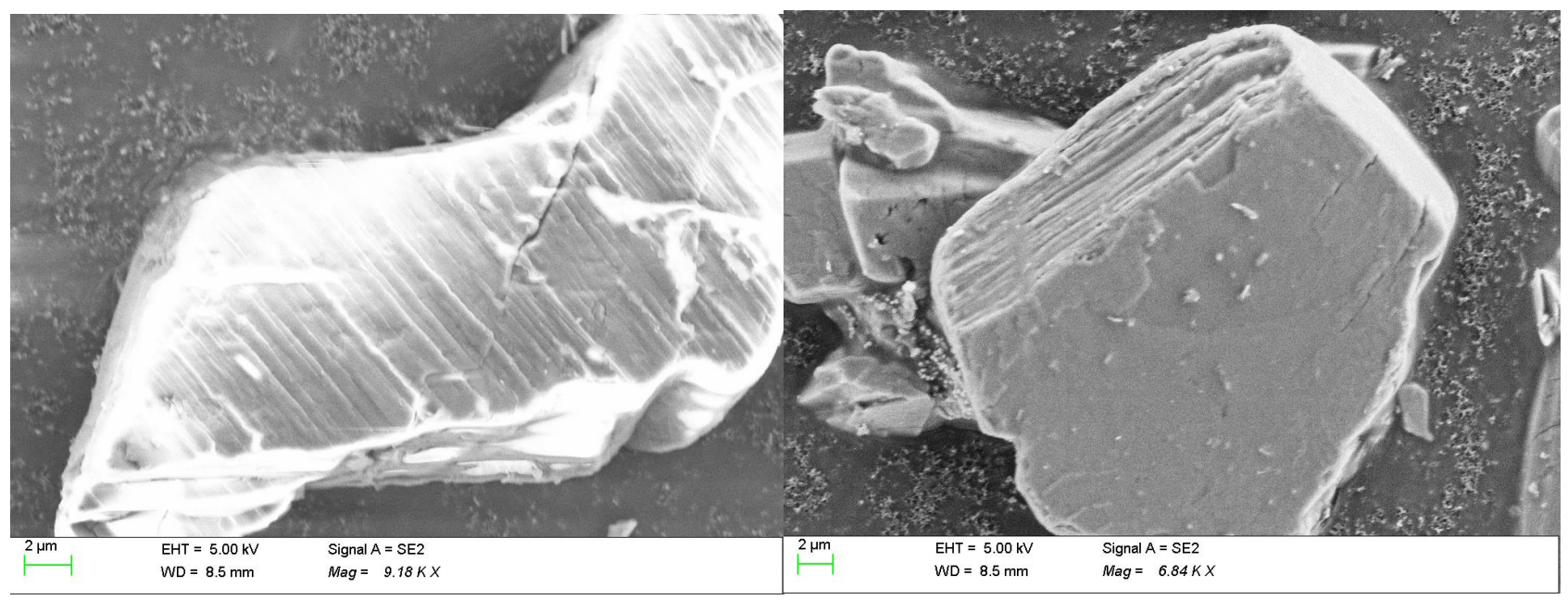

\subsection{Figure S7 Microscope images of LMOF 1}

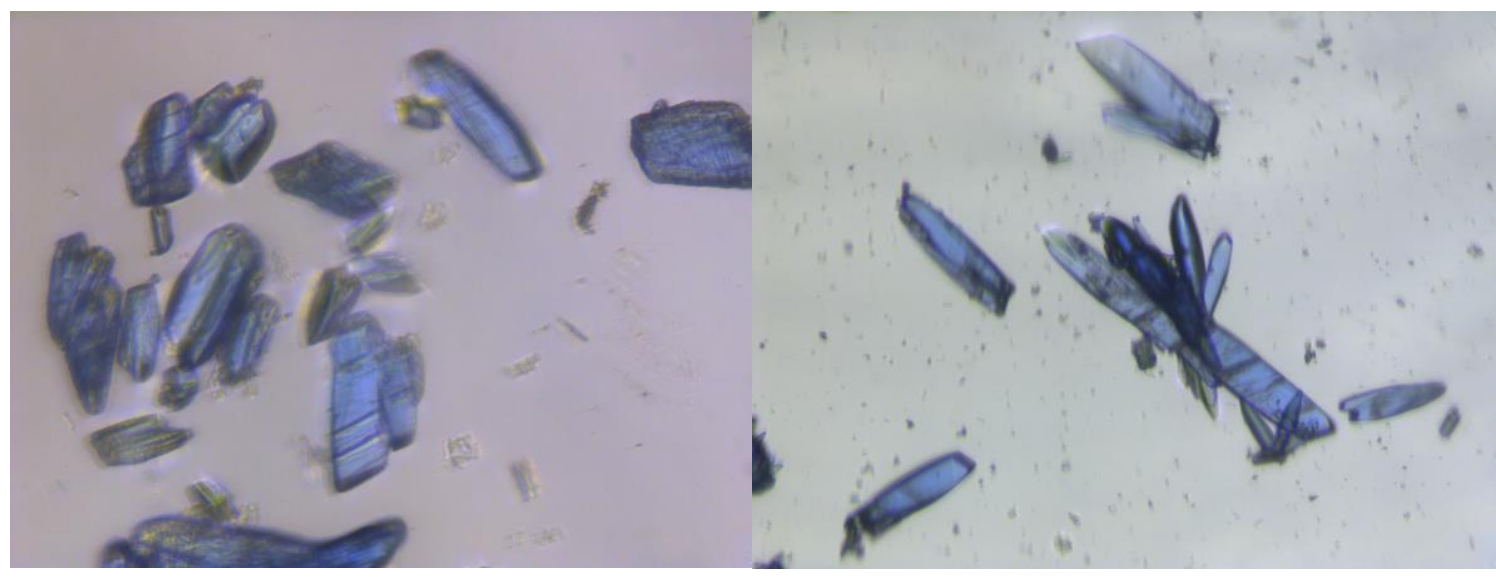


9.3 Figure S8. SEM image of LMOF 1 after ultrasound treatment

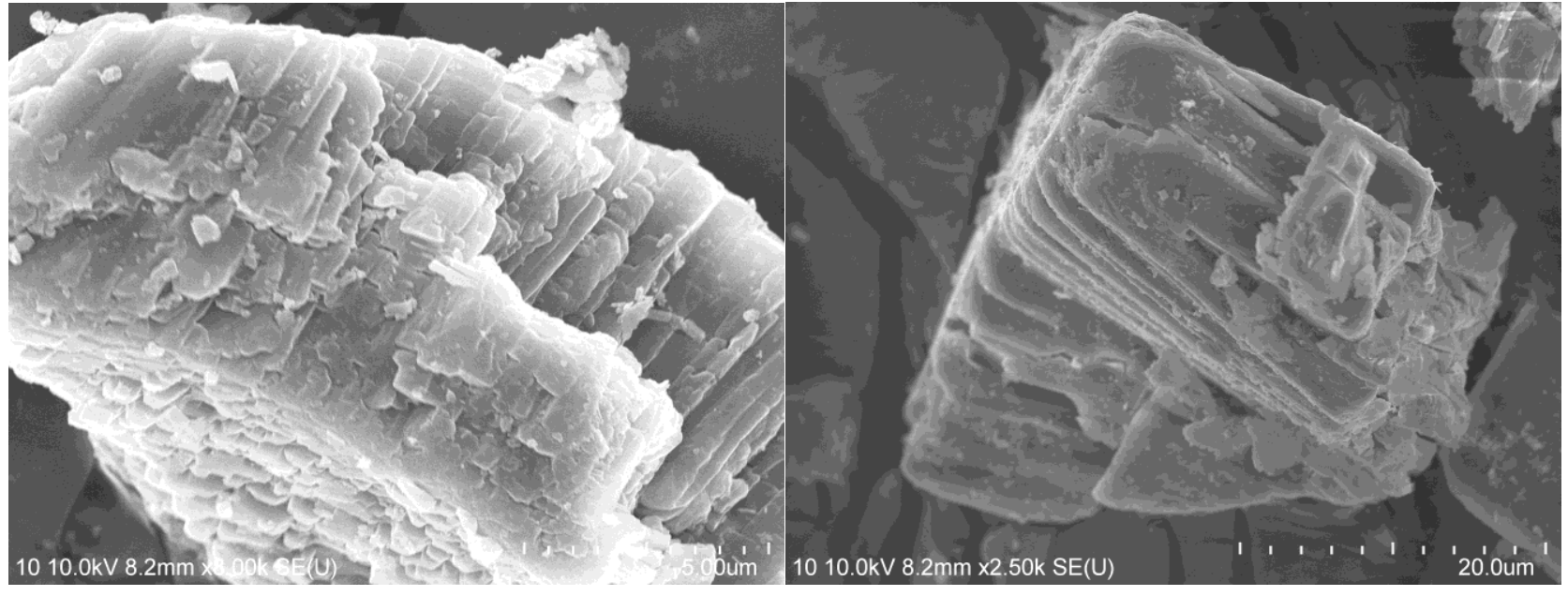

\subsection{Figure S9. SEM image of MON 1}

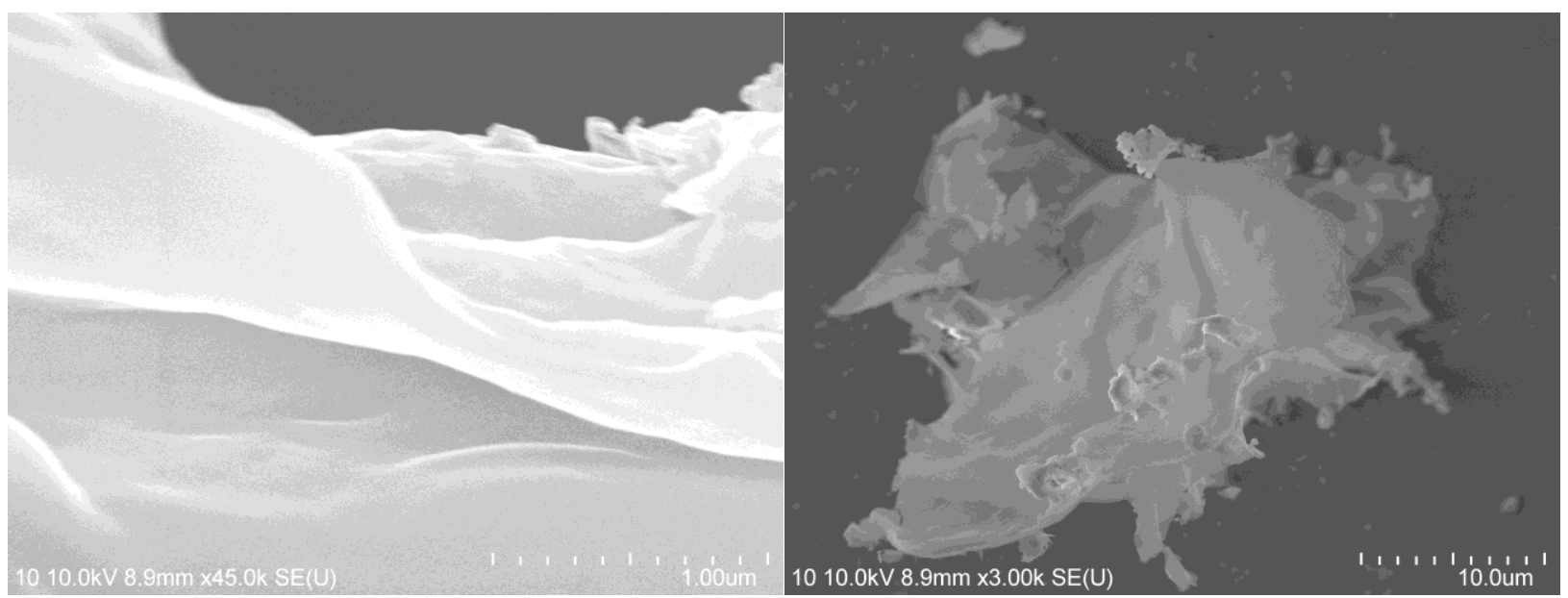


10. Figure S10. TEM image of MON 1

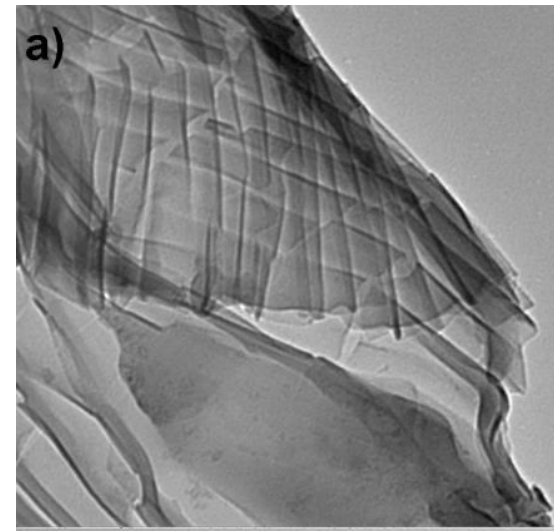

b)

c)

\section{$100 \mathrm{~nm}$}

$※$ Figure $\mathrm{S} 10 \mathrm{c}$ is the TEM images of the MON 1 after 5 catalytic runs 


\section{Figure S11. AFM image of MON 1}
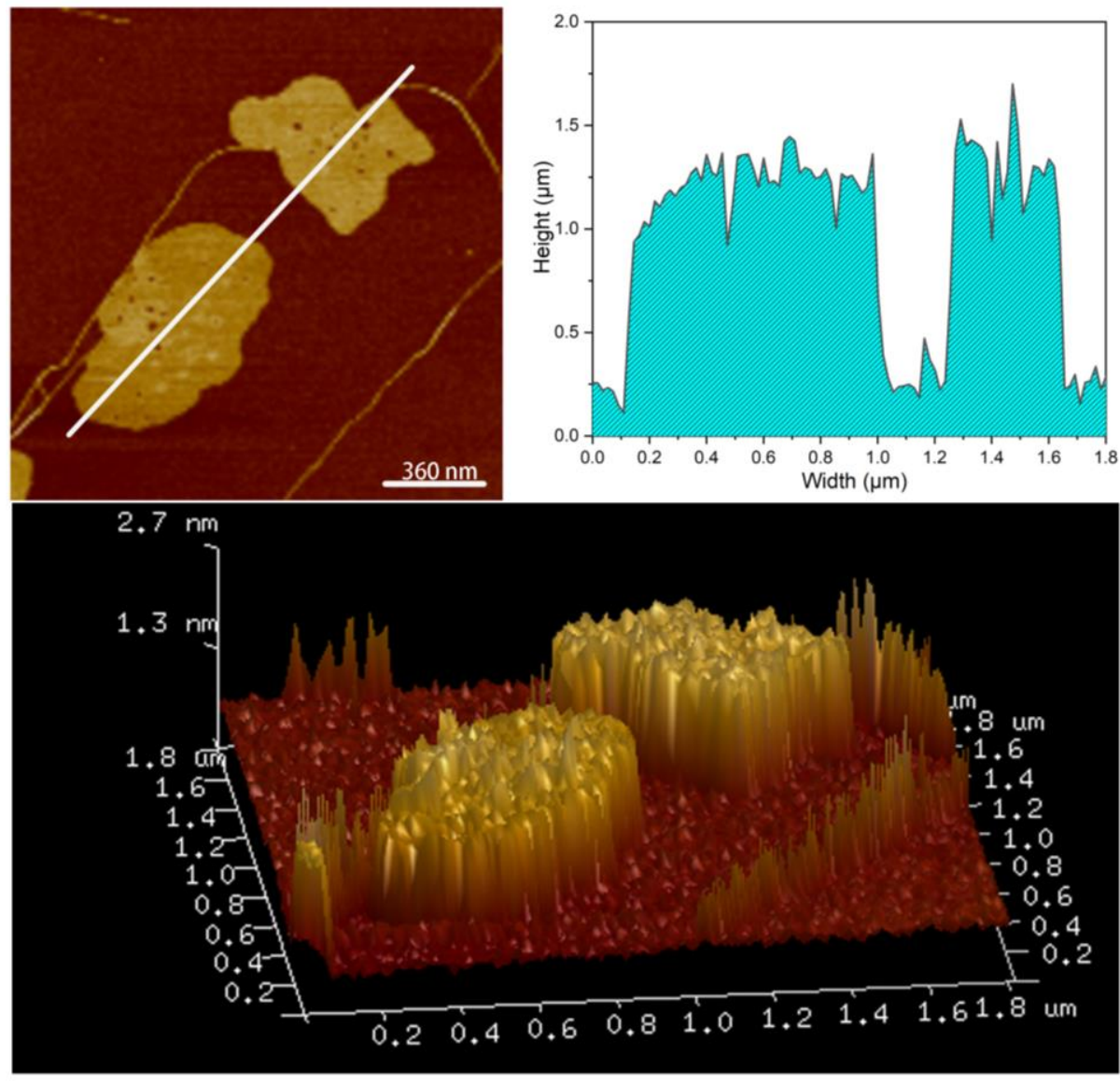

\section{Figure S12. XPS spectra}
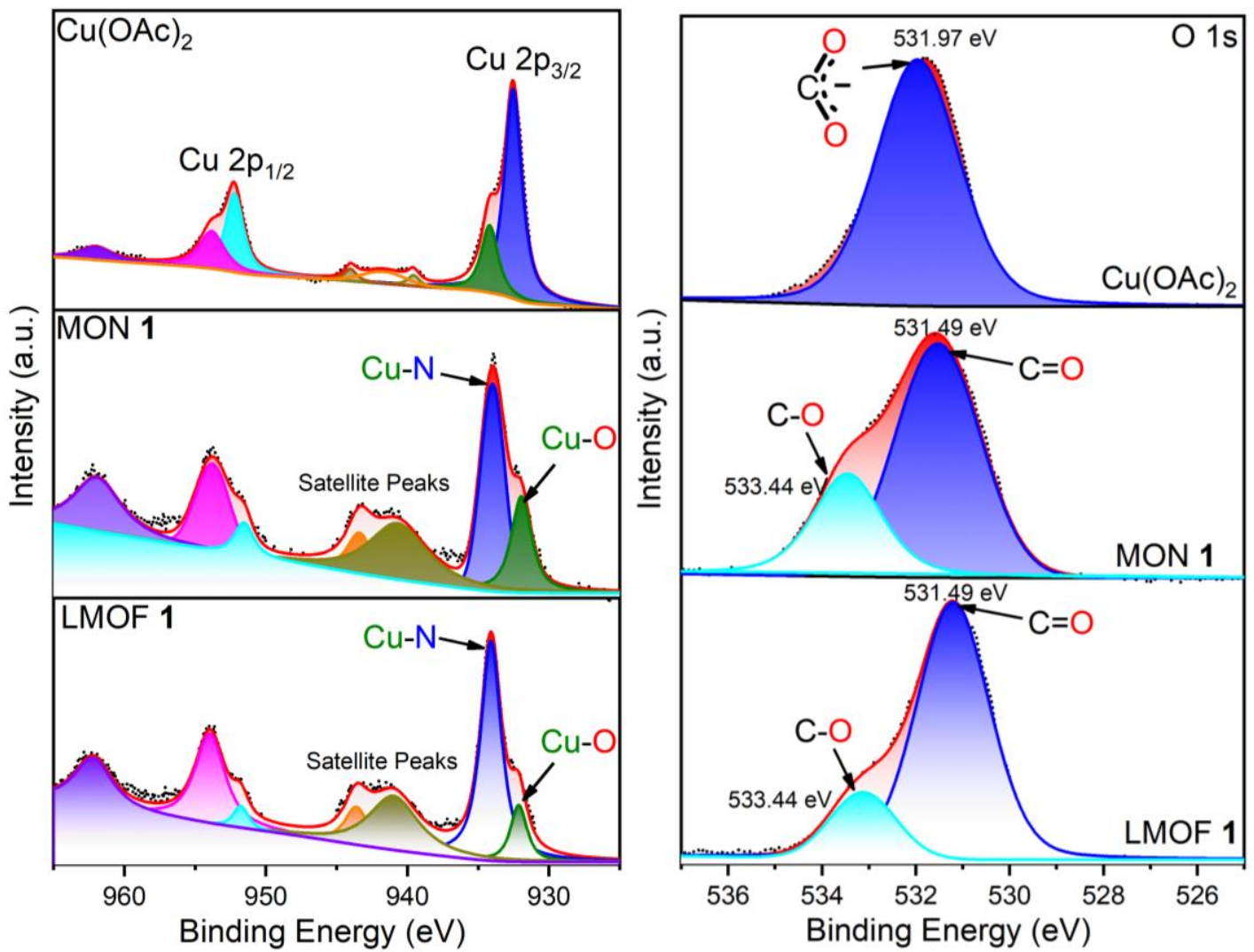


\section{Figure S13. Dye adsorption}

a)
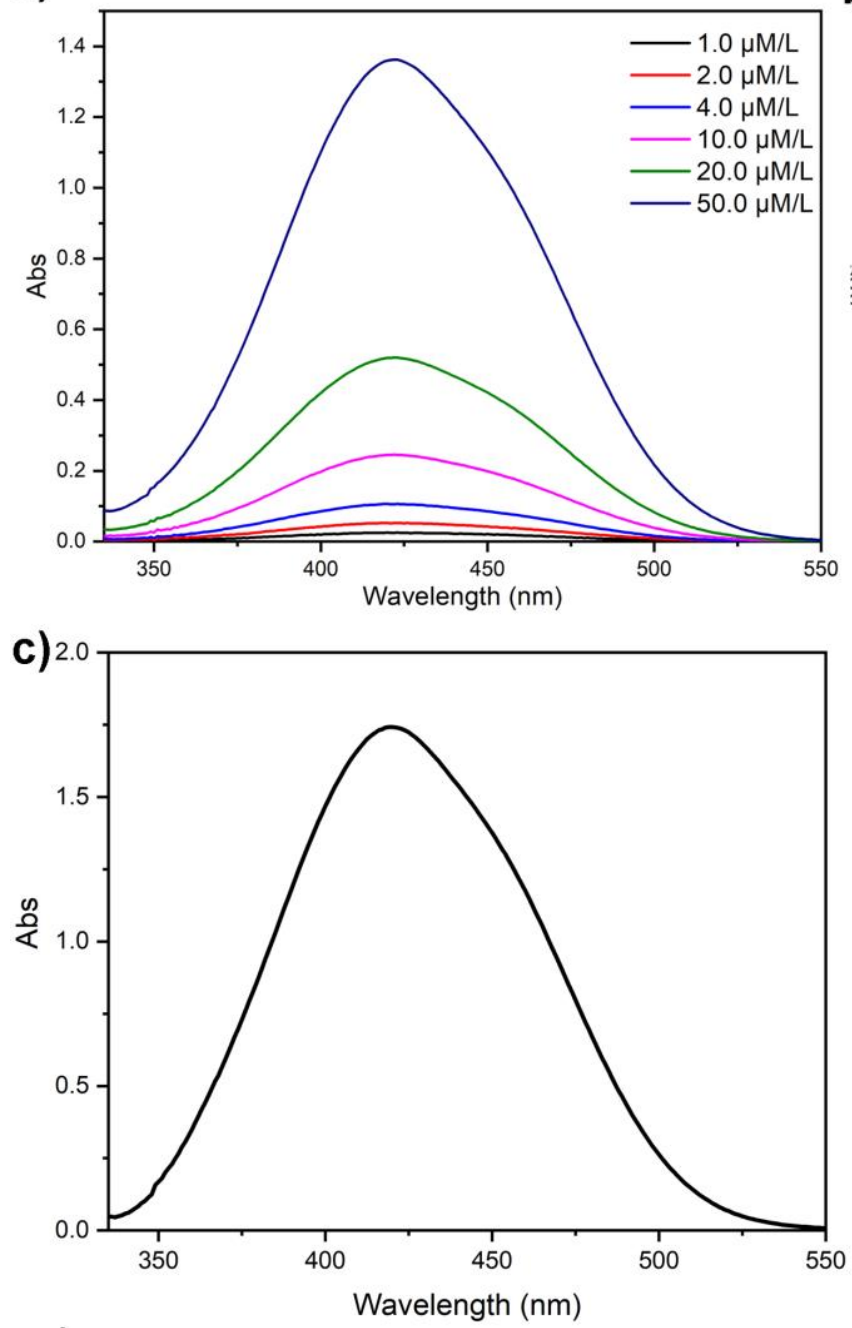

b)

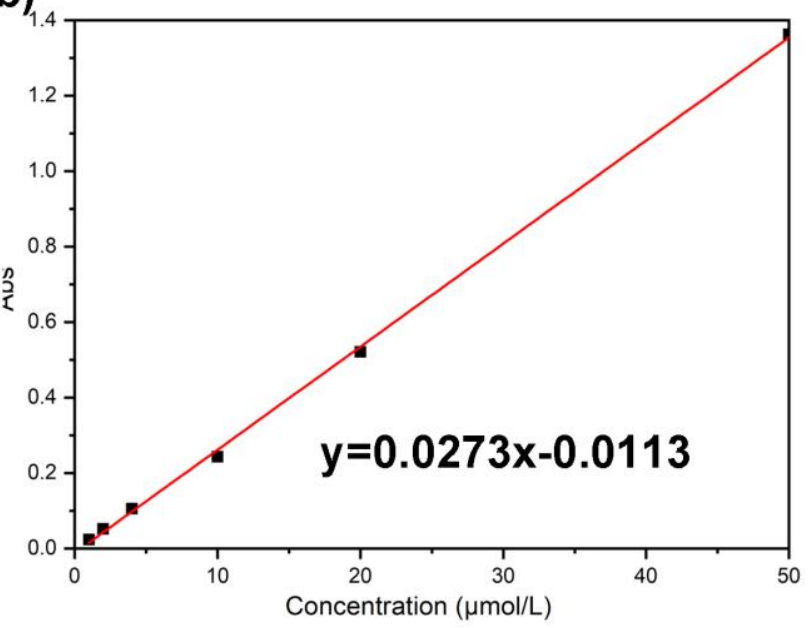

d)

\begin{tabular}{|l|c|}
\hline & $\begin{array}{c}\text { The number of dye uptake } \\
\text { (per formula) }\end{array}$ \\
\hline Methyl Orange & 0.59 \\
\hline
\end{tabular}

f)

e)
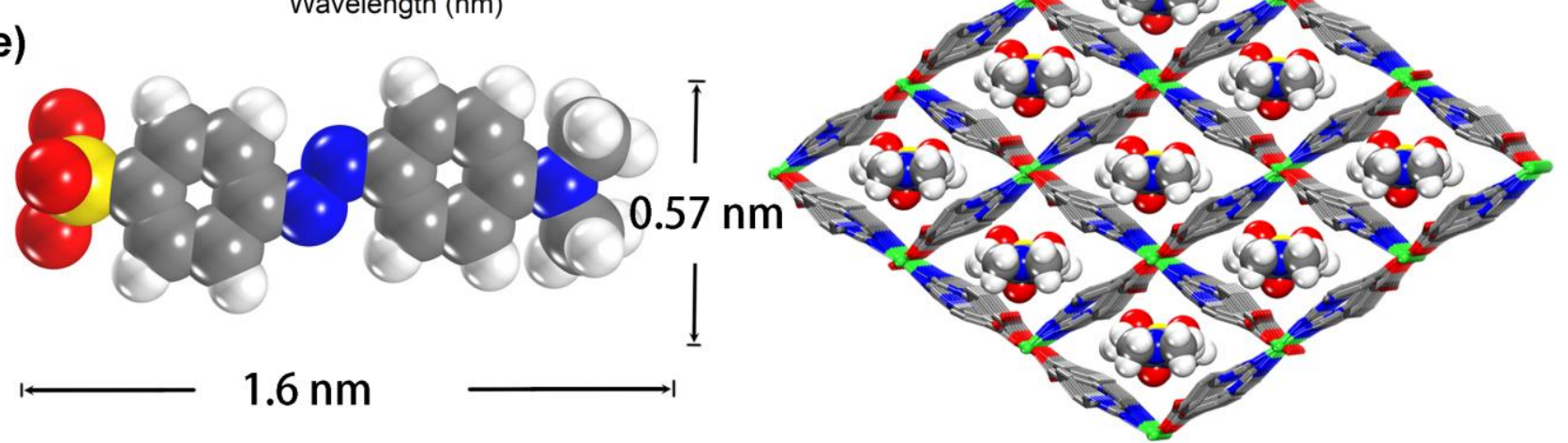

a) UV-Vis absorption of methyl orange with different concentrations, and b) the standard curve. c) The UV-Vis absorption of methyl orange adsorbed in LMOF 1 which was extracted by Soxhlet extractor in methanol. The concentration of the dye molecules was determined by comparing standard curve. $d$ ) The number of the dye uptakes. The number of methyl orange uptakes was calculated by dividing the amount of dye per unit cell by the void space inside the unit cell. e) The size of methyl orange calculated by Materials Studio (2019), and f) the simulation of the uptake of methyl orange in LMOF 1. 
14. Figure S14. The plausible mechanism for the formation of 2,3-dihydroquinazolinone

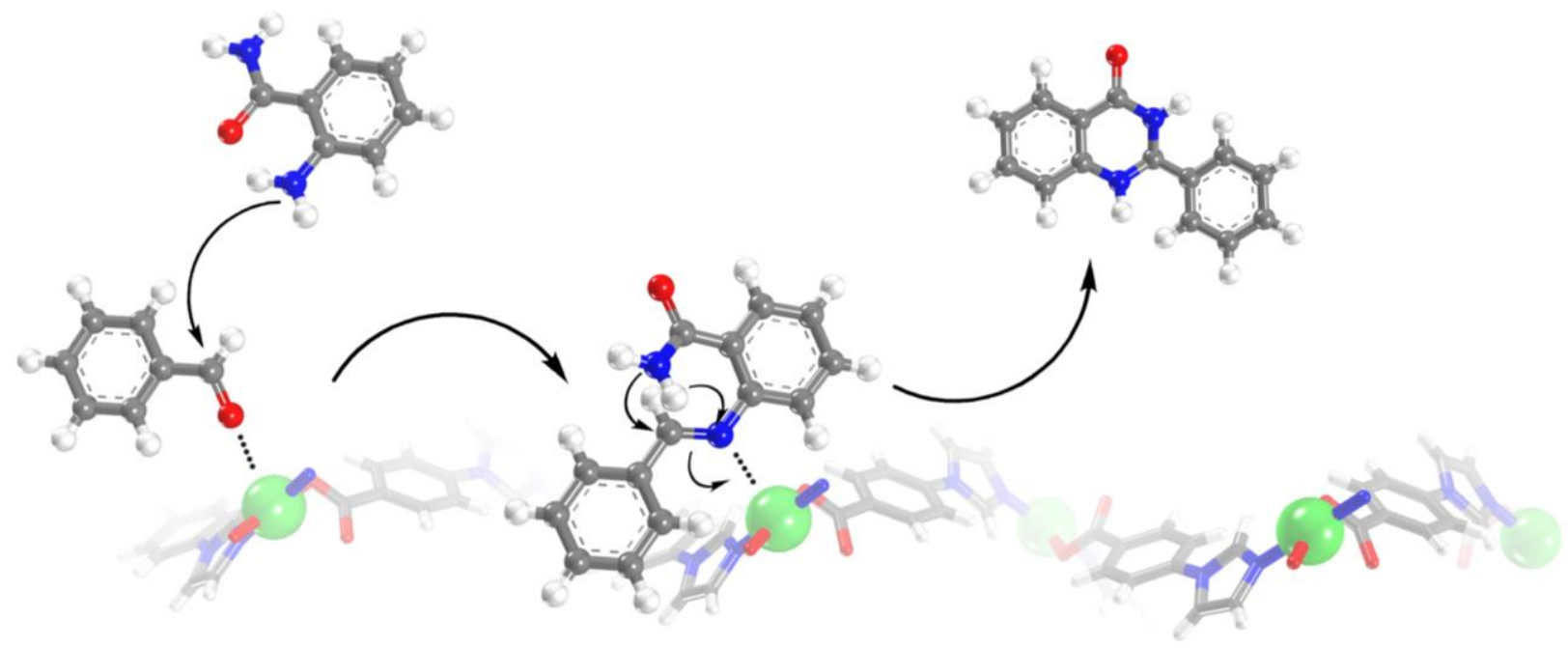

15. Figure S15. The size of the sterically aromatic aldehydes and the corresponding 2,3dihydroquinazolinone, propargylamines
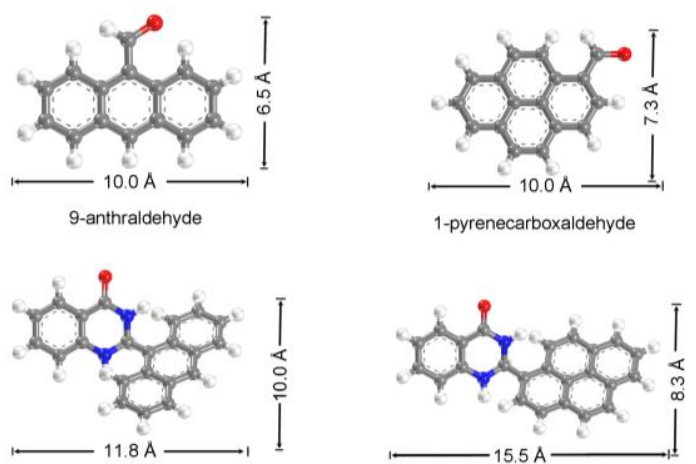
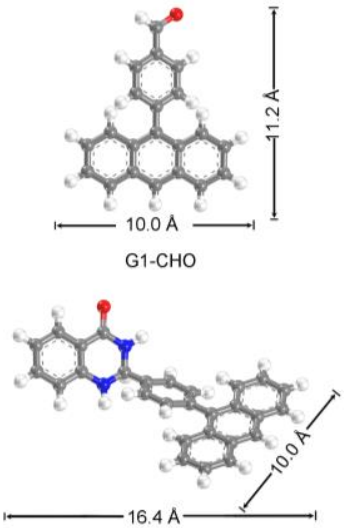
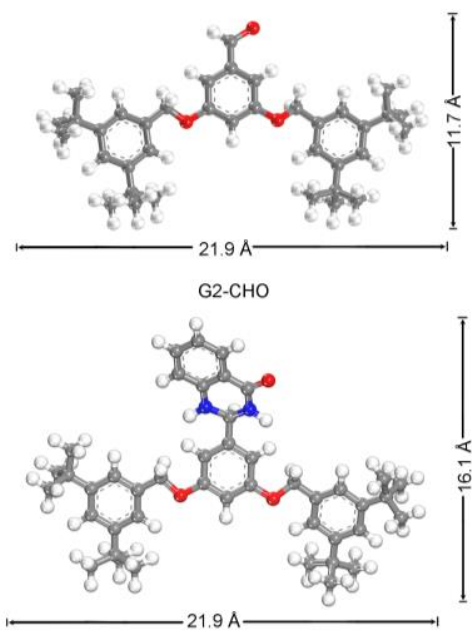

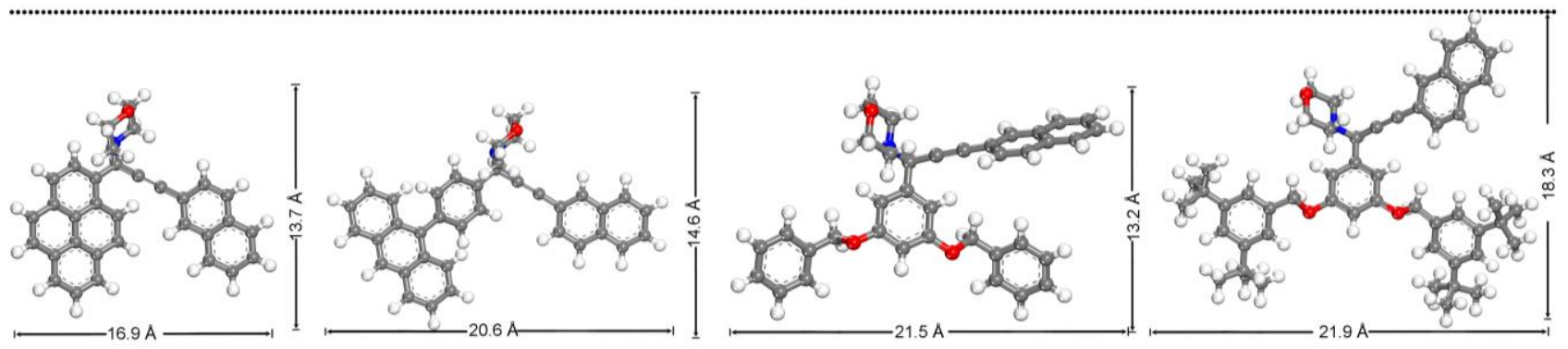

The size of these sterically aromatic aldehydes, 2,3-dihydroquinazolinone and propargylamines were calculated by Materials Studio (2019), and were fully optimized using MS Forcite molecular dynamics module (universal force fields, Ewald summations) method. 
16. Additional catalytic results

16.1 Table S4. Optimization of the reaction conditions for acetalization reaction<smiles>NC(=O)c1ccccc1N</smiles><smiles>O=Cc1ccccc1</smiles><smiles></smiles><smiles>O=C1NC(c2ccccc2)Nc2ccccc21</smiles>

\begin{tabular}{llllll}
\hline Entry & solvent & Loading $(\mathrm{mol} \%)$ & $\mathrm{T}\left({ }^{\circ} \mathrm{C}\right)$ & additive & Conversion $(\%)$ \\
\hline 1 & $\mathrm{DCM}$ & 10 & 60 & $\mathrm{MgSO}_{4}$ & 48 \\
2 & $\mathrm{DCE}$ & 10 & 60 & $\mathrm{MgSO}_{4}$ & 52 \\
3 & $\mathrm{CHCl}_{3}$ & 10 & 60 & $\mathrm{MgSO}_{4}$ & 39 \\
4 & Toluene & 10 & 60 & $\mathrm{MgSO}_{4}$ & $\mathrm{ND}$ \\
5 & $\mathrm{DME}$ & 10 & 60 & $\mathrm{MgSO}_{4}$ & $<10$ \\
6 & $1,4-$ dioxane & 10 & 60 & $\mathrm{MgSO}_{4}$ & $<10$ \\
7 & $\mathrm{EA}$ & 10 & 60 & $\mathrm{MgSO}_{4}$ & $\mathrm{ND}$ \\
8 & $\mathrm{THF}$ & 10 & 60 & $\mathrm{MgSO}_{4}$ & 56 \\
9 & $\mathrm{MeCN}$ & 10 & 60 & $\mathrm{MgSO}_{4}$ & 67 \\
10 & $\mathrm{MTBE}$ & 10 & 60 & $\mathrm{MgSO}_{4}$ & $<10$ \\
11 & $\mathrm{Hexane}$ & 10 & 60 & $\mathrm{MgSO}_{4}$ & $\mathrm{ND}$ \\
12 & $\mathrm{MeOH}$ & 10 & 60 & $\mathrm{MgSO}_{4}$ & 89 \\
13 & $\mathrm{MeOH}$ & 5 & 60 & $\mathrm{MgSO}_{4}$ & 87 \\
14 & $\mathrm{MeOH}$ & 2 & 60 & $\mathrm{MgSO}_{4}$ & 75 \\
15 & $\mathrm{MeOH}$ & 1 & 60 & $\mathrm{MgSO}_{4}$ & 64 \\
16 & $\mathrm{MeOH}$ & 5 & $\mathrm{rt}$ & $\mathrm{MgSO}_{4}$ & $\mathrm{ND}$ \\
17 & $\mathrm{MeOH}$ & 5 & 40 & $\mathrm{MgSO}_{4}$ & 59 \\
18 & $\mathrm{MeOH}$ & 5 & 60 & $4 \AA \mathrm{MS}$ & 73 \\
19 & $\mathrm{MeOH}$ & 5 & 70 & $\mathrm{MgSO}_{4}$ & 98 \\
20 & $\mathrm{MeOH}$ & 5 & 90 & $\mathrm{MgSO}_{4}$ & 92 \\
\hline
\end{tabular}

※The conversion was calculated according to ${ }^{1} \mathrm{H}$ NMR of the reaction solutions. 


\begin{tabular}{|c|c|c|c|c|}
\hline Entry & solvent & Loading (mol\%) & $\mathrm{T}\left({ }^{\circ} \mathrm{C}\right)$ & Conversion $(\%)$ \\
\hline 1 & DCM & 10 & 60 & 37 \\
\hline 2 & DCE & 10 & 60 & 42 \\
\hline 3 & $\mathrm{CHCl}_{3}$ & 10 & 60 & 49 \\
\hline 4 & Toluene & 10 & 60 & 57 \\
\hline 5 & DME & 10 & 60 & 76 \\
\hline 6 & $\mathrm{MeOH}$ & 10 & 60 & ND \\
\hline 7 & EA & 10 & 60 & ND \\
\hline 8 & THF & 10 & 60 & $<10$ \\
\hline 9 & $\mathrm{MeCN}$ & 10 & 60 & 27 \\
\hline 10 & MTBE & 10 & 60 & 69 \\
\hline 11 & Hexane & 10 & 60 & 47 \\
\hline 12 & 1, 4-dioxane & 10 & 60 & 83 \\
\hline 13 & 1, 4-dioxane & 5 & 60 & 82 \\
\hline 14 & 1, 4-dioxane & 2 & 60 & 61 \\
\hline 15 & 1, 4-dioxane & 1 & 60 & 37 \\
\hline 16 & 1, 4-dioxane & 5 & $\mathrm{rt}$ & ND \\
\hline 17 & 1, 4-dioxane & 5 & 40 & 44 \\
\hline 19 & 1, 4-dioxane & 5 & 70 & 85 \\
\hline 20 & 1, 4-dioxane & 5 & 90 & 90 \\
\hline
\end{tabular}

※The conversion was calculated according to ${ }^{1} \mathrm{H}$ NMR of the reaction solutions. 
17. Table S6. Summary of the solvents used for ultrasonic exfoliation of LMOFs into MONs

\begin{tabular}{|c|c|c|c|}
\hline Entry & MOF & solvent & Ref. \\
\hline 1 & {$[\mathrm{Cu}(1-5)(\mathrm{DMF})]_{\mathrm{n}}$} & $\mathrm{DMF}$ or $\mathrm{MeCN}$ & $\begin{array}{l}\text { Inorg. Chem. 2019, 58, 10837- } \\
10845\end{array}$ \\
\hline 2 & $\begin{array}{l}\text { M-MOF-Ru(tpyCOO })_{2} \\
(\mathrm{M}=\mathrm{Mn}, \mathrm{Co}, \mathrm{Ni} \text { or } \mathrm{Zn})\end{array}$ & EtOH & $\begin{array}{llll}\text { Inorg. Chem. } & \text { 2020, } & 59, \\
2379-2386 & & \end{array}$ \\
\hline 3 & $\mathrm{Zn}_{2}(\mathrm{bim})_{4}$ & $\begin{array}{l}\text { ionic liquid and water mixed sol- } \\
\text { vents }\end{array}$ & $\begin{array}{l}\text { ACS } \quad \text { Sustainable Chem. Eng. } \\
\text { 2020, } 8,2167-2175\end{array}$ \\
\hline 4 & $\begin{array}{l}\mathrm{M}(1)(\mathrm{DMF}) \\
(\mathrm{M}=\mathrm{Zn} \text { or } \mathrm{Cu})\end{array}$ & $\begin{array}{l}\text { DMF, } \mathrm{MeCN} \text {, Acetone, EtOH, or } \\
\text { DMF, } \mathrm{H}_{2} \mathrm{O} \text { mixed solvents }\end{array}$ & $\begin{array}{l}\text { Chem. Commun., 2016, 52, } 10474- \\
10477\end{array}$ \\
\hline 5 & ZSB-1 & $n$-hexyl alcohol & Small 2018, 1703873 \\
\hline 6 & NiCo-MOFs & $\begin{array}{l}\text { DMF, EtOH and } \mathrm{H}_{2} \mathrm{O} \text { mixed sol- } \\
\text { vents }\end{array}$ & Nat. Energy. 2016, 1, 16184. \\
\hline 7 & $\begin{array}{l}\mathrm{Cu}(1)(\mathrm{DMF}) \\
\mathrm{Cu}(2)(\mathrm{DMF})\end{array}$ & $\begin{array}{l}\text { DMF, NMP, MeCN, Diethyl } \\
\text { Ether, } \mathrm{H}_{2} \mathrm{O}\end{array}$ & $\begin{array}{l}\text { Chem. Eur. J. 2018, 24, 17986- } \\
17996\end{array}$ \\
\hline 8 & $\mathrm{Zn}_{2}(\mathrm{Bim})_{3}$ & $\begin{array}{l}\text { methanol and } n \text {-propanol mixture } \\
\text { solvents }\end{array}$ & $\begin{array}{l}\text { Angew. Chem. Int. Ed. 2017, 56, } \\
9757 \text {-9761 }\end{array}$ \\
\hline 9 & Eu-MOF & $\mathrm{MeCN}$ & $\begin{array}{l}\text { J. Am. Chem. Soc. 2021, 143, } \\
\text { 3509-3518 }\end{array}$ \\
\hline 10 & {$\left[\mathrm{Zn}_{4}\left(\mu_{4}-\mathrm{H}_{2} \mathrm{O}\right)(\mathrm{TBSC})\right]\left[\mathrm{L}_{1}\right]_{2}$} & isopropanol & J. Am. Chem. Soc. 2019, 141, \\
\hline 11 & {$\left[\mathrm{Zn}_{4}\left(\mu_{4}-\mathrm{H}_{2} \mathrm{O}\right)(\mathrm{TBSC})\right]\left[\mathrm{L}_{2}\right]_{2}$} & $\mathrm{MeCN}$ & $17685-17695$ \\
\hline 12 & LMOF 1 & 1,4-dioxane or isopropyl ether & Our work \\
\hline
\end{tabular}




\section{NMR}

18.1 Synthesis of 2,3-dihydroquinazolinone

2-phenyl-2,3-dihydroquinazolin-4(1H)-one

${ }^{1} \mathrm{H}$ NMR $\left(400 \mathrm{MHz}, d_{6}\right.$-DMSO) $\delta 8.28(\mathrm{~s}, 1 \mathrm{H}), 7.60(\mathrm{~d}, \mathrm{~J}=8.0 \mathrm{~Hz}, 1 \mathrm{H}), 7.47(\mathrm{~d}, \mathrm{~J}=7.2 \mathrm{~Hz}, 2 \mathrm{H})$, $7.41 \sim 7.34(\mathrm{~m}, 3 \mathrm{H}), 7.22(\mathrm{t}, \mathrm{J}=8.0 \mathrm{~Hz}, 1 \mathrm{H}), 7.10(\mathrm{~s}, 1 \mathrm{H}), 6.74(\mathrm{~d}, \mathrm{~J}=8 \mathrm{~Hz}, 1 \mathrm{H}), 6.65(\mathrm{t}, \mathrm{J}=8.0 \mathrm{~Hz}$, $1 \mathrm{H}), 5.75(\mathrm{~s}, 1 \mathrm{H})$.
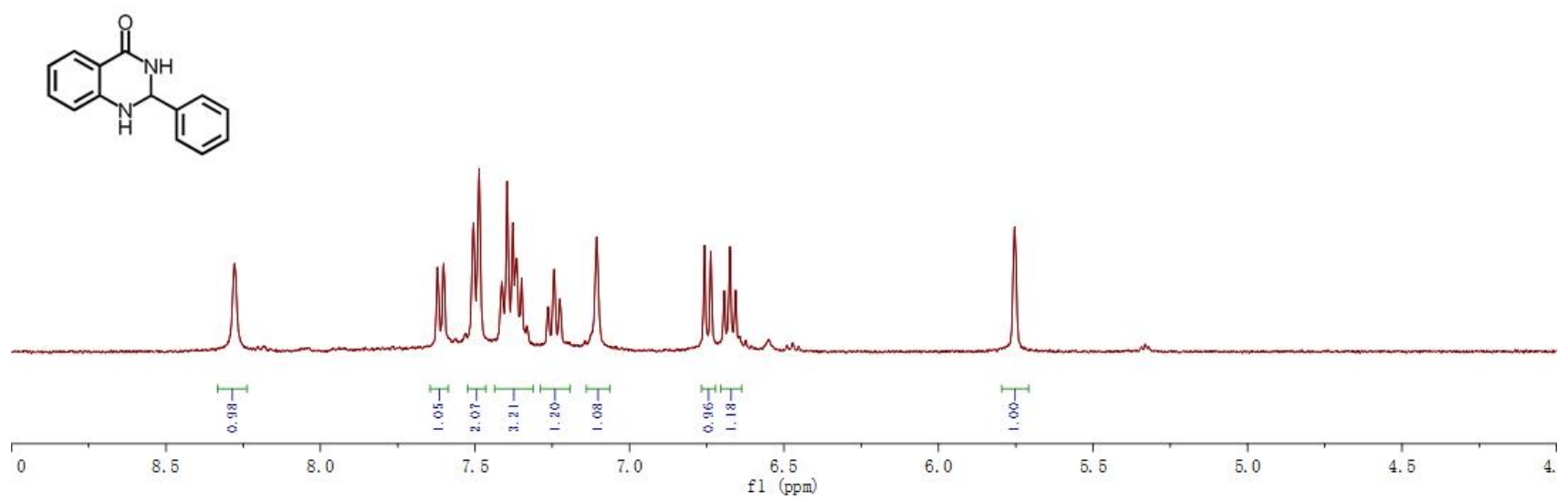

2-(3-fluorophenyl)-2,3-dihydroquinazolin-4(1H)-one

${ }^{1} \mathrm{H}$ NMR $\left(400 \mathrm{MHz} \mathrm{CDCl}_{3}\right) \delta 7.87(\mathrm{~d}, \mathrm{~J}=8.0 \mathrm{~Hz}, 1 \mathrm{H}), 7.33(\mathrm{~m}, 1 \mathrm{H}), 7.26(\mathrm{~m}, 3 \mathrm{H}), 7.06(\mathrm{t}, \mathrm{J}=8.0$ $\mathrm{Hz}, 1 \mathrm{H}), 6.83(\mathrm{t}, \mathrm{J}=8 \mathrm{~Hz}, 1 \mathrm{H}), 6.60(\mathrm{~d}, \mathrm{~J}=8.0 \mathrm{~Hz}, 1 \mathrm{H}), 5.84(\mathrm{~s}, 1 \mathrm{H}), 5.75(\mathrm{~s}, 1 \mathrm{H}), 4.32(\mathrm{~s}, 1 \mathrm{H})$.

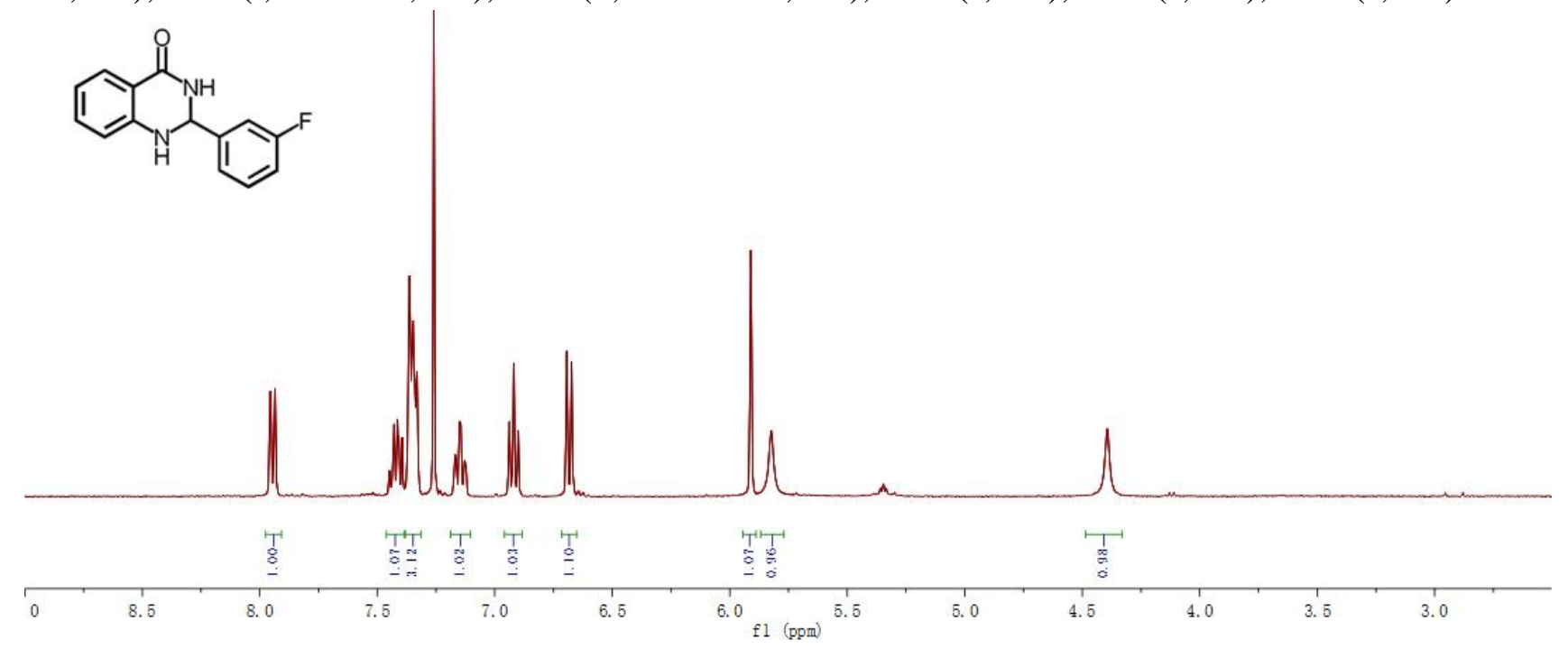

\section{2-(4-fluorophenyl)-2,3-dihydroquinazolin-4(1H)-one}

${ }^{1} \mathrm{H}$ NMR (400 MHz, $\left.\mathrm{CDCl}_{3}\right) \delta 7.93(\mathrm{~d}, \mathrm{~J}=8.0 \mathrm{~Hz}, 1 \mathrm{H}), 7.58(\mathrm{~m}, 2 \mathrm{H}), 7.26(\mathrm{~m}, 3 \mathrm{H}), 7.32(\mathrm{t}, \mathrm{J}=8.0$ $\mathrm{Hz}, 1 \mathrm{H}), 7.11(\mathrm{t}, \mathrm{J}=8 \mathrm{~Hz}, 2 \mathrm{H}), 6.89(\mathrm{t}, \mathrm{J}=8.0 \mathrm{~Hz}, 1 \mathrm{H}), 6.67(\mathrm{~d}, \mathrm{~J}=8 \mathrm{~Hz}, 1 \mathrm{H}), 5.90(\mathrm{~s}, 1 \mathrm{H}), 5.80(\mathrm{~s}$, $1 \mathrm{H}), 4.37(\mathrm{~s}, 1 \mathrm{H})$. 


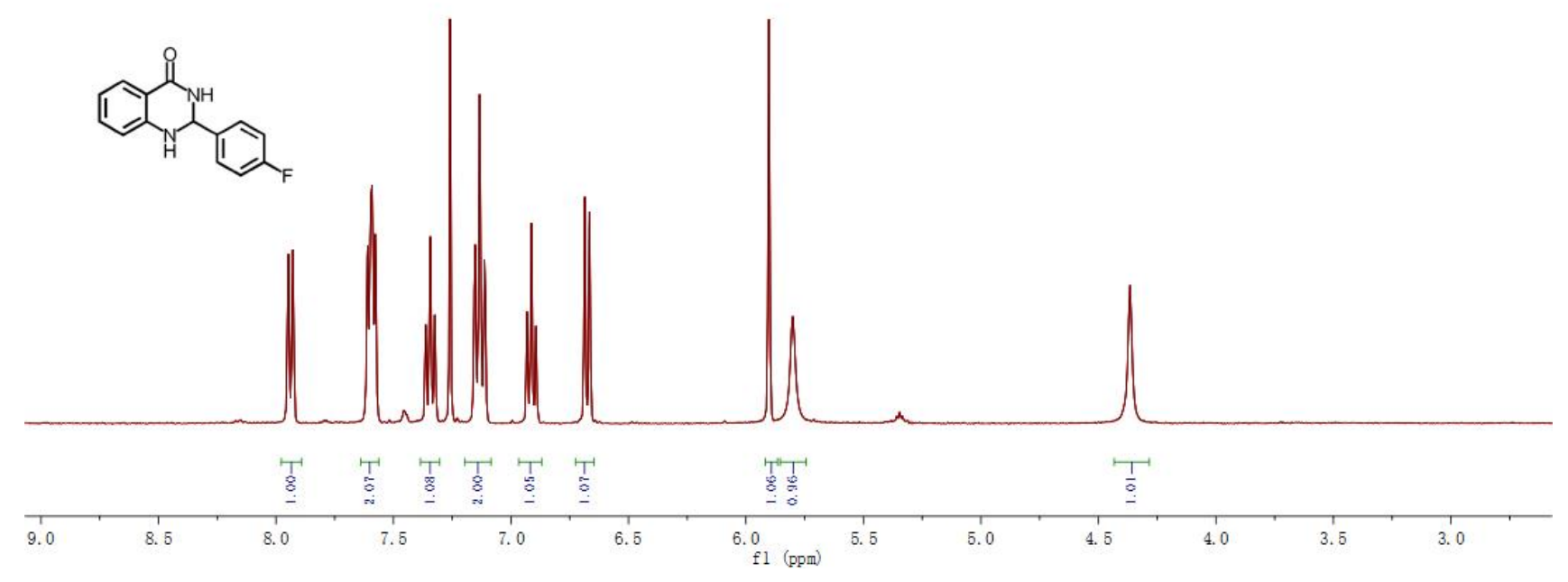

2-(2-chlorophenyl)-2,3-dihydroquinazolin-4(1H)-one

${ }^{1} \mathrm{H}$ NMR (400 MHz, $\left.\mathrm{CDCl}_{3}\right) \delta 7.93(\mathrm{~d}, \mathrm{~J}=8.0 \mathrm{~Hz}, 1 \mathrm{H}), 7.74(\mathrm{t}, \mathrm{J}=4 \mathrm{~Hz}, 1 \mathrm{H}), 7.40(\mathrm{~m}, 1 \mathrm{H}), 7.26(\mathrm{~m}$, $3 \mathrm{H}), 6.87(\mathrm{t}, \mathrm{J}=8 \mathrm{~Hz}, 1 \mathrm{H}), 6.66(\mathrm{~d}, \mathrm{~J}=8.0 \mathrm{~Hz}, 1 \mathrm{H}), 6.36(\mathrm{~s}, 1 \mathrm{H}), 5.96(\mathrm{~s}, 1 \mathrm{H}), 4.60(\mathrm{~s}, 1 \mathrm{H})$.

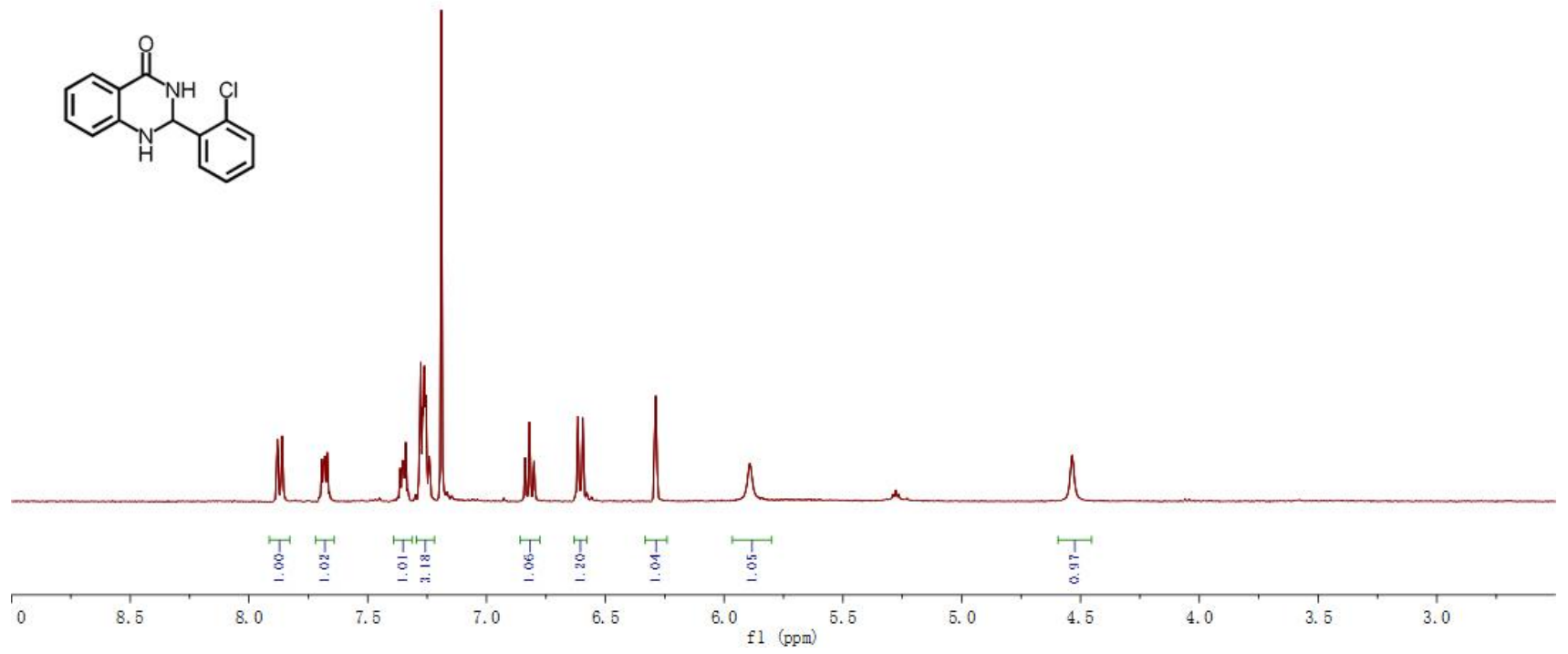

2-(4-chlorophenyl)-2,3-dihydroquinazolin-4(1H)-one

${ }^{1} \mathrm{H}$ NMR (400 MHz, $\left.\mathrm{CDCl}_{3}\right) \delta 7.93(\mathrm{~d}, \mathrm{~J}=8.0 \mathrm{~Hz}, 1 \mathrm{H}), 7.53(\mathrm{~d}, \mathrm{~J}=8 \mathrm{~Hz}, 2 \mathrm{H}), 7.37(\mathrm{~d}, \mathrm{~J}=8 \mathrm{~Hz}, 2 \mathrm{H})$,

$7.33(\mathrm{t}, \mathrm{J}=8.0 \mathrm{~Hz}, 1 \mathrm{H}), 6.90(\mathrm{t}, \mathrm{J}=8 \mathrm{~Hz}, 1 \mathrm{H}), 6.67(\mathrm{~d}, \mathrm{~J}=8 \mathrm{~Hz}, 1 \mathrm{H}), 5.90(\mathrm{~s}, 1 \mathrm{H}), 5.80(\mathrm{~s}, 1 \mathrm{H}), 4.36$ (s, 1H). 


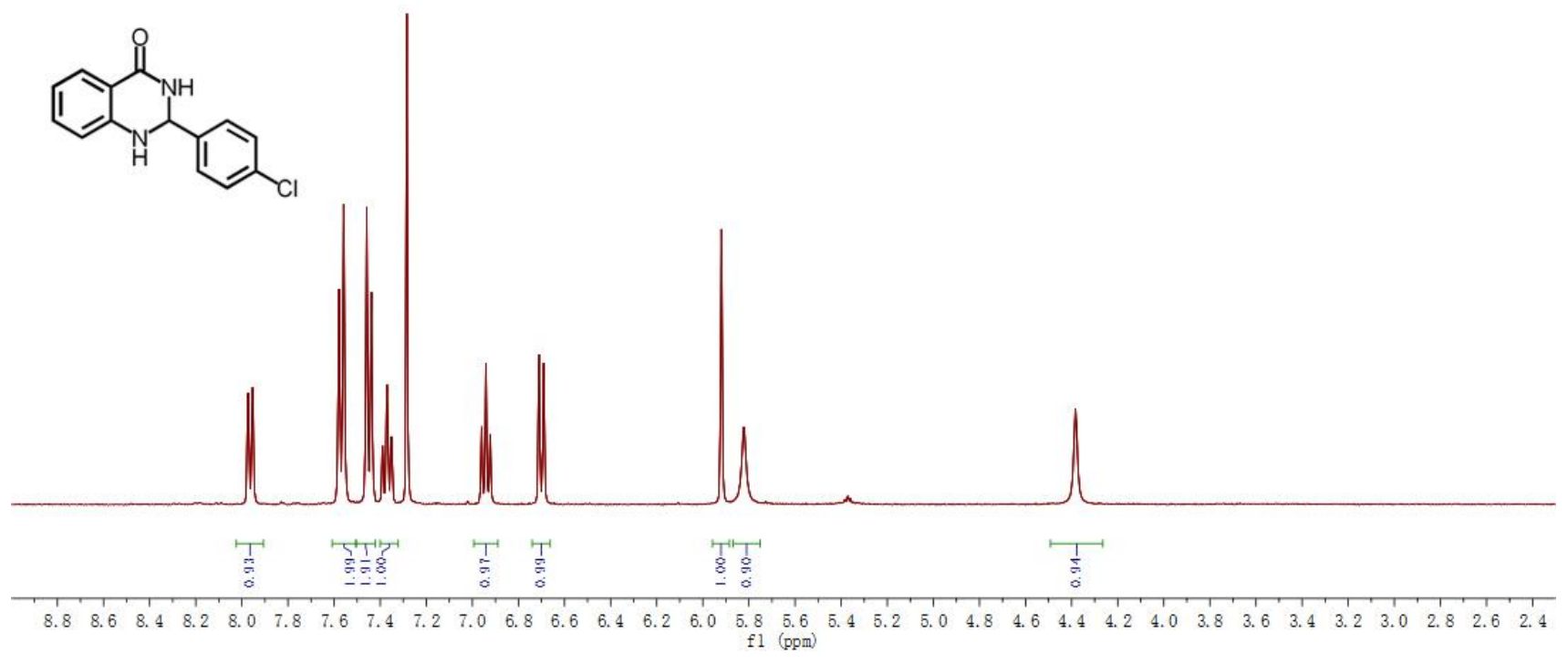

\section{2-(2-bromophenyl)-2,3-dihydroquinazolin-4(1H)-one}

${ }^{1} \mathrm{H}$ NMR (400 MHz, $\left.\mathrm{CDCl}_{3}\right) \delta 7.93(\mathrm{~d}, \mathrm{~J}=8.0 \mathrm{~Hz}, 1 \mathrm{H}), 7.75(\mathrm{~d}, \mathrm{~J}=8 \mathrm{~Hz}, 1 \mathrm{H}), 7.59$ (d, J = $\left.8 \mathrm{~Hz}, 1 \mathrm{H}\right)$, $7.38(\mathrm{t}, \mathrm{J}=8.0 \mathrm{~Hz}, 1 \mathrm{H}), 7.33(\mathrm{t}, \mathrm{J}=8 \mathrm{~Hz}, 1 \mathrm{H}), 7.26(\mathrm{t}, \mathrm{J}=8 \mathrm{~Hz}, 1 \mathrm{H}), 6.87(\mathrm{t}, \mathrm{J}=8 \mathrm{~Hz}, 1 \mathrm{H}), 6.67(\mathrm{~d}, \mathrm{~J}$ $=8 \mathrm{~Hz}, 1 \mathrm{H}), 6.31(\mathrm{~s}, 1 \mathrm{H}), 5.96(\mathrm{~s}, 1 \mathrm{H}), 4.36(\mathrm{~s}, 1 \mathrm{H})$.

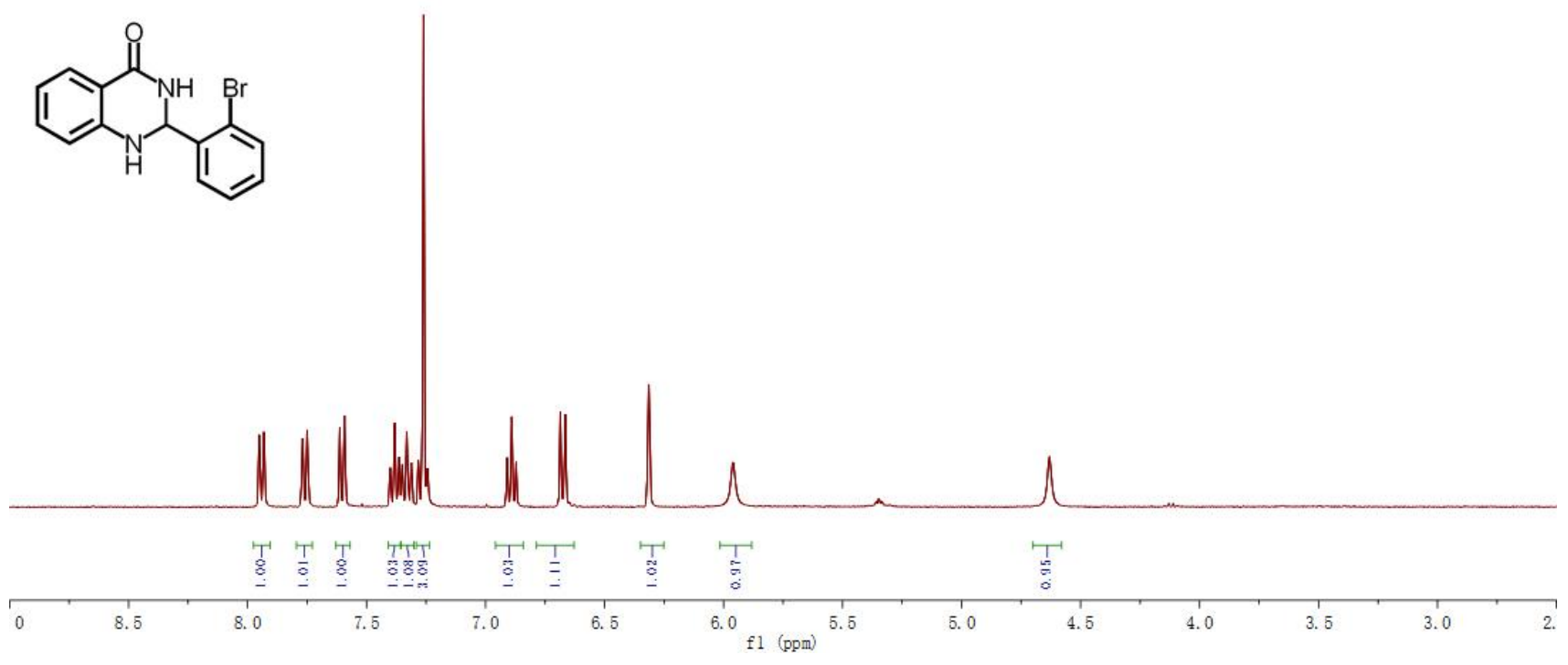

2-(4-bromophenyl)-2,3-dihydroquinazolin-4(1H)-one

${ }^{1} \mathrm{H}$ NMR (400 MHz, CDCl 3$) \delta 7.94(\mathrm{~d}, \mathrm{~J}=8.0 \mathrm{~Hz}, 1 \mathrm{H}), 7.58(\mathrm{~d}, \mathrm{~J}=8 \mathrm{~Hz}, 2 \mathrm{H}), 7.47$ (d, J = 8 Hz, 2H), $7.33(\mathrm{t}, \mathrm{J}=8.0 \mathrm{~Hz}, 1 \mathrm{H}), 6.90(\mathrm{t}, \mathrm{J}=8 \mathrm{~Hz}, 1 \mathrm{H}), 6.67(\mathrm{~d}, \mathrm{~J}=8 \mathrm{~Hz}, 1 \mathrm{H}), 5.89(\mathrm{~s}, 1 \mathrm{H}), 5.71(\mathrm{~s}, 1 \mathrm{H}), 4.34$ $(\mathrm{s}, 1 \mathrm{H})$. 


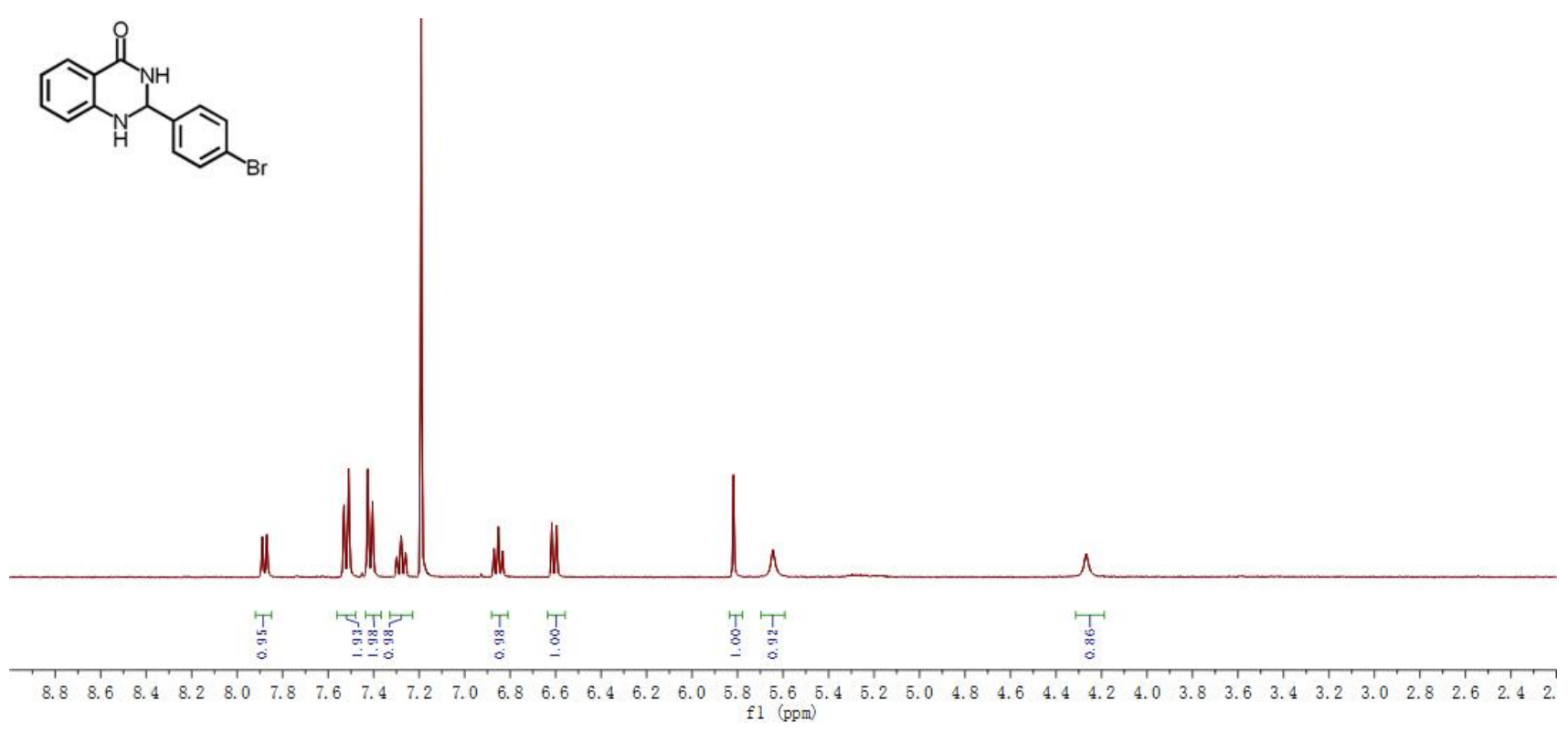

2-(2-methoxyphenyl)-2,3-dihydroquinazolin-4(1H)-one

${ }^{1} \mathrm{H}$ NMR (400 MHz, $\left.\mathrm{CDCl}_{3}\right) \delta 7.89(\mathrm{~d}, \mathrm{~J}=8.0 \mathrm{~Hz}, 1 \mathrm{H}), 7.53(\mathrm{~d}, \mathrm{~J}=8 \mathrm{~Hz}, 1 \mathrm{H}), 7.31(\mathrm{~m}, 2 \mathrm{H}), 6.98(\mathrm{t}$, $\mathrm{J}=4.0 \mathrm{~Hz}, 1 \mathrm{H}), 6.91(\mathrm{~d}, \mathrm{~J}=8 \mathrm{~Hz}, 1 \mathrm{H}), 6.83(\mathrm{t}, \mathrm{J}=8.0 \mathrm{~Hz}, 1 \mathrm{H}), 6.64(\mathrm{~d}, \mathrm{~J}=8 \mathrm{~Hz}, 1 \mathrm{H}) 6.24(\mathrm{~s}, 1 \mathrm{H})$, $6.14(\mathrm{~s}, 1 \mathrm{H}), 4.68(\mathrm{~s}, 1 \mathrm{H}), 3.88(\mathrm{~s}, 3 \mathrm{H})$.
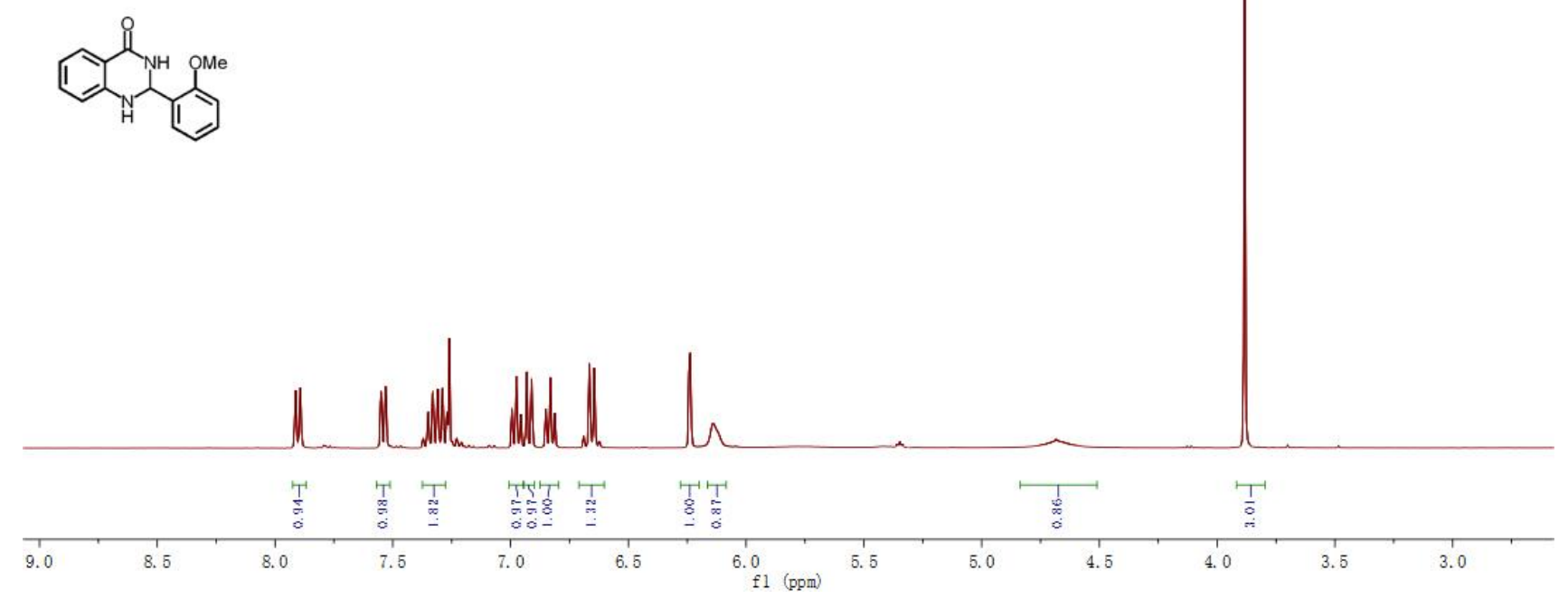

\section{2-(o-tolyl)-2,3-dihydroquinazolin-4(1H)-one}

${ }^{1} \mathrm{H} \mathrm{NMR}\left(400 \mathrm{MHz}, \mathrm{CDCl}_{3}\right) \delta 7.96(\mathrm{~d}, \mathrm{~J}=8.0 \mathrm{~Hz}, 1 \mathrm{H}), 7.72(\mathrm{~d}, \mathrm{~J}=8 \mathrm{~Hz}, 1 \mathrm{H}), 7.34(\mathrm{~m}, 3 \mathrm{H}), 7.25(\mathrm{t}, \mathrm{J}$ $=8.0 \mathrm{~Hz}, 1 \mathrm{H}), 6.92(\mathrm{t}, \mathrm{J}=8 \mathrm{~Hz}, 1 \mathrm{H}), 6.68(\mathrm{~d}, \mathrm{~J}=8.0 \mathrm{~Hz}, 1 \mathrm{H}), 6.17(\mathrm{~s}, 1 \mathrm{H}), 5.69(\mathrm{~s}, 1 \mathrm{H}), 4.32(\mathrm{~s}, 1 \mathrm{H})$, $2.48(\mathrm{~s}, 3 \mathrm{H})$. 


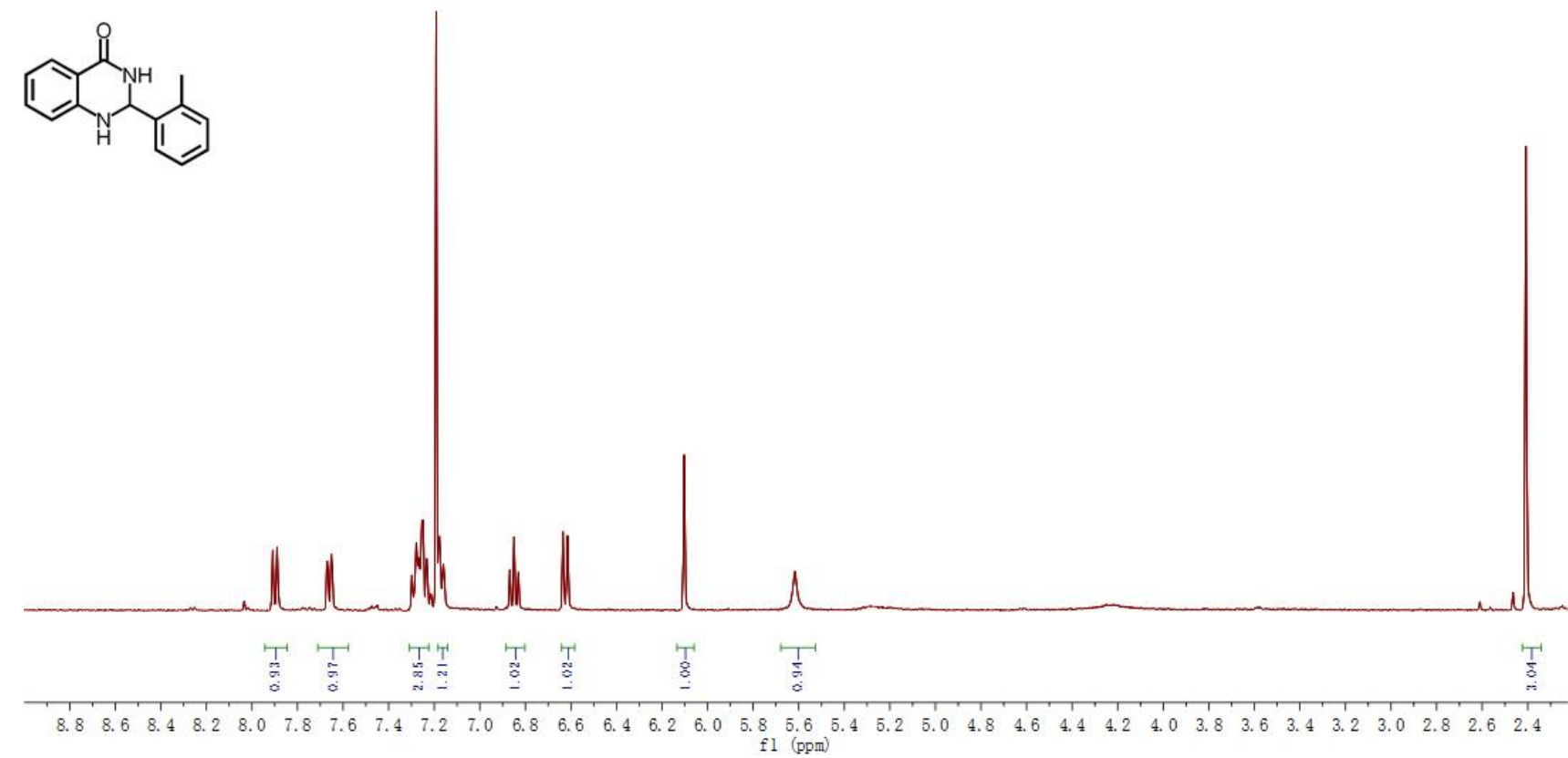

\section{2-(4-ethylphenyl)-2,3-dihydroquinazolin-4(1H)-one}

${ }^{1} \mathrm{H}$ NMR (400 MHz, $\left.\mathrm{CDCl}_{3}\right) \delta 7.94(\mathrm{~d}, \mathrm{~J}=8.0 \mathrm{~Hz}, 1 \mathrm{H}), 7.50(\mathrm{~d}, \mathrm{~J}=8 \mathrm{~Hz}, 2 \mathrm{H}), 7.33(\mathrm{t}, \mathrm{J}=8 \mathrm{~Hz}, 1 \mathrm{H})$, $7.29(\mathrm{~d}, \mathrm{~J}=8.0 \mathrm{~Hz}, 2 \mathrm{H}), 6.88(\mathrm{t}, \mathrm{J}=8 \mathrm{~Hz}, 1 \mathrm{H}), 6.67(\mathrm{~d}, \mathrm{~J}=8.0 \mathrm{~Hz}, 1 \mathrm{H}), 5.88(\mathrm{~s}, 1 \mathrm{H}), 5.73(\mathrm{~s}, 1 \mathrm{H})$, $4.35(\mathrm{~s}, 1 \mathrm{H}), 2.67(\mathrm{dd}, \mathrm{J}=8 \mathrm{~Hz}, 2 \mathrm{H}), 1.26(\mathrm{t}, \mathrm{J}=8 \mathrm{~Hz}, 3 \mathrm{H})$.

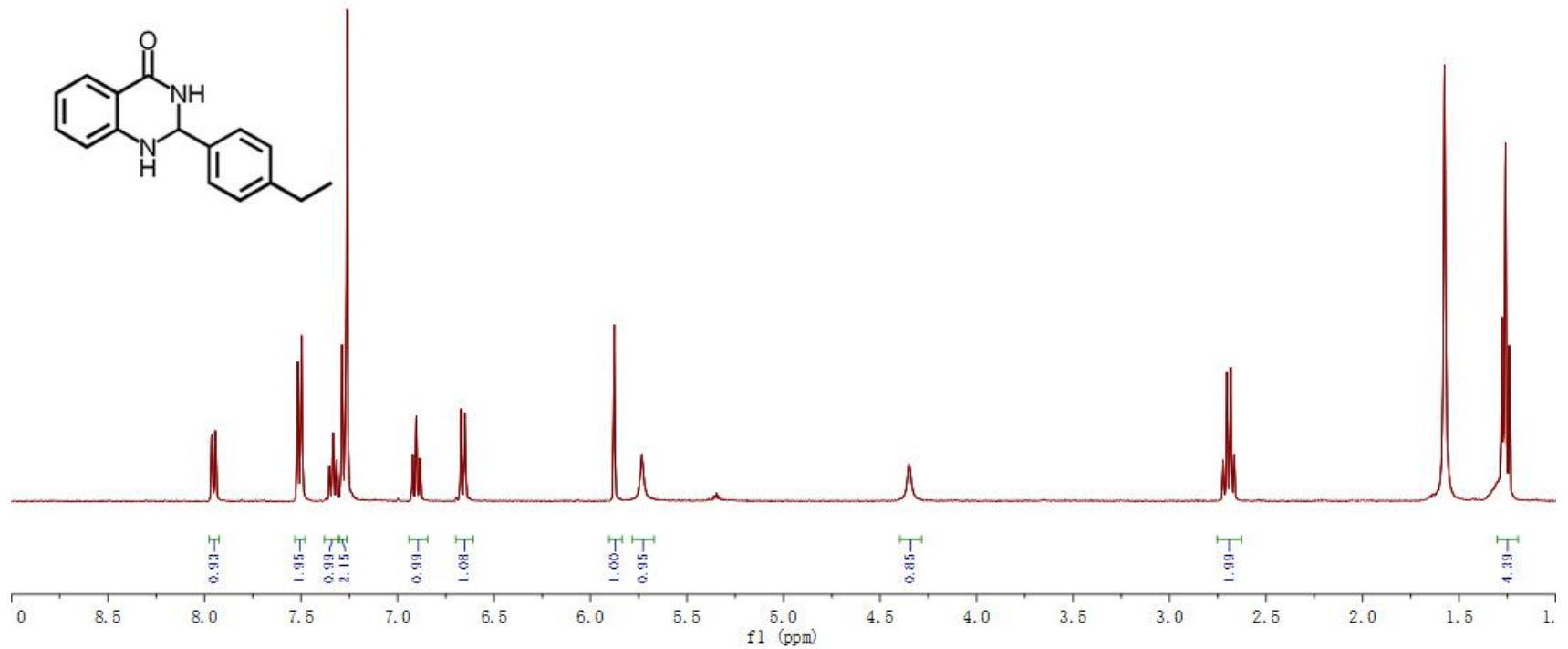

\section{2-(naphthalen-1-yl)-2,3-dihydroquinazolin-4(1H)-one}

${ }^{1} \mathrm{H}$ NMR $\left(400 \mathrm{MHz}, \mathrm{CDCl}_{3}\right) \delta 8.45(\mathrm{~d}, \mathrm{~J}=4.0 \mathrm{~Hz}, 1 \mathrm{H}), 7.98(\mathrm{~d}, \mathrm{~J}=8 \mathrm{~Hz}, 1 \mathrm{H}), 7.91(\mathrm{~d}, \mathrm{~J}=8 \mathrm{~Hz}, 2 \mathrm{H})$, $7.78(\mathrm{~d}, \mathrm{~J}=8 \mathrm{~Hz}, 1 \mathrm{H}), 7.50-7.56(\mathrm{~m}, 3 \mathrm{H}), 7.34(\mathrm{t}, \mathrm{J}=8.0 \mathrm{~Hz}, 1 \mathrm{H}), 6.91(\mathrm{t}, \mathrm{J}=8 \mathrm{~Hz}, 1 \mathrm{H}), 6.69(\mathrm{~d}, \mathrm{~J}=$ $8 \mathrm{~Hz}, 1 \mathrm{H}) 6.57(\mathrm{~s}, 1 \mathrm{H}), 5.95(\mathrm{~s}, 1 \mathrm{H}), 4.60(\mathrm{~s}, 1 \mathrm{H})$. 


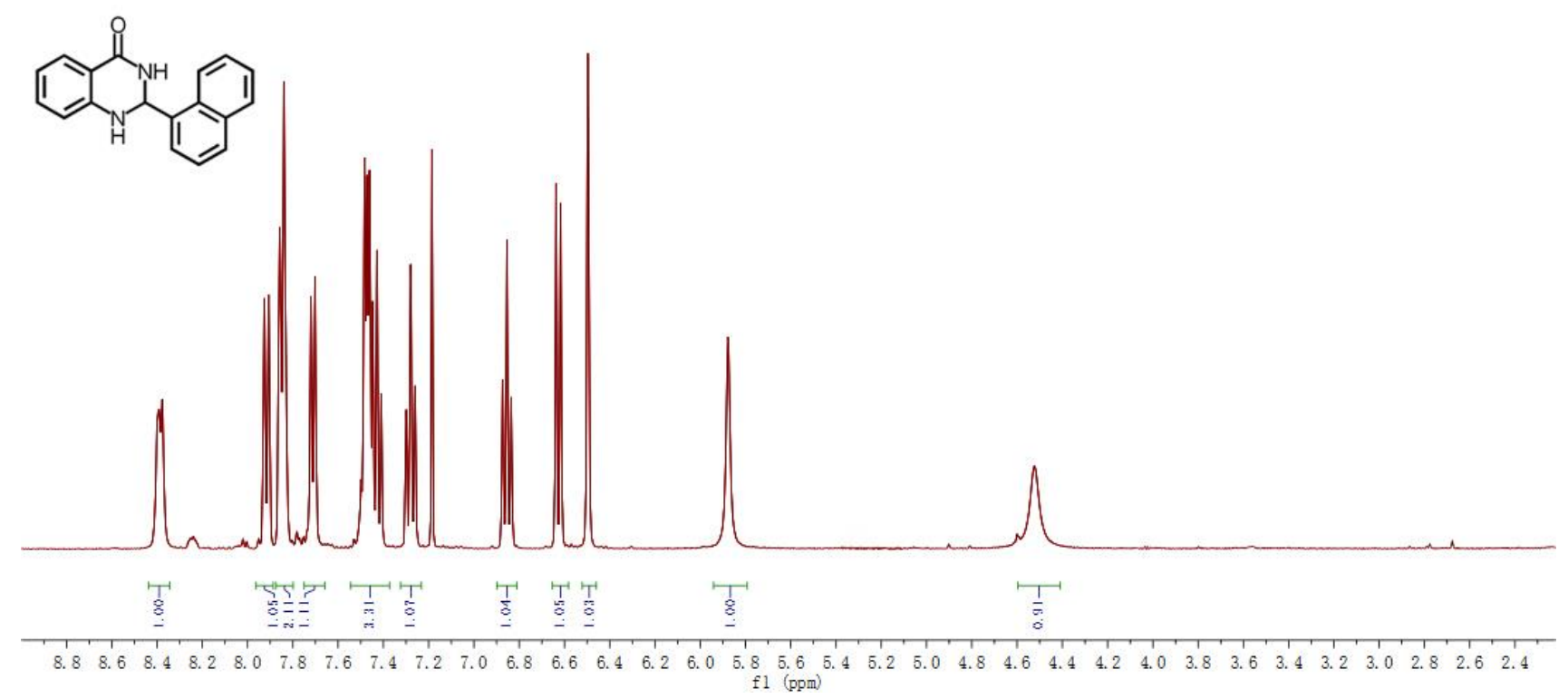

\section{2-(anthracen-9-yl)-2,3-dihydroquinazolin-4(1H)-one}

${ }^{1} \mathrm{H}$ NMR (400 MHz, $d_{6}$-DMSO) $\delta 9.77(\mathrm{~s}, 1 \mathrm{H}), 8.86(\mathrm{~m}, 3 \mathrm{H}), 8.20(\mathrm{~d}, \mathrm{~J}=8 \mathrm{~Hz}, 2 \mathrm{H}), 8.13$ (br, 1H), $7.85(\mathrm{dd}, \mathrm{J}=8 \mathrm{~Hz}, 4 \mathrm{~Hz}, 1 \mathrm{H}), 7.60-7.68(\mathrm{~m}, 5 \mathrm{H}), 7.54(\mathrm{br}, 1 \mathrm{H}), 7.53(\mathrm{~d}, \mathrm{~J}=4 \mathrm{~Hz}, 1 \mathrm{H}), 7.42(\mathrm{td}, \mathrm{J}=8$ $\mathrm{Hz}, 2.4 \mathrm{~Hz}, 1 \mathrm{H})$.

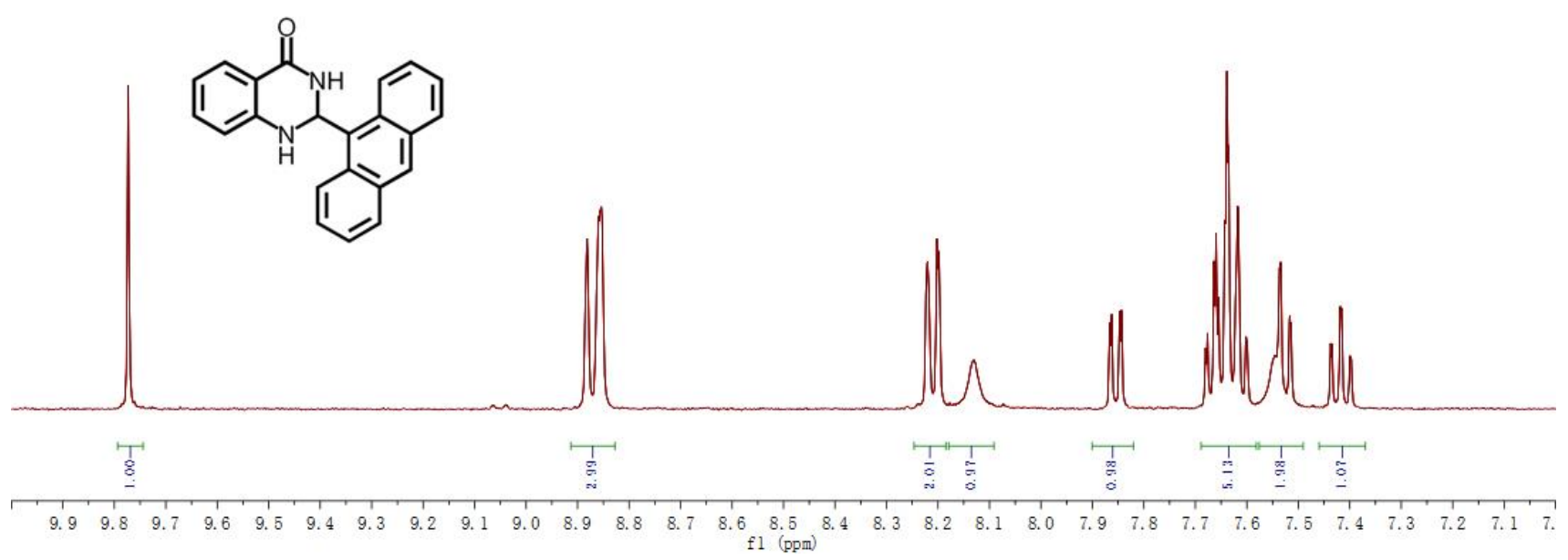

\section{2-(pyren-1-yl)-2,3-dihydroquinazolin-4(1H)-one}

${ }^{1} \mathrm{H}$ NMR (400 MHz, d6-DMSO) $\delta 9.64(\mathrm{~s}, 1 \mathrm{H}), 9.24(\mathrm{~d}, \mathrm{~J}=12 \mathrm{~Hz}, 1 \mathrm{H}), 8.79(\mathrm{~d}, \mathrm{~J}=8 \mathrm{~Hz}, 1 \mathrm{H}), 8.46-$ $8.29(\mathrm{~m}, 7 \mathrm{H}), 8.18(\mathrm{t}, \mathrm{J}=8 \mathrm{~Hz}, 1 \mathrm{H}), 7.94(\mathrm{dd}, \mathrm{J}=8 \mathrm{~Hz}, 4 \mathrm{~Hz}, 1 \mathrm{H}), 7.68$ (br, 1H), 7.63 (td, J = $8 \mathrm{~Hz}$, $2.4 \mathrm{~Hz}, 1 \mathrm{H}), 7.50(\mathrm{~d}, \mathrm{~J}=4 \mathrm{~Hz}, 1 \mathrm{H}), 7.41(\mathrm{td}, \mathrm{J}=8 \mathrm{~Hz}, 2.4 \mathrm{~Hz}, 1 \mathrm{H})$. 


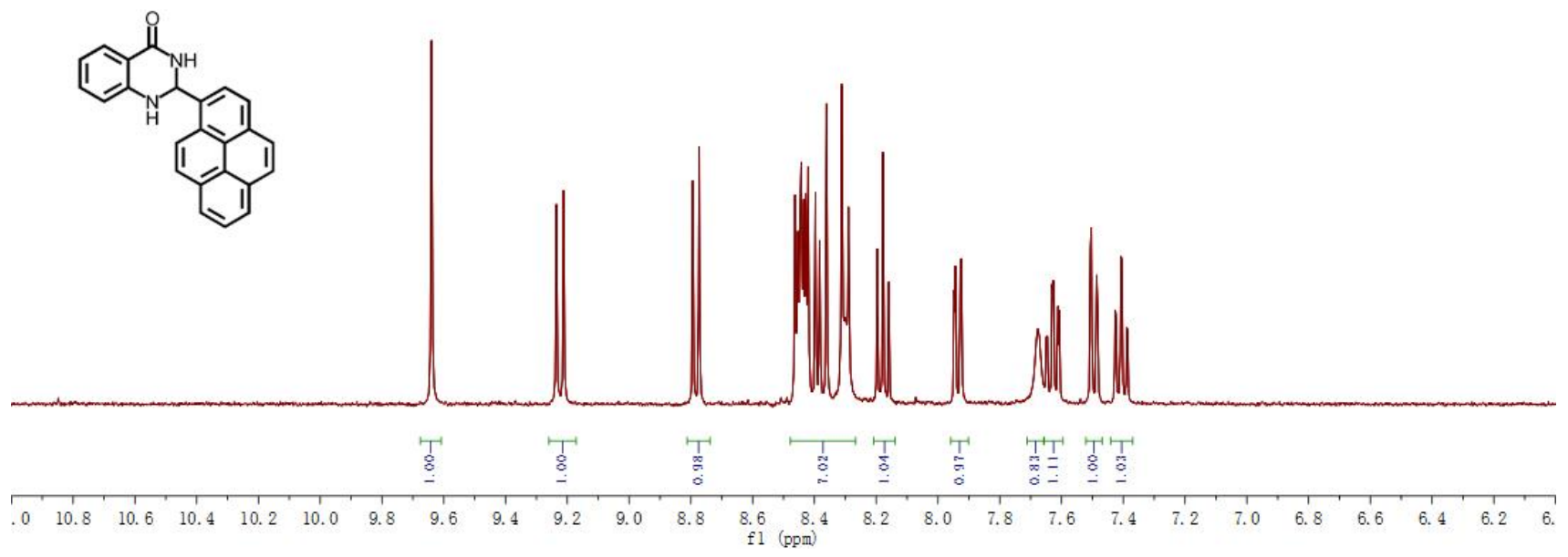

2-(4-(anthracen-9-yl)phenyl)-2,3-dihydroquinazolin-4(1H)-one

${ }^{1} \mathrm{H}$ NMR (400 MHz, d6-DMSO) $\delta 8.69(\mathrm{~s}, 1 \mathrm{H}), 8.44(\mathrm{~s}, 1 \mathrm{H}), 8.18(\mathrm{~d}, \mathrm{~J}=12 \mathrm{~Hz}, 2 \mathrm{H}), 7.77$ (d, J = $8 \mathrm{~Hz}$, $2 \mathrm{H}), 7.67(\mathrm{~d}, \mathrm{~J}=8 \mathrm{~Hz}, 1 \mathrm{H}), 7.54-7.52(\mathrm{~m}, 4 \mathrm{H}), 7.46-7.44(\mathrm{~m}, 4 \mathrm{H}), 7.32-7.28(\mathrm{~m}, 2 \mathrm{H}), 6.87(\mathrm{~d}, \mathrm{~J}=8$ $\mathrm{Hz}, 1 \mathrm{H}), 6.71(\mathrm{t}, \mathrm{J}=8 \mathrm{~Hz}, 1 \mathrm{H}), 5.96(\mathrm{~s}, 1 \mathrm{H})$.

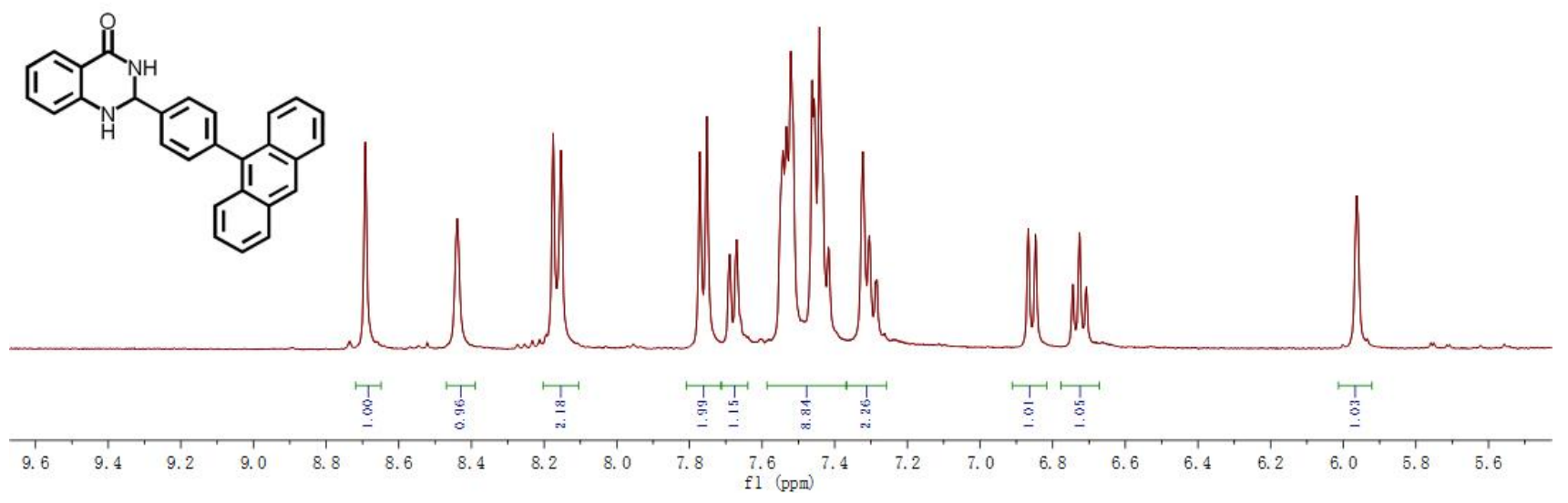

2-(3,5-bis((3,5-di-tert-butylbenzyl)oxy)phenyl)-2,3-dihydroquinazolin-4(1H)-one

${ }^{1} \mathrm{H}$ NMR (400 MHz, d6-DMSO) $\delta 12.51(\mathrm{~s}, 1 \mathrm{H}), 8.16(\mathrm{~d}, \mathrm{~J}=12 \mathrm{~Hz}, 2 \mathrm{H}), 7.48(\mathrm{td}, \mathrm{J}=8 \mathrm{~Hz}, 4 \mathrm{~Hz}, 1 \mathrm{H})$, $7.74(\mathrm{~d}, \mathrm{~J}=8 \mathrm{~Hz}, 1 \mathrm{H}), 7.56-7.52(\mathrm{~m}, 3 \mathrm{H}), 7.38(\mathrm{~s}, 2 \mathrm{H}), 7.34(\mathrm{~m}, 4 \mathrm{H}), 6.93(\mathrm{~s}, 1 \mathrm{H}), 5.76(\mathrm{~s}, 1 \mathrm{H}), 5.17$ $(\mathrm{s}, 4 \mathrm{H}), 1.31(\mathrm{~s}, 36 \mathrm{H})$. 


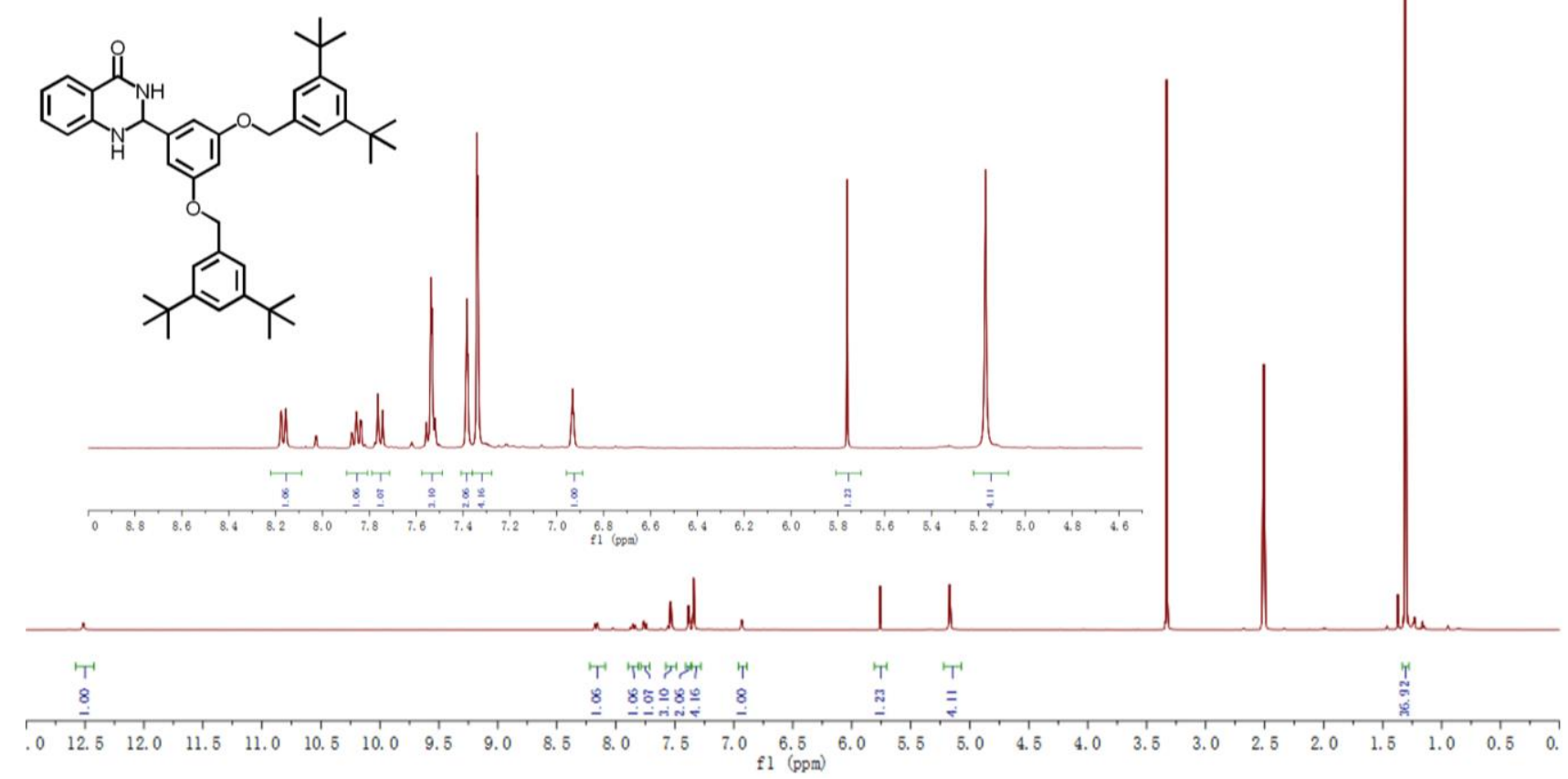

\subsection{Synthesis of propargylamines}

\section{4-(1,3-diphenylprop-2-yn-1-yl)morpholine}

${ }^{1} \mathrm{H}$ NMR $\left(400 \mathrm{MHz}, \mathrm{CDCl}_{3}\right) \delta 7.63(\mathrm{~d}, \mathrm{~J}=8.0 \mathrm{~Hz}, 2 \mathrm{H}), 7.54-7.51(\mathrm{~m}, 2 \mathrm{H}), 7.40-7.31(\mathrm{~m}, 6 \mathrm{H}), 4.80$ (s, $1 \mathrm{H}), 3.76-3.72(\mathrm{~m}, 4 \mathrm{H}), 2.65-2.63(\mathrm{~m}, 4 \mathrm{H})$.
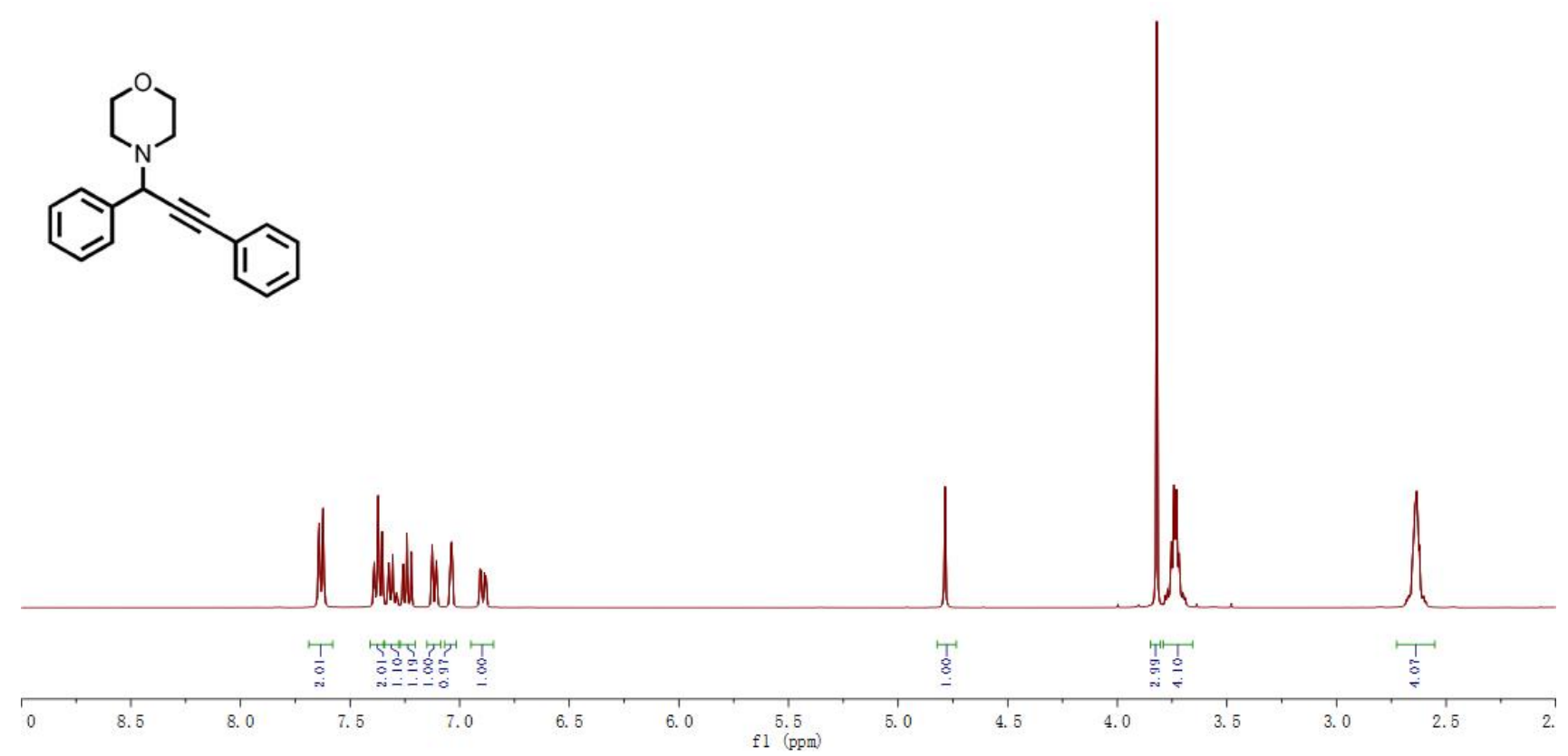

4-(1-(3-fluorophenyl)-3-phenylprop-2-yn-1-yl)morpholine

${ }^{1} \mathrm{H}$ NMR $\left(400 \mathrm{MHz}, \mathrm{CDCl}_{3}\right) \delta$ 7.53-7.50 (m, 2H), $7.43(\mathrm{~d}, \mathrm{~J}=8 \mathrm{~Hz}, 1 \mathrm{H}), 7.37(\mathrm{~d}, \mathrm{~J}=8 \mathrm{~Hz}, 1 \mathrm{H}), 7.40-$ $7.30(\mathrm{~m}, 4 \mathrm{H}), 7.02-6.98(\mathrm{~m}, 1 \mathrm{H}), 4.79(\mathrm{~s}, 1 \mathrm{H}), 3.76-3.74(\mathrm{~m}, 4 \mathrm{H}), 2.64-2.62(\mathrm{~m}, 4 \mathrm{H})$. 


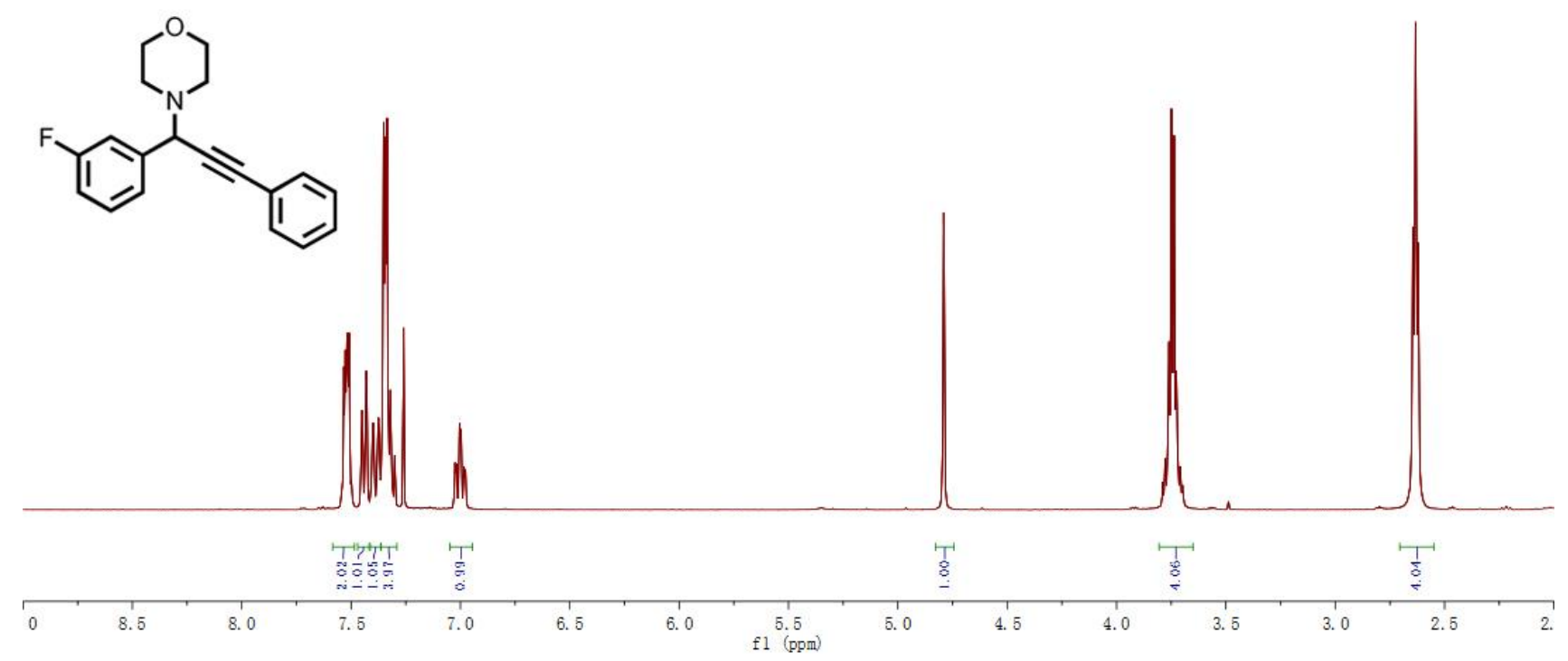

4-(1-(4-chlorophenyl)-3-phenylprop-2-yn-1-yl)morpholine

${ }^{1} \mathrm{H}$ NMR $\left(400 \mathrm{MHz}, \mathrm{CDCl}_{3}\right) \delta$ 7.59-7.57 (d, J = $\left.8 \mathrm{~Hz}, 2 \mathrm{H}\right), 7.52-7.50(\mathrm{~m}, 2 \mathrm{H}), 7.33-7.35(\mathrm{~m}, 5 \mathrm{H})$, $4.76(\mathrm{~s}, 1 \mathrm{H}), 3.75-3.71(\mathrm{~m}, 4 \mathrm{H}), 2.63-2.60(\mathrm{~m}, 4 \mathrm{H})$.

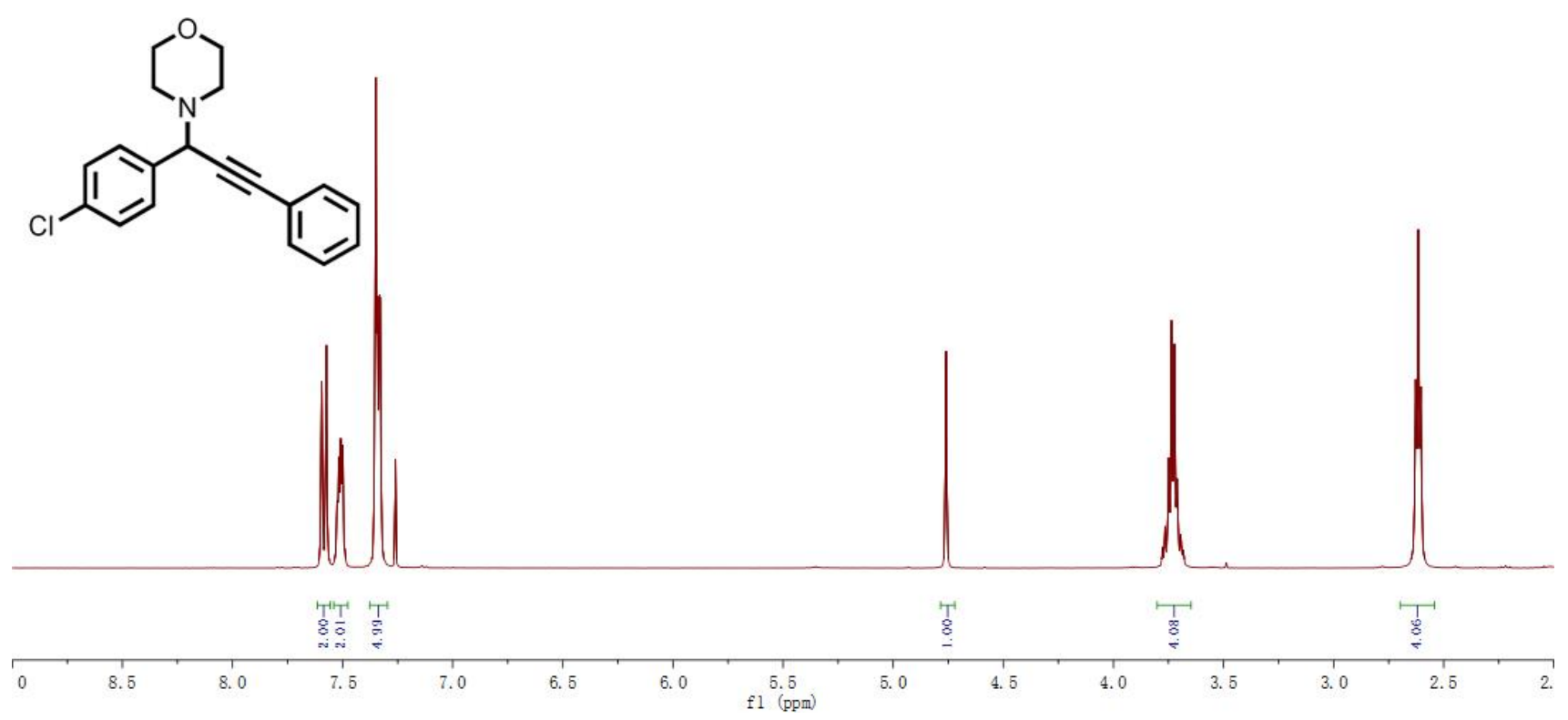

4-(1-(4-bromophenyl)-3-phenylprop-2-yn-1-yl)morpholine

${ }^{1} \mathrm{H}$ NMR (400 MHz, $\left.\mathrm{CDCl}_{3}\right) \delta$ 7.54-7.48 (m, 6H), 7.36-7.33 (m, 3H), $4.74(\mathrm{~s}, 1 \mathrm{H}), 3.76-3.71(\mathrm{~m}$, $4 \mathrm{H}), 2.62-2.60(\mathrm{~m}, 4 \mathrm{H})$. 


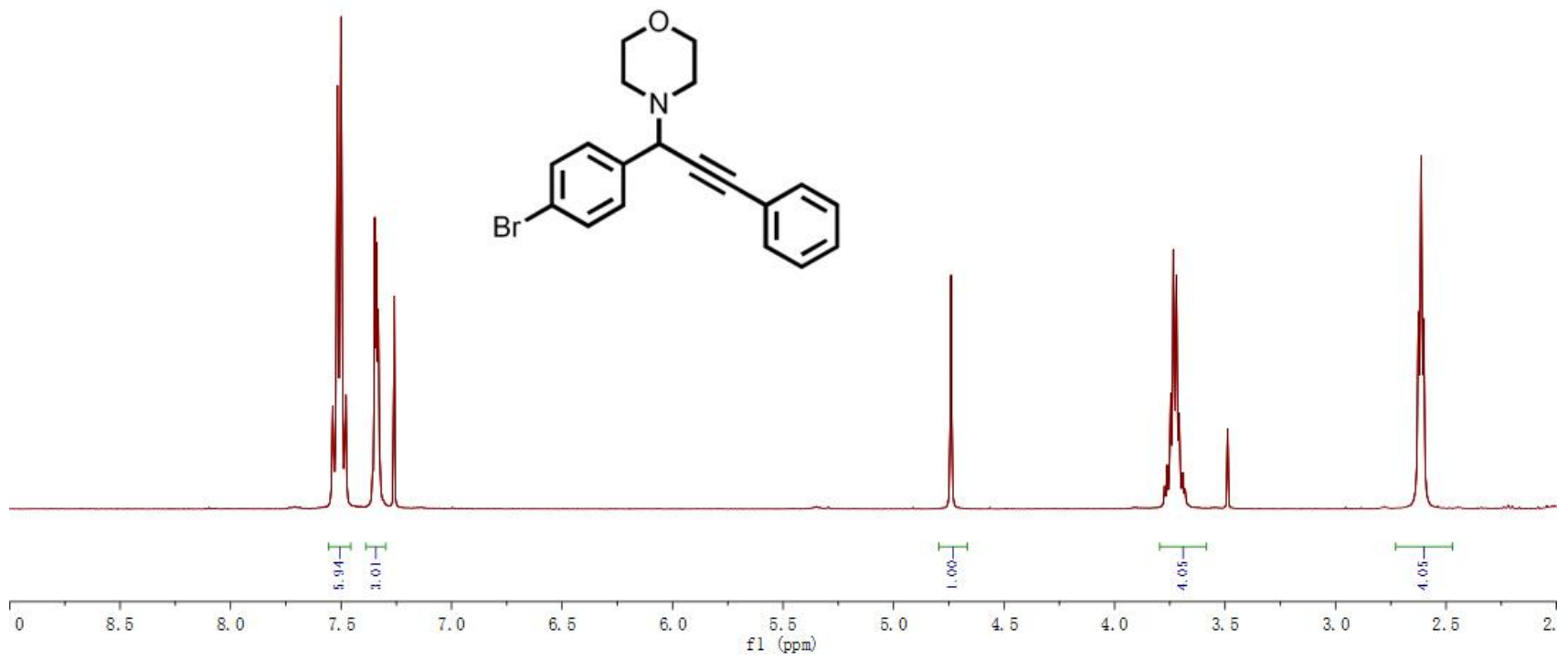

4-(1-(2-methoxyphenyl)-3-phenylprop-2-yn-1-yl)morpholine

${ }^{1} \mathrm{H}$ NMR (400 MHz, $\mathrm{CDCl}_{3}$ ) $\delta$ 7.65-7.63 (dd, J=8 Hz, $\left.1.6 \mathrm{~Hz}, 1 \mathrm{H}\right), 7.48-7.46$ (m, 2H), 7.31-7.27 (m, $4 \mathrm{H}), 7.00-6.97(\mathrm{t}, \mathrm{J}=8 \mathrm{~Hz}, 1 \mathrm{H}), 6.92(\mathrm{~d}, \mathrm{~J}=8 \mathrm{~Hz}, 1 \mathrm{H}), 5.19(\mathrm{~s}, 1 \mathrm{H}), 3.87(\mathrm{~s}, 3 \mathrm{H}), 3.73-3.70(\mathrm{~m}, 4 \mathrm{H})$, 2.75-2.62 (m, 4H).
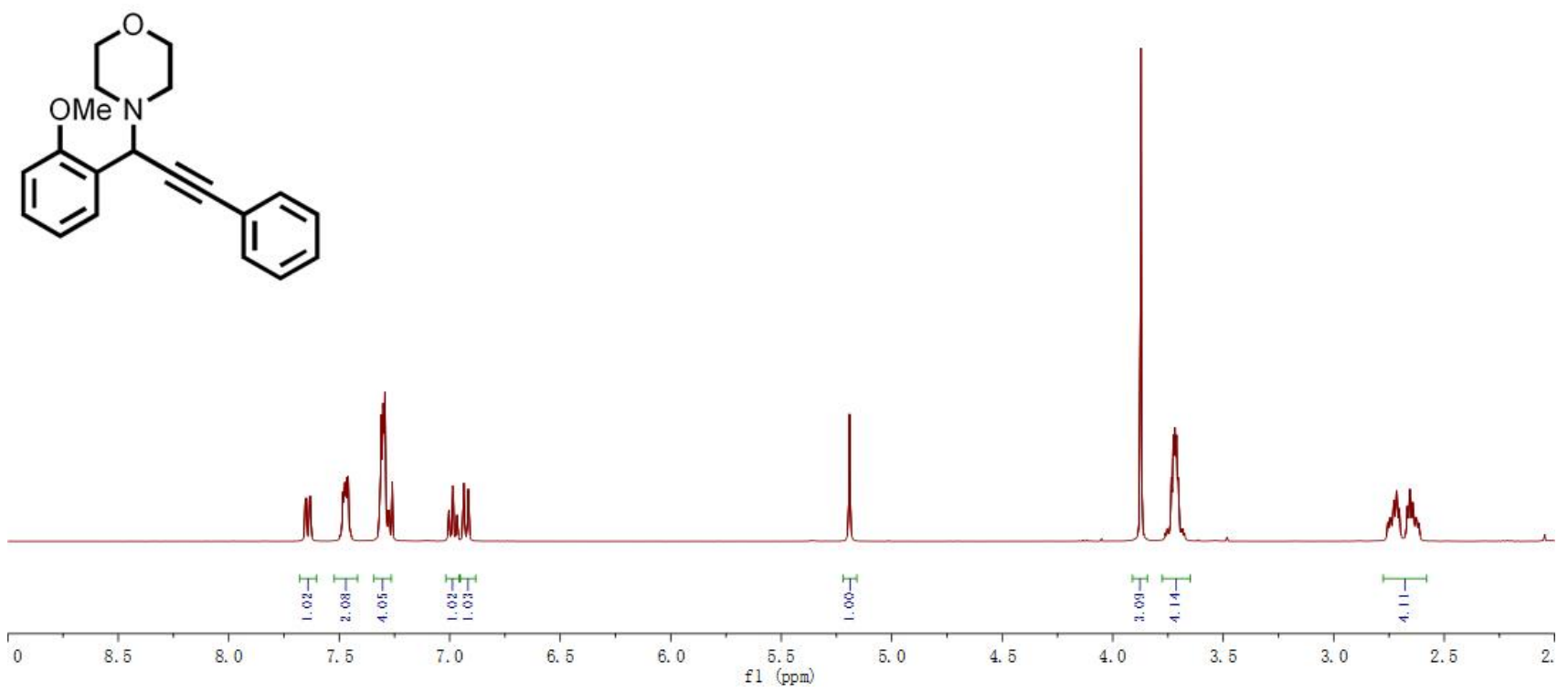

4-(3-phenyl-1-(o-tolyl)prop-2-yn-1-yl)morpholine 
${ }^{1} \mathrm{H}$ NMR $\left(400 \mathrm{MHz}, \mathrm{CDCl}_{3}\right) \delta$ 7.70-7.68 (m, 1H), 7.53-7.50 (m, 2H), 7.35-7.3 (m, 3H), 7.22-7.19 $(\mathrm{m}, 3 \mathrm{H}), 4.88(\mathrm{~s}, 1 \mathrm{H}), 3.71-3.67(\mathrm{~m}, 4 \mathrm{H}), 2.65-2.62(\mathrm{~m}, 4 \mathrm{H}), 2.48(\mathrm{~s}, 3 \mathrm{H})$.
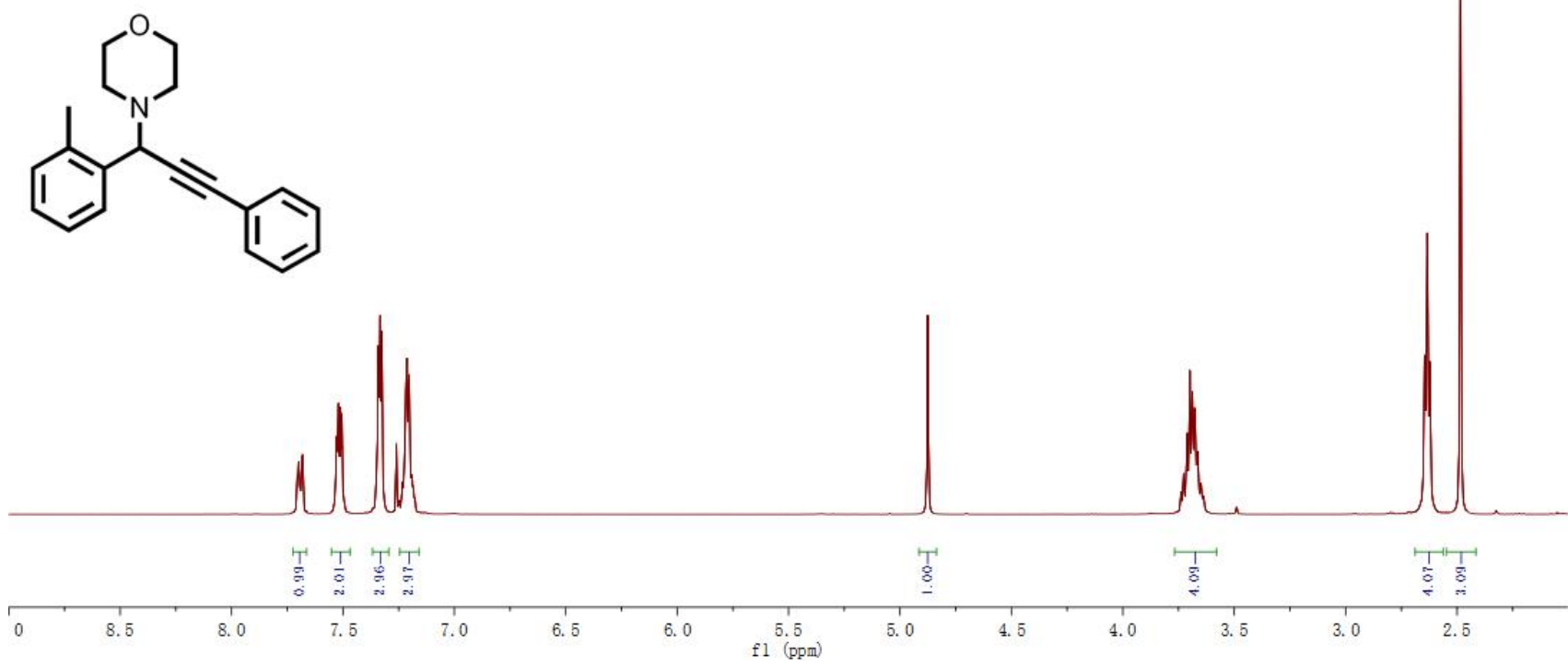

\section{4-(3-(3-fluorophenyl)-1-phenylprop-2-yn-1-yl)morpholine}

${ }^{1} \mathrm{H}$ NMR (400 MHz, $\left.\mathrm{CDCl}_{3}\right) \delta 7.62(\mathrm{~d}, \mathrm{~J}=8 \mathrm{~Hz}, 2 \mathrm{H}), 7.38(\mathrm{t}, \mathrm{J}=4 \mathrm{~Hz}, 2 \mathrm{H}), 7.33-7.28(\mathrm{~m}, 3 \mathrm{H}), 7.22$ $(\mathrm{d}, \mathrm{J}=8 \mathrm{~Hz}, 1 \mathrm{H}), 7.07-7.01(\mathrm{~m}, 1 \mathrm{H}), 4.78(\mathrm{~s}, 1 \mathrm{H}), 3.75-3.48(\mathrm{~m}, 4 \mathrm{H}), 2.64-2.61(\mathrm{~m}, 4 \mathrm{H})$.<smiles>Fc1cccc(C#CC(c2ccccc2)N2CCOCC2)c1</smiles>

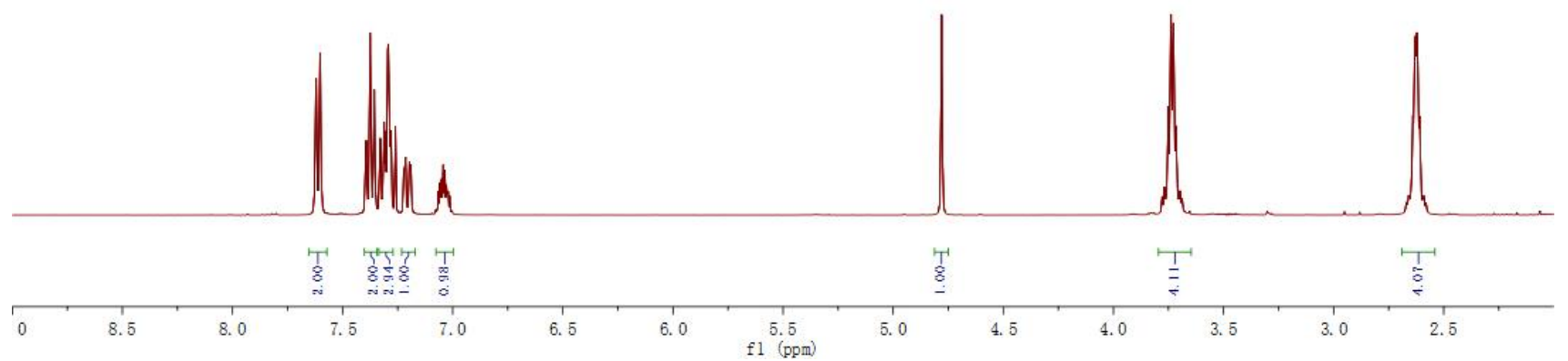

\section{4-(1-phenyl-3-(p-tolyl)prop-2-yn-1-yl)morpholine}

${ }^{1} \mathrm{H}$ NMR (400 MHz, $\left.\mathrm{CDCl}_{3}\right) \delta 7.64(\mathrm{~d}, \mathrm{~J}=8 \mathrm{~Hz}, 2 \mathrm{H}), 7.42-7.30(\mathrm{~m}, 5 \mathrm{H}), 7.15(\mathrm{~d}, \mathrm{~J}=8 \mathrm{~Hz}, 2 \mathrm{H}), 4.78$ (s, 1H), 3.75-3.71 (m, 4H), 2.64-2.62 (m, 4H), $2.36(\mathrm{~s}, 3 \mathrm{H})$. 


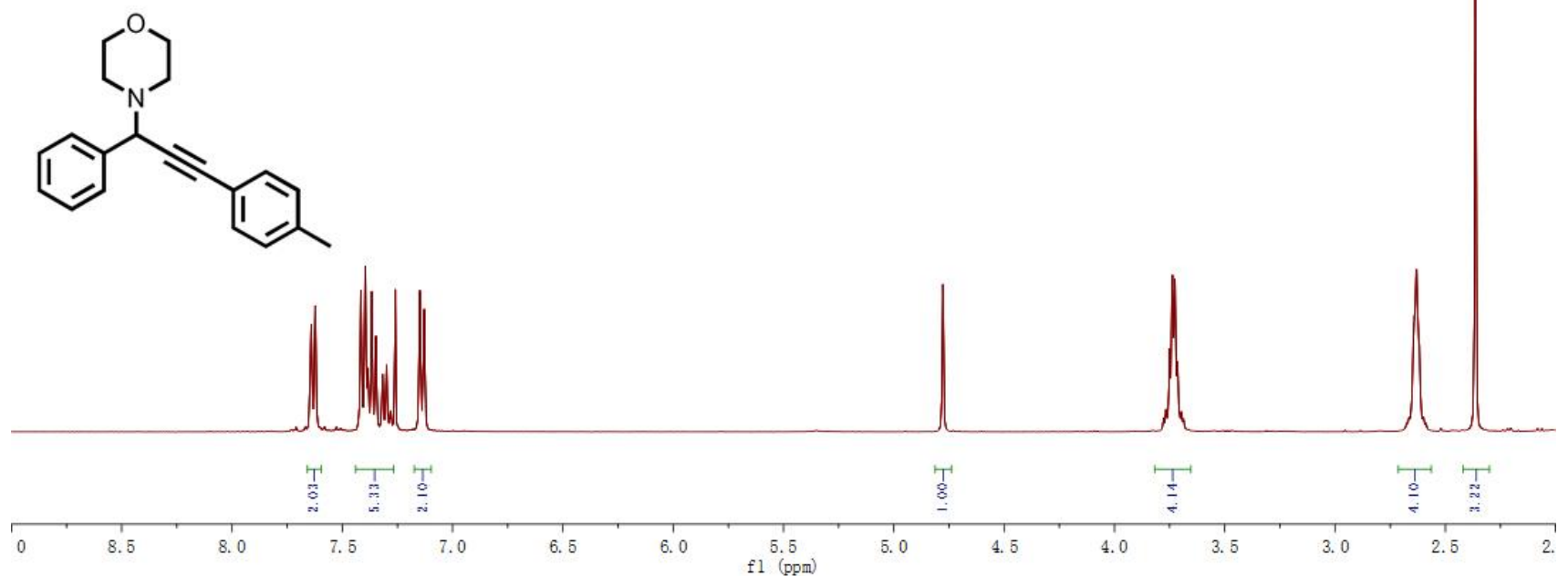

4-(3-(4-bromophenyl)-1-phenylprop-2-yn-1-yl)morpholine

${ }^{1} \mathrm{H}$ NMR (400 MHz, $\left.\mathrm{CDCl}_{3}\right) \delta 7.62(\mathrm{~d}, \mathrm{~J}=8 \mathrm{~Hz}, 2 \mathrm{H}), 7.38(\mathrm{t}, \mathrm{J}=4 \mathrm{~Hz}, 2 \mathrm{H}), 7.33-7.28(\mathrm{~m}, 3 \mathrm{H}), 7.22$ $(\mathrm{d}, \mathrm{J}=8 \mathrm{~Hz}, 1 \mathrm{H}), 7.07-7.01(\mathrm{~m}, 1 \mathrm{H}), 4.78(\mathrm{~s}, 1 \mathrm{H}), 3.75-3.48(\mathrm{~m}, 4 \mathrm{H}), 2.64-2.61(\mathrm{~m}, 4 \mathrm{H})$.

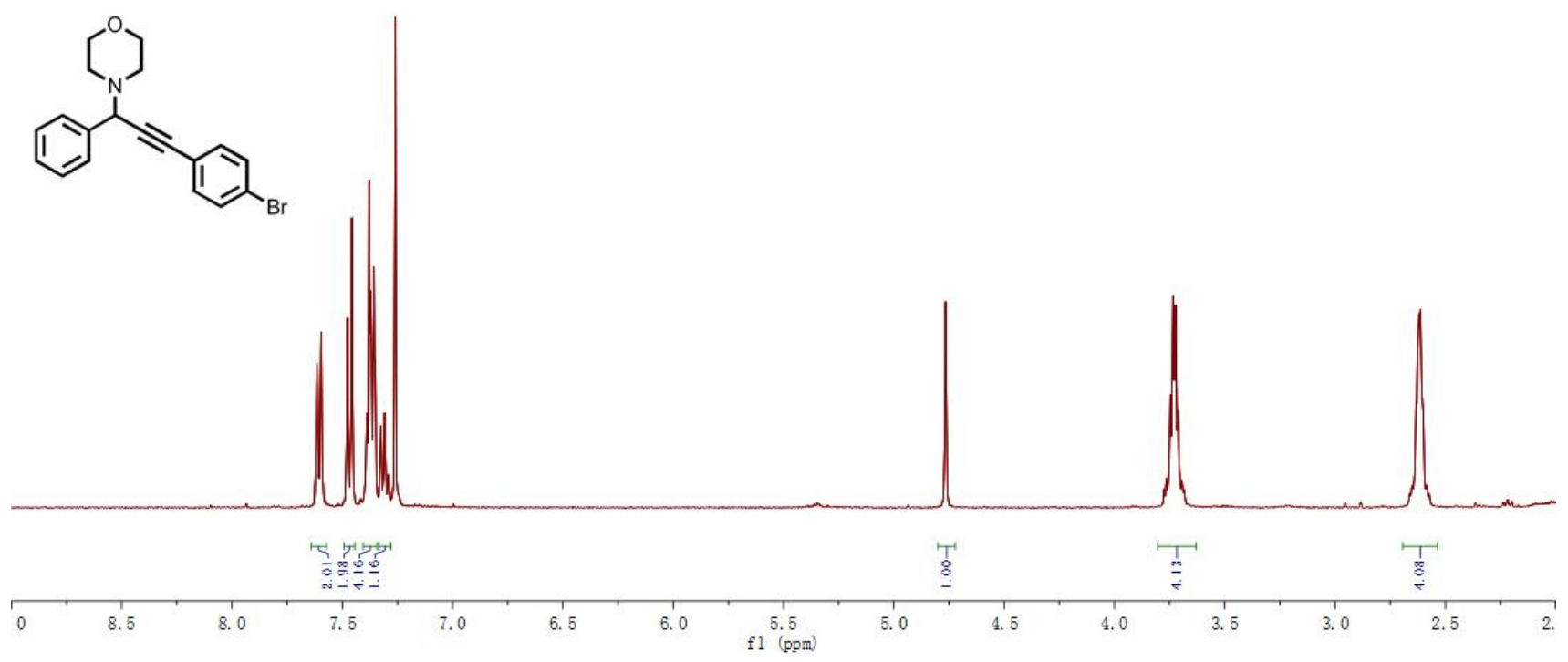

\section{4-(1-phenyl-3-(m-tolyl)prop-2-yn-1-yl)morpholine}

${ }^{1} \mathrm{H}$ NMR $\left(400 \mathrm{MHz}, \mathrm{CDCl}_{3}\right) \delta 7.65(\mathrm{~d}, \mathrm{~J}=8 \mathrm{~Hz}, 2 \mathrm{H}), 7.38-7.30(\mathrm{~m}, 5 \mathrm{H}), 7.22(\mathrm{t}, \mathrm{J}=8 \mathrm{~Hz}, 1 \mathrm{H}), 7.14$ $(\mathrm{d}, \mathrm{J}=8 \mathrm{~Hz}, 1 \mathrm{H}), 4.79(\mathrm{~s}, 1 \mathrm{H}), 3.76-3.72(\mathrm{~m}, 4 \mathrm{H}), 2.65-2.63(\mathrm{~m}, 4 \mathrm{H}), 2.35(\mathrm{~s}, 3 \mathrm{H})$. 
<smiles>O=C(O)C1CCCC1</smiles>

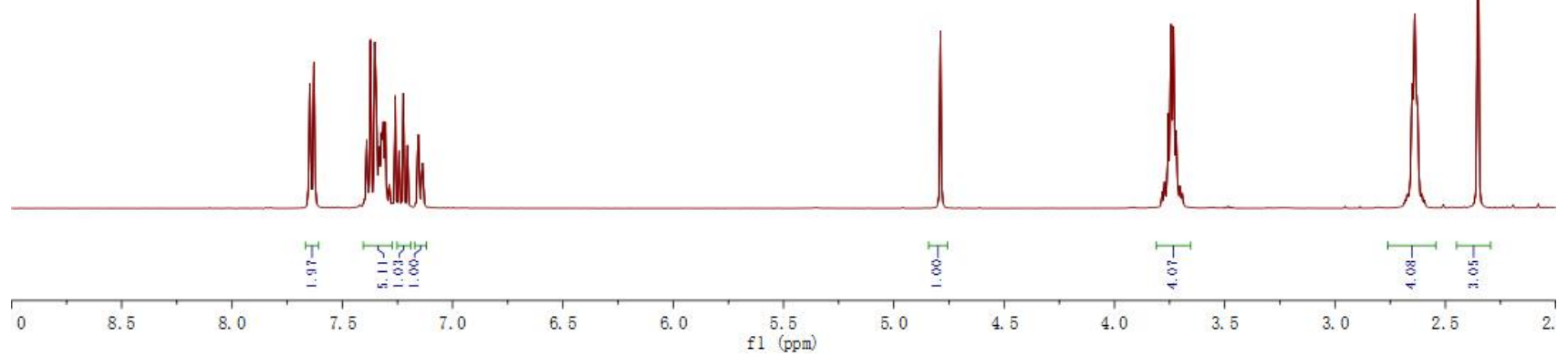

\section{4-(3-(naphthalen-2-yl)-1-phenylprop-2-yn-1-yl)morpholine}

${ }^{1} \mathrm{H}$ NMR $\left(400 \mathrm{MHz}, \mathrm{CDCl}_{3}\right) \delta 8.05(\mathrm{~s}, 1 \mathrm{H}), 7.84-7.80(\mathrm{~m}, 3 \mathrm{H}), 7.68(\mathrm{~d}, \mathrm{~J}=8 \mathrm{~Hz}, 2 \mathrm{H}), 7.58(\mathrm{dd}, \mathrm{J}=8$ $\mathrm{Hz}, 1.2 \mathrm{~Hz}, 1 \mathrm{H}), 7.53-7.48(\mathrm{~m}, 2 \mathrm{H}), 7.40$ (t, J = 8 Hz, 2H), 7.33 (t, J = 8 Hz, 1H), 4.85 (s, 1H), 3.79$3.75(\mathrm{~m}, 4 \mathrm{H}), 2.71-2.68(\mathrm{~m}, 4 \mathrm{H})$.

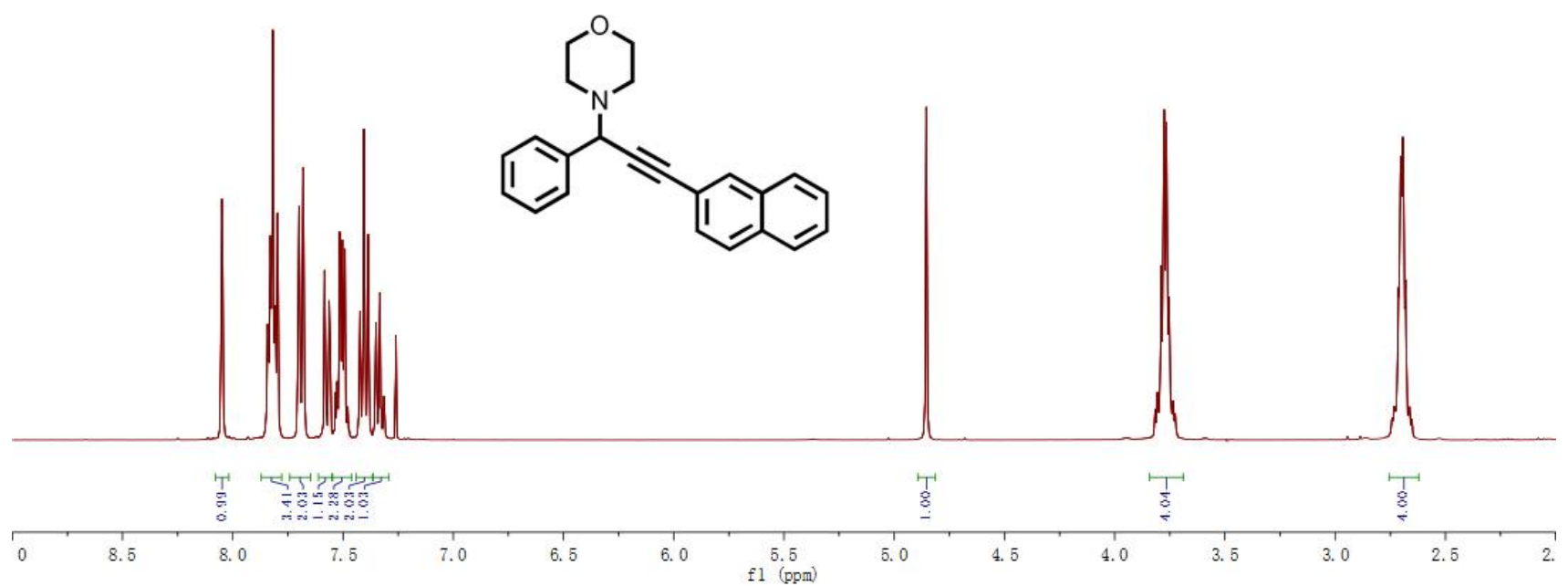

\section{4-(3-(2-methoxyphenyl)-1-phenylprop-2-yn-1-yl)morpholine}

${ }^{1} \mathrm{H}$ NMR $\left(400 \mathrm{MHz}, \mathrm{CDCl}_{3}\right) \delta 7.69(\mathrm{~d}, \mathrm{~J}=8 \mathrm{~Hz}, 2 \mathrm{H}), 7.47(\mathrm{dd}, \mathrm{J}=8 \mathrm{~Hz}, 1.2 \mathrm{~Hz}, 1 \mathrm{H}), 7.38$ (t, J = 8 $\mathrm{Hz}, 2 \mathrm{H}), 7.33-7.28(\mathrm{~m}, 1 \mathrm{H}), 6.91(\mathrm{~m}, 2 \mathrm{H}), 4.86(\mathrm{~s}, 1 \mathrm{H}), 3.91(\mathrm{~s}, 3 \mathrm{H}), 3.76-3.72(\mathrm{~m}, 4 \mathrm{H}), 2.67-2.66$ $(\mathrm{m}, 4 \mathrm{H})$. 


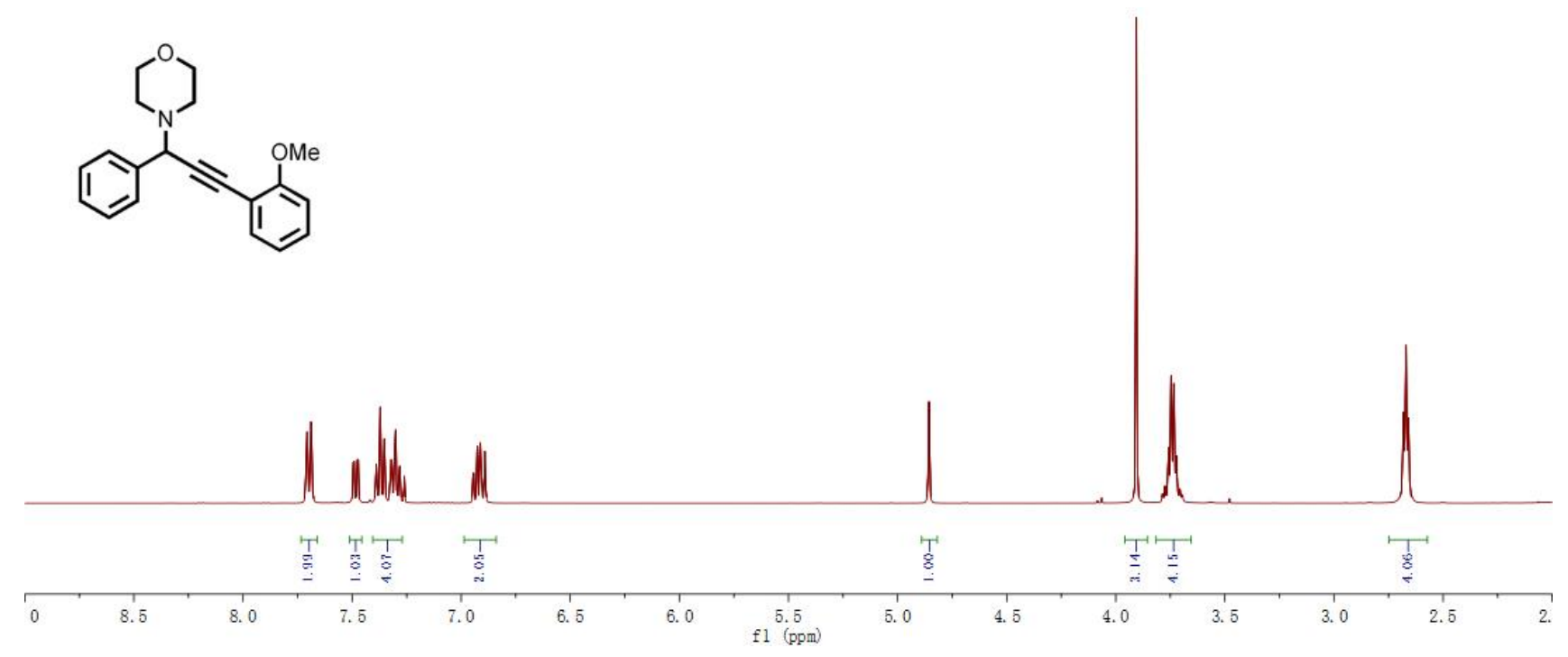

4-(3-(3-methoxyphenyl)-1-phenylprop-2-yn-1-yl)morpholine

${ }^{1} \mathrm{H} \mathrm{NMR}\left(400 \mathrm{MHz}, \mathrm{CDCl}_{3}\right) \delta 7.64(\mathrm{~d}, \mathrm{~J}=8 \mathrm{~Hz}, 2 \mathrm{H}), 7.37(\mathrm{t}, \mathrm{J}=8 \mathrm{~Hz}, 2 \mathrm{H}), 7.31(\mathrm{~d} \mathrm{~J}=8 \mathrm{~Hz}, 1 \mathrm{H})$, $7.24(\mathrm{t}, \mathrm{J}=8 \mathrm{~Hz}, 1 \mathrm{H}), 7.11(\mathrm{~d}, \mathrm{~J}=8 \mathrm{~Hz}, 1 \mathrm{H}), 7.03-7.04(\mathrm{~m}, 1 \mathrm{H}), 6.89(\mathrm{dd}, \mathrm{J}=8 \mathrm{~Hz}, 1.2 \mathrm{~Hz}, 1 \mathrm{H})$, $4.79(\mathrm{~s}, 1 \mathrm{H}), 3.82(\mathrm{~s}, 3 \mathrm{H}), 3.75-3.72(\mathrm{~m}, 4 \mathrm{H}), 2.65-2.62(\mathrm{~m}, 4 \mathrm{H})$.

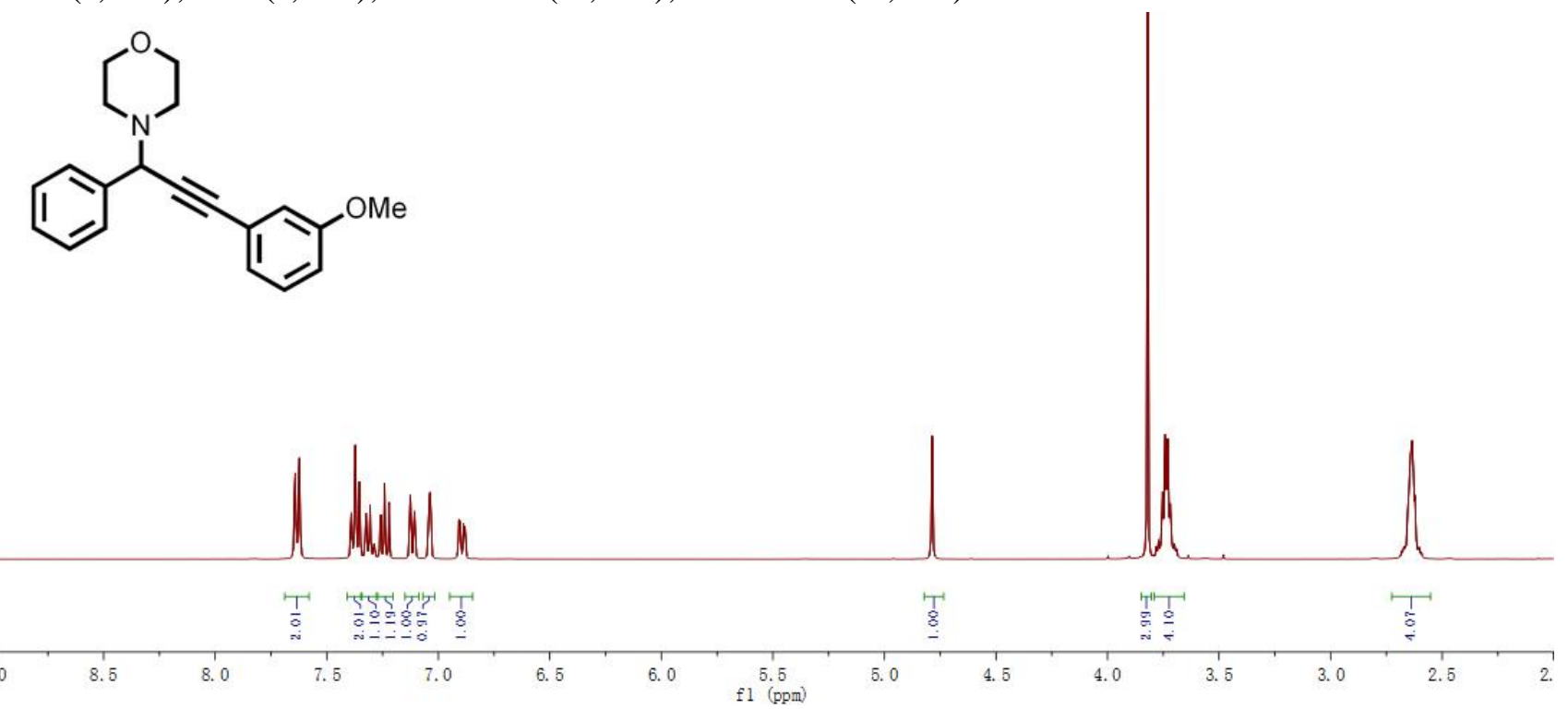

4-(3-(4-methoxyphenyl)-1-phenylprop-2-yn-1-yl)morpholine

${ }^{1} \mathrm{H}$ NMR $\left(400 \mathrm{MHz}, \mathrm{CDCl}_{3}\right) \delta 7.65(\mathrm{~d}, \mathrm{~J}=8 \mathrm{~Hz}, 2 \mathrm{H}), 7.46(\mathrm{~d}, \mathrm{~J}=8 \mathrm{~Hz}, 2 \mathrm{H}), 7.37(\mathrm{t}, \mathrm{J}=8 \mathrm{~Hz}, 2 \mathrm{H})$, $7.32(\mathrm{t}, \mathrm{J}=8 \mathrm{~Hz}, 1 \mathrm{H}), 6.87(\mathrm{~d}, \mathrm{~J}=8 \mathrm{~Hz}, 2 \mathrm{H}), 4.77(\mathrm{~s}, 1 \mathrm{H}), 3.82(\mathrm{~s}, 3 \mathrm{H}), 3.75-3.72(\mathrm{~m}, 4 \mathrm{H}), 2.65-2.62$ $(\mathrm{m}, 4 \mathrm{H})$. 

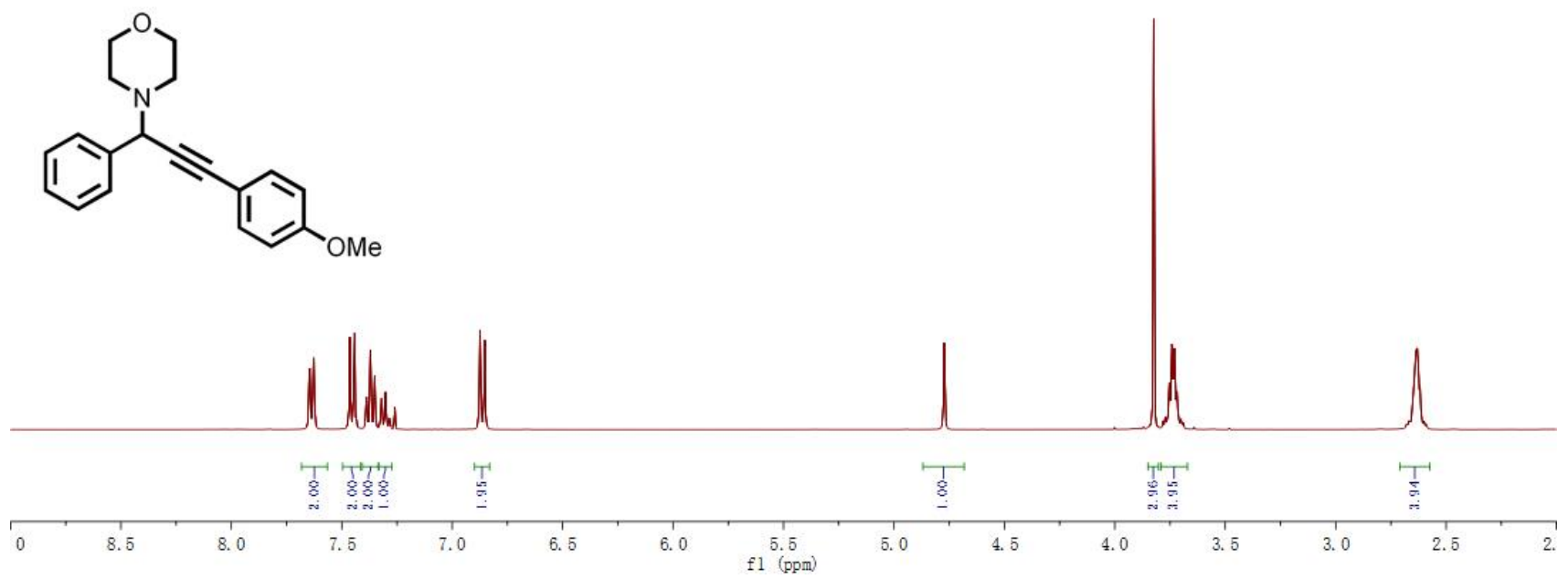

\section{4-(3-(naphthalen-2-yl)-1-(pyren-1-yl)prop-2-yn-1-yl)morpholine}

${ }^{1} \mathrm{H}$ NMR $\left(400 \mathrm{MHz}, \mathrm{CDCl}_{3}\right) \delta 8.66(\mathrm{~d}, \mathrm{~J}=8 \mathrm{~Hz}, 1 \mathrm{H}), 8.50(\mathrm{~d}, \mathrm{~J}=8 \mathrm{~Hz}, 1 \mathrm{H}), 8.17-8.20(\mathrm{~m}, 4 \mathrm{H}), 8.10$ $(\mathrm{s}, 1 \mathrm{H}), 8.08(\mathrm{~s}, 2 \mathrm{H}), 8.03(\mathrm{t}, \mathrm{J}=8 \mathrm{~Hz}, 1 \mathrm{H}), 7.82-7.84(\mathrm{~m}, 3 \mathrm{H}), 7.61(\mathrm{~d}, \mathrm{~J}=8 \mathrm{~Hz}, 1 \mathrm{H}), 7.50-7.52$ $(\mathrm{m}, 2 \mathrm{H}), 5.76(\mathrm{~s}, 1 \mathrm{H}), 3.70-3.78(\mathrm{~m}, 4 \mathrm{H}), 2.79-2.85(\mathrm{~m}, 4 \mathrm{H})$.

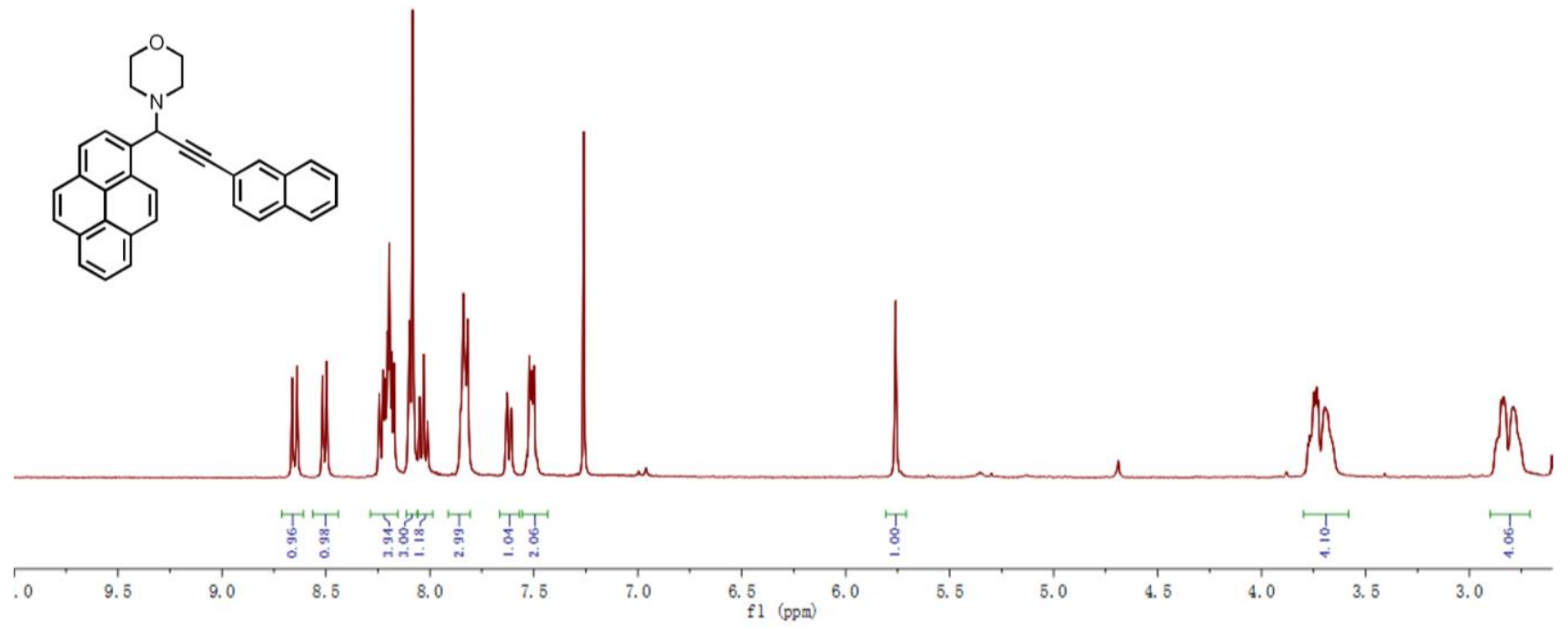

4-(1-(4-(anthracen-9-yl)phenyl)-3-(naphthalen-2-yl)prop-2-yn-1-yl)morpholine

${ }^{1} \mathrm{H} \mathrm{NMR}\left(400 \mathrm{MHz}, \mathrm{CDCl}_{3}\right) \delta 8.51(\mathrm{~s}, 1 \mathrm{H}), 8.11(\mathrm{~s}, 1 \mathrm{H}), 8.05(\mathrm{~d}, \mathrm{~J}=8 \mathrm{~Hz}, 2 \mathrm{H}), 7.89$ (dd, J = $8 \mathrm{~Hz}$, 2H), 7.82-7.85 (m, 3H), 7.86 (d, J = 8 Hz, 2H), 7.61 (dd, J = 8 Hz, 2.4 Hz, 1H), 7.45-7.52 (m, 6H), 7.34-7.38 (td, J = $8 \mathrm{~Hz}, 1.2 \mathrm{~Hz}, 2 \mathrm{H}), 5.04$ (s, 1H), 3.85 (m, 4H), 2.80 (m, 4H). 


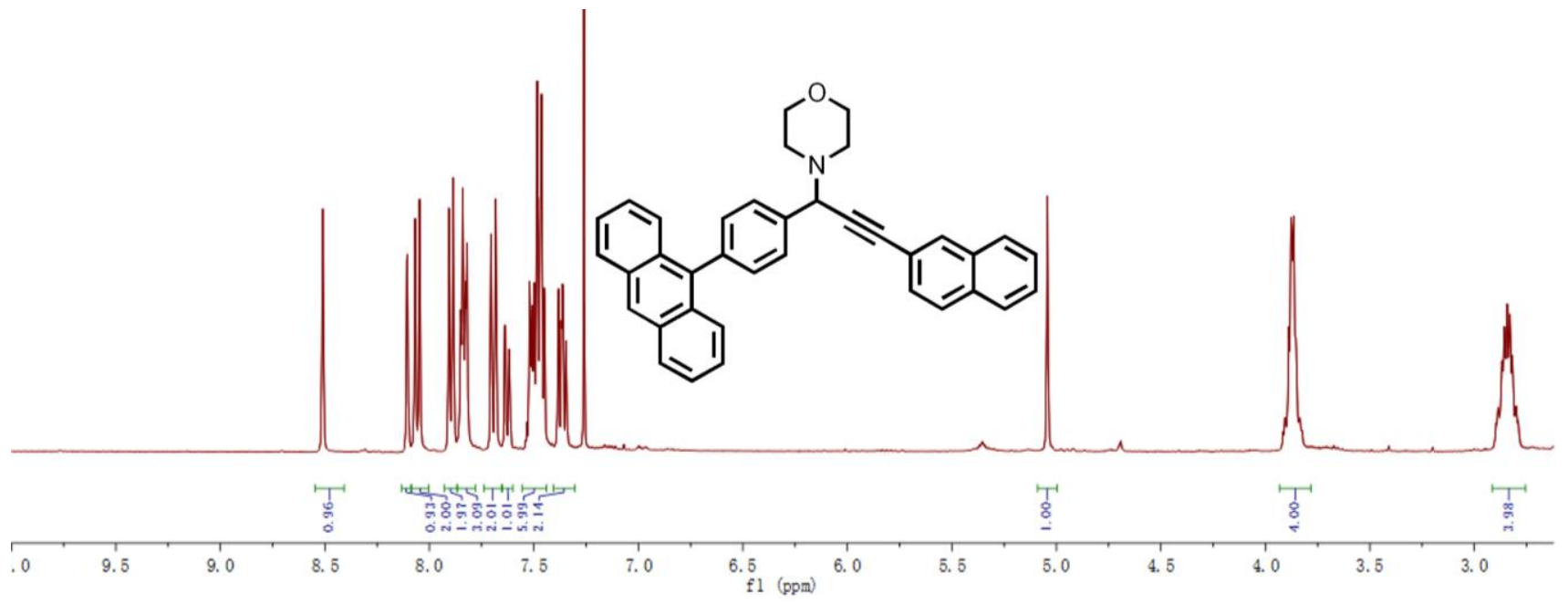

4-(1-(3,5-bis(benzyloxy)phenyl)-3-(naphthalen-2-yl)prop-2-yn-1-yl)morpholine ${ }^{1} \mathrm{H}$ NMR (400 MHz, $\left.\mathrm{CDCl}_{3}\right) \delta 8.01(\mathrm{~s}, 1 \mathrm{H}), 7.79-7.84(\mathrm{~m}, 3 \mathrm{H}), 7.49-7.54(\mathrm{~m}, 3 \mathrm{H}), 7.43-7.45(\mathrm{~m}$, $4 \mathrm{H}), 7.30-7.39(\mathrm{~m}, 6 \mathrm{H}), 6.95(\mathrm{~d}, \mathrm{~J}=2.4 \mathrm{~Hz}, 2 \mathrm{H}), 6.95(\mathrm{t}, \mathrm{J}=1.2 \mathrm{~Hz}, 1 \mathrm{H}), 5.08(\mathrm{~s}, 4 \mathrm{H}), 4.75(\mathrm{~s}, 1 \mathrm{H})$, 3.71-3.75 (m, 4H), 2.62-2.67 (m, 4H).

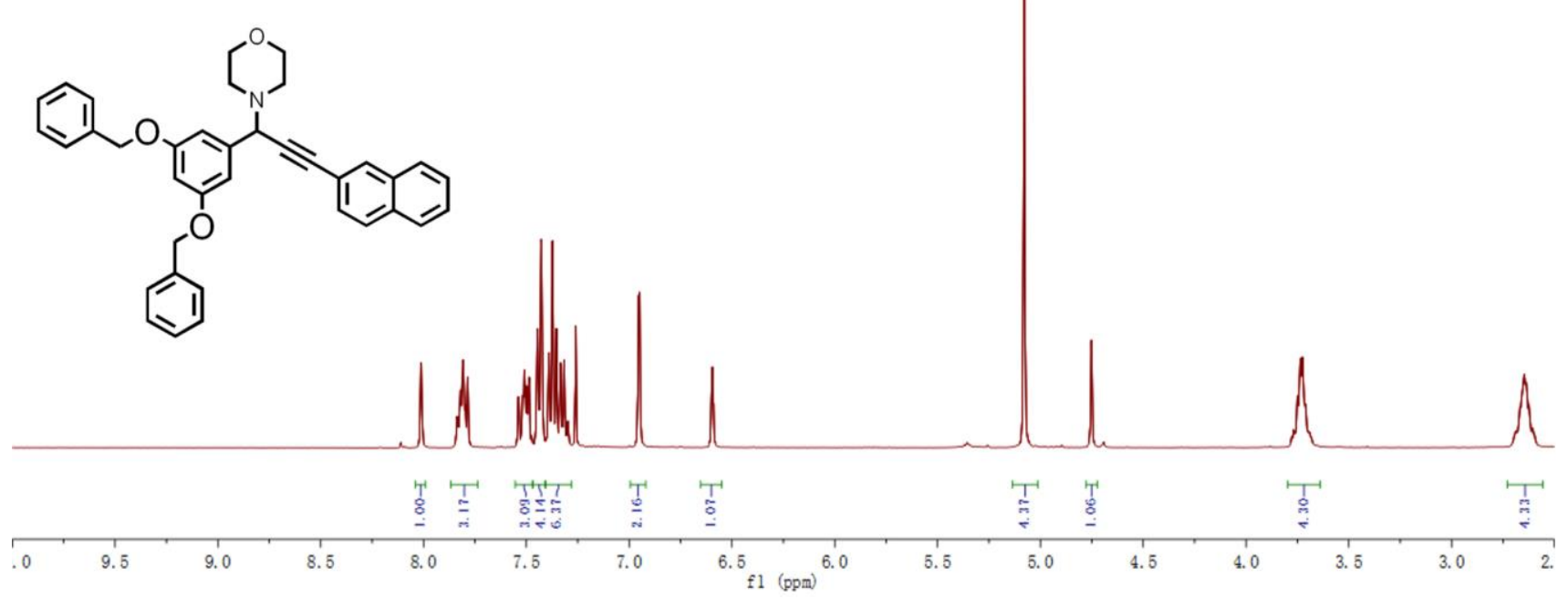

4-(1-(3,5-bis((3,5-di-tert-butylbenzyl)oxy)phenyl)-3-(naphthalen-2-yl)prop-2-yn-1-yl)morpholine

${ }^{1} \mathrm{H}$ NMR $\left(400 \mathrm{MHz} \mathrm{CDCl}_{3}\right) \delta 8.03(\mathrm{~s}, 1 \mathrm{H}), 7.78-7.83(\mathrm{~m}, 3 \mathrm{H}), 7.54(\mathrm{dd}, \mathrm{J}=8 \mathrm{~Hz}, 2.4 \mathrm{~Hz}, 1 \mathrm{H}), 7.48-$ $7.51(\mathrm{~m}, 2 \mathrm{H}), 7.42(\mathrm{t}, \mathrm{J}=4 \mathrm{~Hz}, 2 \mathrm{H}), 7.30(\mathrm{~d}, \mathrm{~J}=2.4 \mathrm{~Hz}, 4 \mathrm{H}), 7.0(\mathrm{~d}, \mathrm{~J}=2.4 \mathrm{~Hz}, 2 \mathrm{H}), 6.67(\mathrm{t}, \mathrm{J}=2.4$ $\mathrm{Hz}, 1 \mathrm{H}), 5.06$ (s, 4H), $4.78(\mathrm{~s}, 1 \mathrm{H}), 3.71-3.75(\mathrm{~m}, 4 \mathrm{H}), 2.63-2.73(\mathrm{~m}, 4 \mathrm{H}), 1.34(\mathrm{~s}, 36 \mathrm{H})$. 


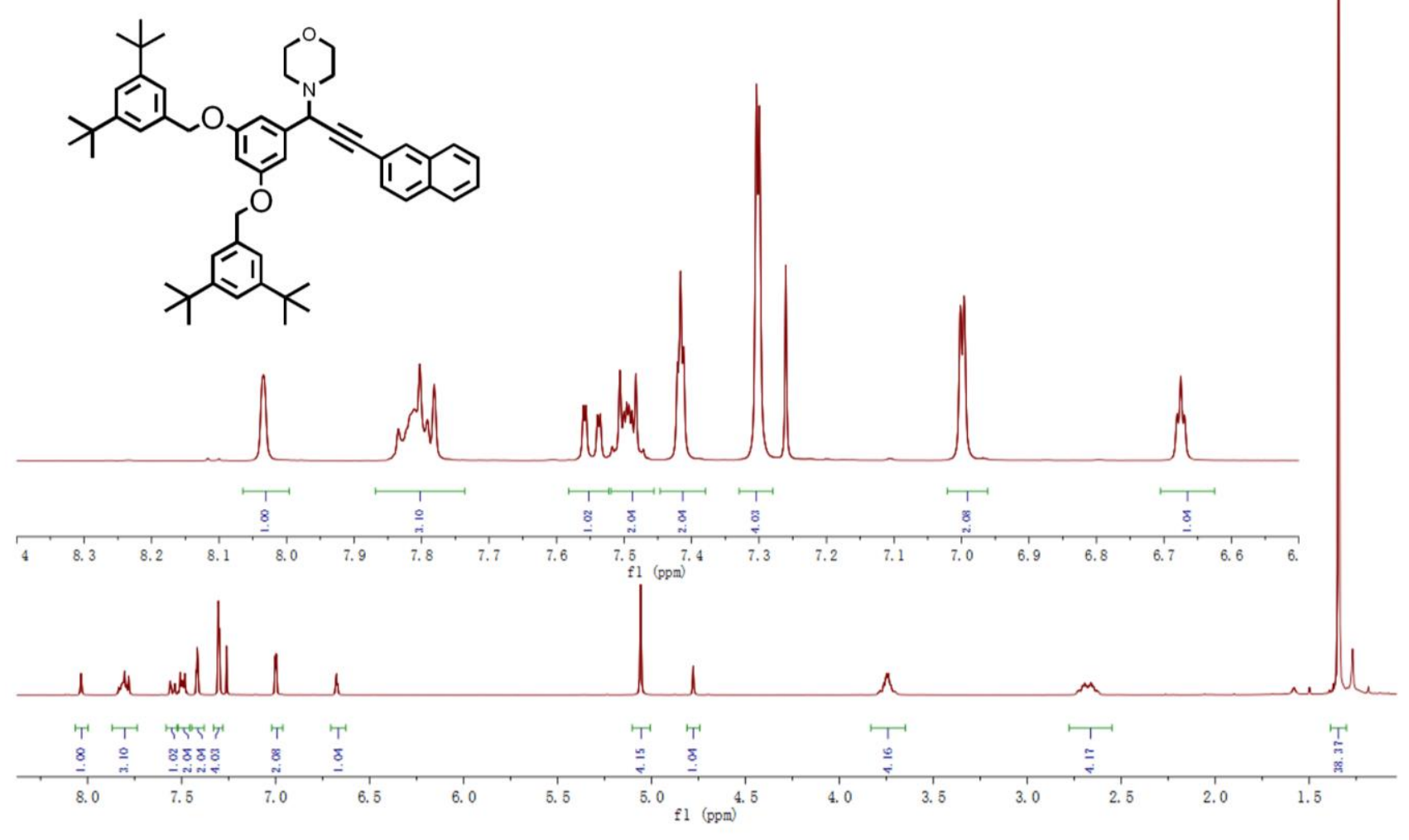

\subsection{Recycle Experiments}<smiles>CCCC[C@@H](C)OC</smiles><smiles>O=C1NC(c2ccc(Cl)cc2)Nc2ccccc21</smiles>

\begin{tabular}{|l|l|l|l|l|l|l|}
\hline \multicolumn{2}{|l|}{ Run } & 1 & 2 & 3 & 4 & 5 \\
\hline $\begin{array}{l}\text { Conversion } \\
(\%)\end{array}$ & LMOF 1 & 87 & 88 & 80 & 85 & 70 \\
\cline { 2 - 7 } & MON 1 & 99 & 99 & 96 & 84 & 77 \\
\hline
\end{tabular}

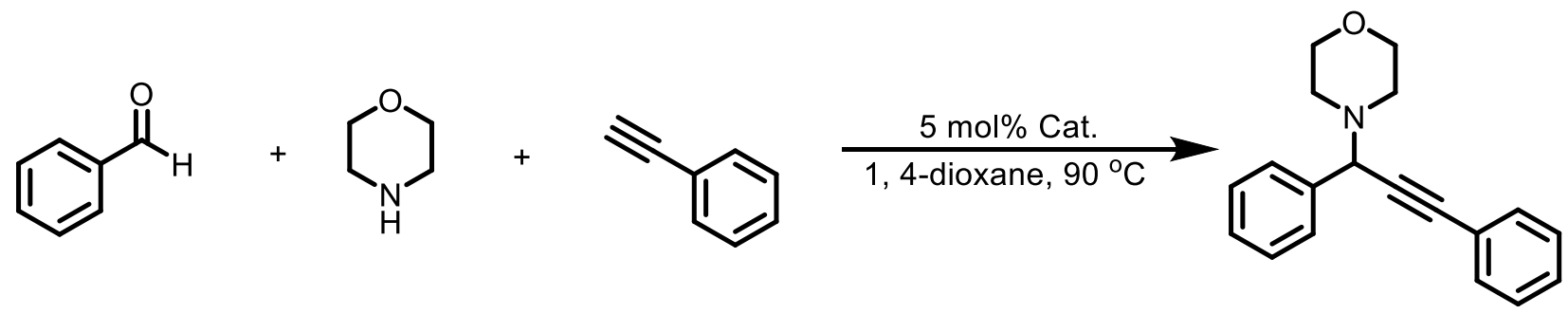

\begin{tabular}{|l|l|l|l|l|l|l|}
\hline \multicolumn{2}{|l|}{ Run } & 1 & 2 & 3 & 4 & 5 \\
\hline $\begin{array}{l}\text { Conversion } \\
(\%)\end{array}$ & LMOF 1 & 87 & 87 & 79 & 74 & 74 \\
\cline { 2 - 7 } & MON 1 & 92 & 93 & 85 & 76 & 77 \\
\hline
\end{tabular}


19. Figure S16. FT-IR spectra of LMOF 1 and MON 1 after 5 catalytic runs

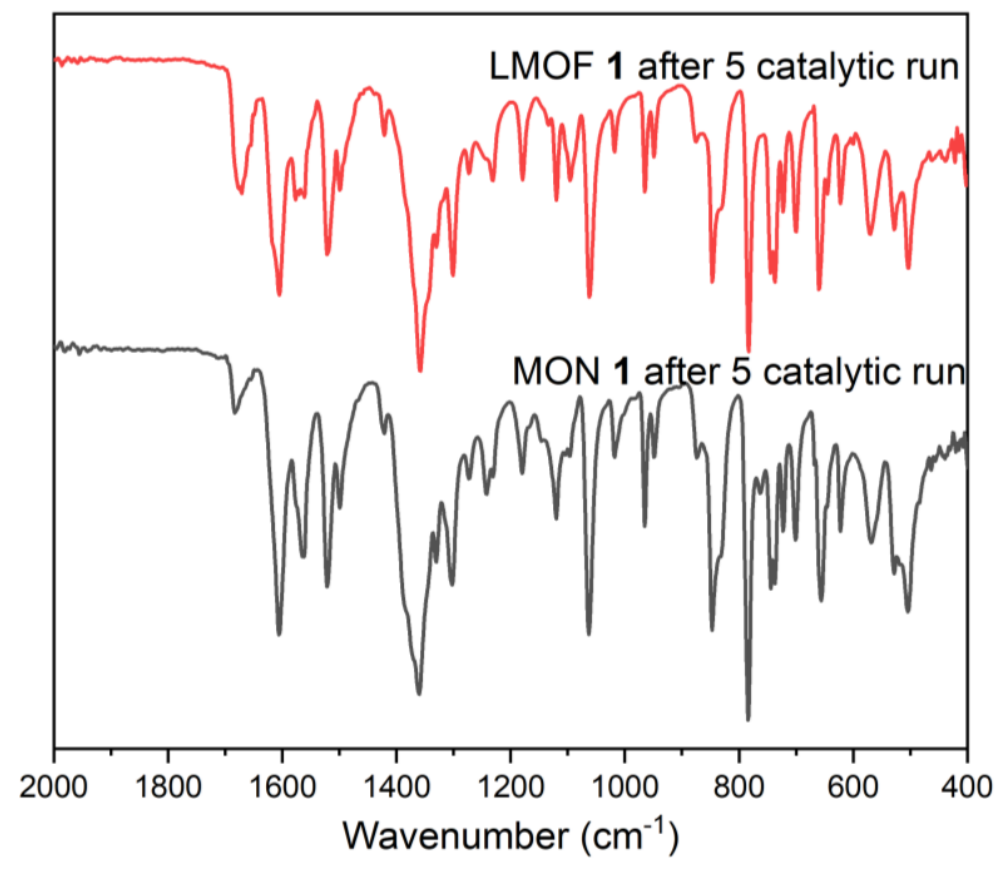

